Florida International University FIU Digital Commons

3-21-2017

\title{
Deposition of Copper Nanoparticles on 2D Graphene NanoPlatelets via Cementation Process
}

Luiza Da Fontoura

Florida International University, 1font025@fiu.edu

DOI: $10.25148 /$ etd.FIDC001818

Follow this and additional works at: https://digitalcommons.fiu.edu/etd

Part of the Other Materials Science and Engineering Commons

\section{Recommended Citation}

Da Fontoura, Luiza, "Deposition of Copper Nanoparticles on 2D Graphene NanoPlatelets via Cementation Process" (2017). FIU Electronic Theses and Dissertations. 3164.

https://digitalcommons.fiu.edu/etd/3164 


\section{FLORIDA INTERNATIONAL UNIVERSITY}

Miami, Florida

\section{DEPOSITION OF COPPER NANOPARTICLES ON 2D GRAPHENE \\ NANOPLATELETS VIA CEMENTATION PROCESS}

A thesis submitted in partial fulfillment of

the requirements for the degree of

MASTER OF SCIENCE

in

MATERIALS SCIENCE AND ENGINEERING

by

Luiza Da Fontoura 
To: Interim Dean Ranu Jung

College of Engineering and Computing

This thesis, written by Luiza Da Fontoura, and entitled Deposition of Copper Nanoparticles on 2D Graphene Nanoplatelets via Cementation Process, having been approved in respect to style and intellectual content, is referred to you for judgment.

We have read this thesis and recommend that it be approved.

Chunlei Wang

Benjamin Boes1, Co-Major Professor

Arvind Agarwal, Co-Major Professor

Date of Defense: March 21, 2017

The thesis of Luiza Da Fontoura is approved.

Interim Dean Ranu Jung

College of Engineering and Computing

Andrés G. Gil

Vice President for Research and Economic Development and Dean of the University Graduate School

Florida International University, 2017 


\section{DEDICATION}

I dedicate this thesis to my parents and my brother. The love I have for them is my motivation in all aspects of my life. 


\section{ACKNOWLEDGMENTS}

I would like to thank all that have aided and guided me in the progress of this thesis. I'd like to sincerely thank my advisors, Dr. Arvind Agarwal and Dr. Benjamin Boesl for their continued support and guidance. Thank you for giving me the opportunity to work with you and be part of the Plasma Forming Lab, this experience has been priceless.

I extend my thanks to all PFL members, working with you all has been extremely rewarding and memorable. Special thanks to Archana Loganathan, for her continued guidance, support, and encouragement. I am very happy and grateful to have met, worked, and gotten to know you. I would also like to thank Dr. Cheng Zhang, Dr. Chris Rudolf, and Sara Renfigo for their assistance and guidance. I am very grateful to the AMERI staff, in particular Dr. Alex Franco, Dr. Shravana Katakam, and Patrick Roman. I would also like to thank my committee member Dr. Chunlei Wang for her helpful suggestions. I'm grateful to Tiziana Leoni for her helpfulness and kindness.

Most importantly, I'd like to thank my family for their continued love and support. Eu amo muito vocês. I am thankful to my mother for her continued love and to my father, for teaching me to always strive for more. I wish to thank my grandmother vó Vanilde, for showing me what true strength is, and my aunt, tia Tereza and cousin Renata, both of whom I love and can always count on. I'm thankful for my little brothers Christopher and Dudu's love and admiration, which inspires me to work harder. I'm grateful to my friend Rebecca June Kralewski and the June family (Bobby, Kim and Dan) for their continued love and support. I'm grateful to Chris and Dawn, as well as Ali, for her energetic support throughout this thesis. Finally, I'd like to thank the William's and Young's for welcoming my mother and I into their families with love. 


\title{
ABSTRACT OF THE THESIS \\ DEPOSITION OF COPPER NANOPARTICLES ON 2D GRAPHENE \\ NANOPLATELETS VIA CEMENTATION PROCESS
}

\author{
by \\ Luiza Da Fontoura \\ Florida International University, 2017 \\ Miami, Florida

\begin{abstract}
Professor Arvind Agarwal, Co-Major Professor
Professor Benjamin Boesl, Co-Major Professor
\end{abstract}

The main goal of this thesis is to deposit metal particles on the surface of 2D nanoplatelets using a controlled cementation process. As a proof of concept, copper $(\mathrm{Cu})$ and Graphene Nanoplatelets (GNP) were chosen as the representative metal and 2D nanoplatelets, respectively. Specific goals of this study include depositing nanometer scale $\mathrm{Cu}$ particles on the surface of GNP at a low concentration (approximately 5 vol.\%) while maintaining clustering and impurities at a minimum. Parametric studies were done to attain these goals by investigating various metallic reducer types and morphologies, GNP surface activation process, acid volume $\%$ and copper (II) sulfate concentrations. Optimal conditions were obtained with $\mathrm{Mg}$ ribbon as a reducer, 3 minutes of activation, 1 vol.\% of acetic acid and $0.01 \mathrm{M} \mathrm{CuSO}_{4}$. The GNP-Cu powder synthesized in this work is a precursor material to be consolidated via spark plasma sintering (SPS) to make a nacre-like, layered structure for future studies. 


\section{TABLE OF CONTENTS}

CHAPTER

PAGE

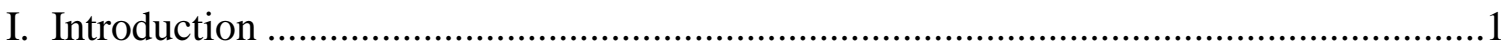

1.1. Significance of this Research.......................................................................

1.1.2. Utilizing the High Surface Area of 2D Materials as an Asset ...........1

1.1.2.1. Graphene NanoPlatelets (GNP) ......................................2

1.1.2. Cementation Reaction Process............................................................ 3

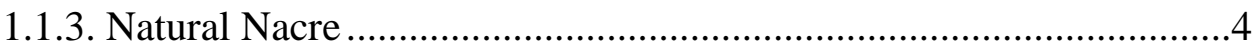

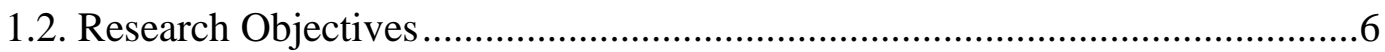

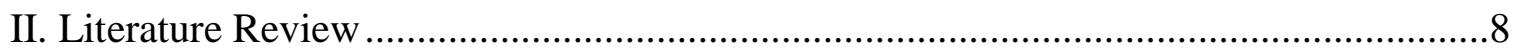

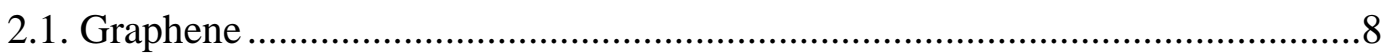

2.2. Nanoparticle (NP) Metallic Deposition on 2D Materials ...............................12

2.2.1. Chemical Reduction Techniques ....................................................13

2.2.2. Electrochemical Techniques .......................................................15

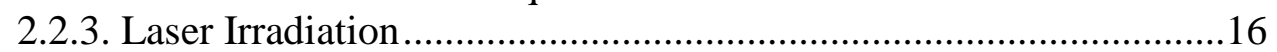

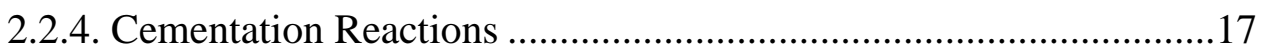

2.3. Artificial Nacre ................................................................................... 18

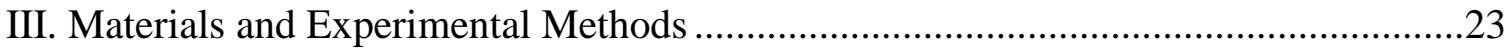

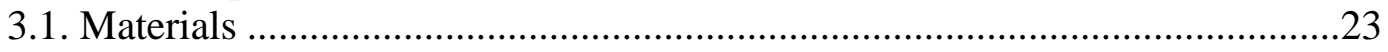

3.1.1. Graphene Nanoplatelets (GNP) ……………………………......23

3.1.2. Cementation Reaction Materials .....................................................24

3.2. Metallic Particle Deposition via Cementation Process.....................................24

3.2.1. Dispersion of Nanoplatelets ..........................................................24

3.2.2. Cementation Reaction Synthesis Steps ...........................................26

3.2.2.1. Preparation of Metal Reducer Surface ...............................26

3.2.2.1. Preparation of $\mathrm{CuSO}_{4}$ Salt Solution and Surface Activation of GNP ........................................................22

3.2.2.3. Start of Reaction via Introduction of Reducer ..................28

3.2.2.4. Filtering and Washing .....................................................28

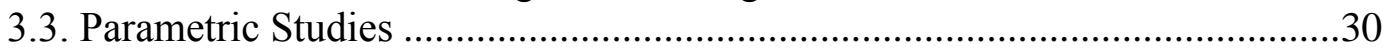

3.4. Characterization of GNP-Cu Powder................................................................

3.4.1. Microstructural Characterization and Chemical Composition .........32

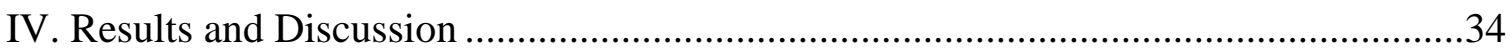

4.1. Influence of Input Parameters on Outcome ……….......................................34

4.1.1. Influence of Metallic Reducer ........................................................35

4.1.1.1. Type: Determining Most Efficient Reducer (Mg vs Zn) .36

4.1.1.2. Form: Determining Most Efficient Form of Mg ...............39

4.1.1.3. Concentration: Determining Most Efficient

Concentration of $\mathrm{Mg}$ as Reducer

4.1.2. Influence of Acetic Acid as Surface Activator .................................44 
4.1.2.1. GNP Surface Activation by Acetic Acid

4.1.2.1. Varying Concentration of Acetic Acid to Minimize Impurities

4.1.3. Influence of Copper(II) Sulfate.......................................................51

4.2. Preliminary Characterization of Sintered GNP-Cu Compacts........................59

4.2.1. Fracture Surface of Compacts.......................................................62

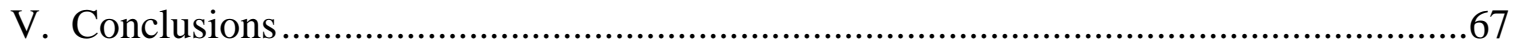

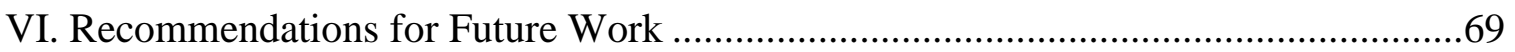

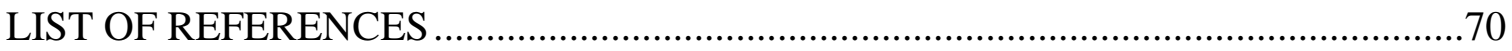




\section{LIST OF TABLES}

TABLE

PAGE

2.1. Techniques and Outcomes for Metallic Nanoparticle (NP) Deposition

2.2. Techniques employed to synthesize nacre-like structures

3.1. Parametric studies of metal reducers, acetic acid and copper(II) sulfate concentration to achieve a uniform deposition of nanometer-sized $\mathrm{Cu}$ particles on GNP with minimum impurities.

3.2. Studies of $\mathrm{Mg}$ ribbon and $\mathrm{Zn}$ pieces in order to discern most effective reducer at varying $\mathrm{CuSO}_{4}$ concentrations

3.3. Parametric studies of $\mathrm{Mg}$ ribbon concentration, acetic acid surface activation process and varying volume percentages, and $\mathrm{CuSO} 4$ concentration carried out using $\mathrm{Mg}$ ribbon as the reducer

4.1. Parametric studies carried out on metal reducer investigating type, form and concentration

4.2. Effectiveness of $\mathrm{Mg}$ Ribbon and $\mathrm{Zn}$ Pieces as reducers at varying $\mathrm{CuSO}_{4}$ concentrations.

4.3. Effectiveness of 0.66 moles of $\mathrm{Zn}$ and $\mathrm{Mg}$ as reducers to precipitate pure copper versus copper(I) oxide

4.4. Effectiveness of different forms of $\mathrm{Mg}$ as reducers in precipitating pure copper versus copper(I) oxide.

4.5. Parametric investigations of surface activation process and volume $\%$ using acetic acid with optimum conditions highlighted in blue

4.6. Varying concentrations of copper(II) sulfate solution investigated in this study .......54

4.7. Decreasing particle and crystallite size of precipitated copper particles as a function of decreasing $\mathrm{CuSO}_{4}$ concentration and acetic acid volume \%

4.8. Parametric studies of metal reducers, acetic acid and copper(II) sulfate concentration with highlighted optimum conditions to achieve a uniform deposition of nanometer-sized $\mathrm{Cu}$ particles on GNP with minimum impurities

4.9. GNP-Cu powder synthesis conditions detailing the concentrations of $\mathrm{Mg}$ ribbon and $\mathrm{CuSO}_{4}$ surface activation process length, and volume $\%$ of acetic acid used. 
4.10. Temperature, pressure, heating rate and hold time conditions used to sinter GNP-Cu compacts via Spark Plasma Sintering

4.11. GNP-Cu powder and compact densities and the calculated volume percentages of GNP and $\mathrm{Cu}$....... 


\section{LIST OF FIGURES}

FIGURE

PAGE

1.1 Schematic of Cementation Reaction. (a) Glacial acetic acid used to activate the GNP surface, represented as black ovals. (b-d) Reduction of copper ions on the GNP surface by reducer metal.

1.2 (a) Schematic of GNP (represented by grey ovals) with copper particles (represented by red cubes) deposited on its surface post cementation reaction.

(b) Schematic of GNP-Cu compact post SPS shows a layered nacre-like structure of nanometer thin copper metal amidst GNPs.

2.1 (a) HRTEM image of single and bilayer graphene [18]. (b) HRSTM image of mechanically exfoliated single-layer graphite showing honeycomb structure [19].....9

2.2. (a-c) SEM images showing varied morphologies and quantity of $\mathrm{Fe}_{3} \mathrm{O}_{4} \mathrm{NPs}$ deposited onto (a,b) BNNP and (c) GO via co-precipitation. Images (a) and (b) shows the variance in coating when the weight $\%$ of iron oxide NP is changed from (a) 30\% to (b) 100\%. Images (c) and (d) shows iron oxide:GO ratio of 1:6 and $1: 3$ respectively $[52,53]$.

2.3. $(a, b)$ SEM images showing varied morphologies of AuNPs electrodeposited onto the (a) $\mathrm{NH}_{2}$ and (b) BLG functionalized $\mathrm{rGO}$ surface via electrodeposition. [55,56] .16

2.4. (a) SEM of a cross section of natural nacre. (b) close up of same image with arrows showing the interface between $\mathrm{CaCO}_{3}$ and organic matrices. [14]

3.1. (a-b) SEM images of pure GNP powder showing its (a) top surface and (b) a cross section image showing a stack of few GNPs. (c) TEM image of pure GNP powder.

3.2. (a) Vibra-Cell VCX750 ultrasonic processor equipped with a $3 / 4$ " high gain probe.

(b) Tip-sonicating GNP in acetone medium

3.3. (a) As purchased zinc sheet cut into $2 \mathrm{~cm}$ strips. (b) As-purchased magnesium ribbon coils. (c) Magnesium strip before (top) and after polishing (bottom)

3.4. (a) Slightly blue hue of $0.01 \mathrm{M}$ copper (II) sulfate aqueous solution continuously stirred by magnetic stirrer. (b) A picture during cementation reaction. A piece of the magnesium ribbon can be observed at the bottom while the solution is continuously stirred.

3.5. (a) Magnesium ribbon reducer after the reaction ends. (b) Gravimetric filtering 
setup: filter paper is placed inside glass funnel and filtrate is released into Erlenmeyer flask. (c) Wet GNP-Cu powder inside filter paper left overnight to dry

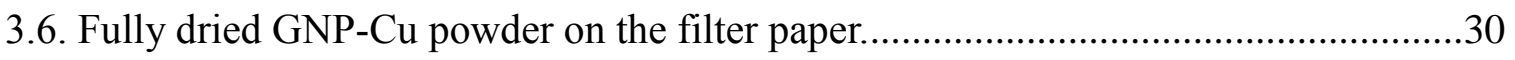

4.1. Influence of input parameters on the desired final goals of this study $\ldots . . . . . . . . . . . . . . . .35$

4.2 (a) Polished, unreacted surface of $\mathrm{Zn}$ pieces. (c) Post reaction using $0.05 \mathrm{M}$ $\mathrm{CuSO}_{4}$ showing fully copper coated $\mathrm{Zn}$ pieces.

4.3. XRD patterns showing comparing the effectiveness of 0.66 moles of $\mathrm{Zn}$ pieces and $\mathrm{Mg}$ ribbon in precipitating out pure copper versus copper(I) oxide. .38

4.4. XRD patterns showing comparing the effectiveness of the different forms of $\mathrm{Mg}$ (rod, powder, and ribbon) in precipitating pure copper versus copper(I) oxide.

4.5. (a) XRD patterns showing the reducing effectiveness of $\mathrm{Mg}$ ribbon as a function of reducing moles. (b) Though $\mathrm{Zn}$ was discontinued, XRD patterns showing the reducing effectiveness of $\mathrm{Zn}$ pieces as a function of moles for further confirmation that moles of reducer impacts copper(I) oxide formation.

4.6. Purity of copper precipitated as a function of moles of $\mathrm{Mg}$ ribbon reducer under $0.05 \mathrm{M} \mathrm{CuSO}_{4}$ and 2 vol.\% acetic acid conditions.

4.7. (a) GNP-Cu powder without ultrasonication in water prior to cementation process shows heavily agglomerated nanoplatelets. (b) GNP-Cu powder that was ultrasonicated in water prior to cementation process shows further dispersion and fewer nanoplatelets.

4.8. Proposed GNP surface activation process by acetic acid. .46

4.9. (a) GNP surface activated for 1 minute shows free $\mathrm{Cu}$ precipitates, with no particles on the GNP surface. (b,c,) Increasing the surface activation to 3 minutes resulted in $\mathrm{Cu}$ particles deposited on the GNP surface.

4.10. XRD patterns of GNP-Cu powder synthesized from $0.05 \mathrm{M} \mathrm{CuSO}_{4}$ solutions varying the amount of acetic acid used from 2 vol. $\%$ to 1 vol. $\%$, resulting in more effective $\mathrm{Cu}$ precipitation when compared to $\mathrm{Cu}_{2} \mathrm{O}$.

4.11. Possible mechanism showing $\mathrm{H}_{2}$ gas evolution from $\mathrm{Mg}$ ribbon surface due to acetic acid, inhibiting cementation process and resulting in $\mathrm{Cu}_{2} \mathrm{O}$ formation .50

4.12. (a,b) SEM images showing Cu nanoparticles on the GNP surface. (c,d) TEM images showing $\mathrm{Cu}$ nanoparticles on the GNP surface. (e) SAD pattern of $\mathrm{Cu}$ 
nanoparticles (f) XRD pattern of this trial shows the main peaks of $\mathrm{Cu}$, indicating $\mathrm{Cu}$ nanoparticles were precipitated. (g) Whole area EDS indicates the presence of $\mathrm{Cu}$, and minimum impurities.

4.13. (a-d) SEM images reveal that the frequency of precipitated copper clusters around GNP diminishes as $\mathrm{CuSO}_{4}$ concentration decreases from $0.10 \mathrm{M}$ to $0.005 \mathrm{M}$.

4.14. Overall flowchart of experimental parameters. Blue color highlights the optimal conditions.

4.15. XRD patterns of pre (powder) and post (compact) SPS of GNP-Cu samples........61

4.16. SEM images of powder and fracture surfaces of consolidated (a-b) pure GNP, (c-e) GNP-Cu-1 and (f-h) GNP-Cu-2.

4.17. SEM images of the fracture surface of consolidated GNP-Cu-2 in SE (a) and BSE (b) modes, as well as EDS elemental mapping (c-d). 


\section{CHAPTER I}

\section{Introduction}

The main goal of this study was to deposit nanometer scale metal particles on the surface of 2D nanoplatelets using a controlled cementation process. As a proof of concept, Copper $(\mathrm{Cu})$ was chosen as the deposited metal (based on the activity series of metals) and Graphene Nanoplatelets (GNP) were chosen as representative 2D nanoplatelets. Specific goals of this work include precipitating nanometer scale $\mathrm{Cu}$ particles on the surface of GNP at a low concentration (approximately 5 vol.\%) while maintaining impurities at a minimal amount. Parameters investigated to reach the specific goals are the metallic reducer, acetic acid, and copper (II) sulfate concentration. The GNP with copper particles (GNP-Cu) synthesized in this study will serve as precursor material to make a nacre-like structure through spark plasma sintering (SPS) for future studies. The resulting layered structure with copper metal amidst the GNP mimics nacre, a naturally occurring composite material with excellent mechanical properties such as high toughness and tensile strength.

\subsection{Significance of this Research}

\subsubsection{Utilizing the High Surface Area of 2D Materials as an Asset}

Interest in two dimensional (2D) materials has blossomed in the recent years as shown by the rise in the research of graphene. $2 \mathrm{D}$ materials have one dimension in the nanometer range, which gives them a layered structure and restricts them in that dimension $[1,2]$. The key attractiveness of $2 \mathrm{D}$ materials lies in this dimensional restriction which as a consequence, may cause several properties to differ from the $3 \mathrm{D}$ structure $[1,3]$. Properties that may deviate from the 3D structure are as follows: mechanical, thermal, 
electronic, and optical $[1,3]$. These deviations are attributed to electron confinement as well as geometric effects that arise from the characteristic high aspect ratio of 2D materials (extreme thinness compared to larger horizontal dimensions) [1,2].

Although there is high enthusiasm for 2D materials, in particular graphene, synthesizing bulk scale materials from graphene has been limited. This is due to challenges in scalable manufacturing while retaining nano-scale properties. One of the principal challenges when using graphene is agglomeration owed to the high surface energy that exists in this material due to its high surface area, an inherent property of a 2D material geometry [4]. The central focus of this work utilizes the high surface area present in 2D materials as an asset instead of a hindrance. The large surface area of GNP will be beneficial in the metallic deposition process, allowing for greater deposition opportunities and uniformity.

\subsubsection{Graphene Nanoplatelets (GNP)}

Graphene, an allotrope of carbon, is a two-dimensional sheet of $\mathrm{sp}^{2}$ bonded carbon atoms $[1,3,4,5]$. Due to the difficulty and cost attributed to isolating the single layered graphene, multi-layered graphene such GNP are commonly used [4, 6]. GNPs consist of 10-30 sheets of graphene with a thickness of 6-8 $\mathrm{nm}$, with weak Van der Waals forces in between the 2D layers $[4,5,7]$. Though multilayered, GNP retains many of the desirable mechanical, thermal and electrical properties found in single-few layer graphene [3]. 


\subsubsection{Cementation Reaction Process}

Cementation reactions are extensively used in industry to precipitate valuable metals from waste solutions [8]. This method is advantageous due to its simple elegance and low cost. The underlying principle of the cementation reaction process is based on the activity series of metals. This process employs a more reactive metal to displace a less reactive metal ion from its salt solution [9]. Equation 1 illustrates the detailed chemical reaction considered. The more reactive metal, magnesium $(\mathrm{Mg})$ displaces the copper $(\mathrm{Cu})$ metal from the copper(II) sulfate $\left(\mathrm{CuSO}_{4}\right)$ solution:

$$
\mathrm{Cu}^{2+} \mathrm{SO}_{4}{ }^{2-}{ }_{(\mathrm{qq})}+\mathrm{Mg}^{0}(\mathrm{~s}) \Rightarrow \mathrm{Mg}^{2+} \mathrm{SO}_{4}{ }^{2-}{ }_{(\mathrm{aq})}+\mathrm{Cu}^{0}{ }_{(\mathrm{s})}
$$

This is a reduction-oxidation reaction in which the more reactive metal is the reducer and is oxidized (loses electrons) while the displaced metal is reduced (gains electrons). The copper ion is reduced to its ground state due to the difference in electrode potentials between the reducer and the metal in the salt solution. [8].

Cementation reactions have been studied as a viable option to coat carbon fibers and graphite particles for composite applications [9-11]. As exemplified by equations 1, the cementation process produces pure metal precipitates. In this work, glacial acetic acid is used as a surface activator in order for the pure metal particles to be deposited onto the GNP surface instead of freely precipitating out into solution [9-11]. Figure 1.1 illustrates the cementation reaction process that occurs in this study by showing the reduction of the copper ion by the metal reducer $(\mathrm{Mg})$ on the surface of the GNP itself. 

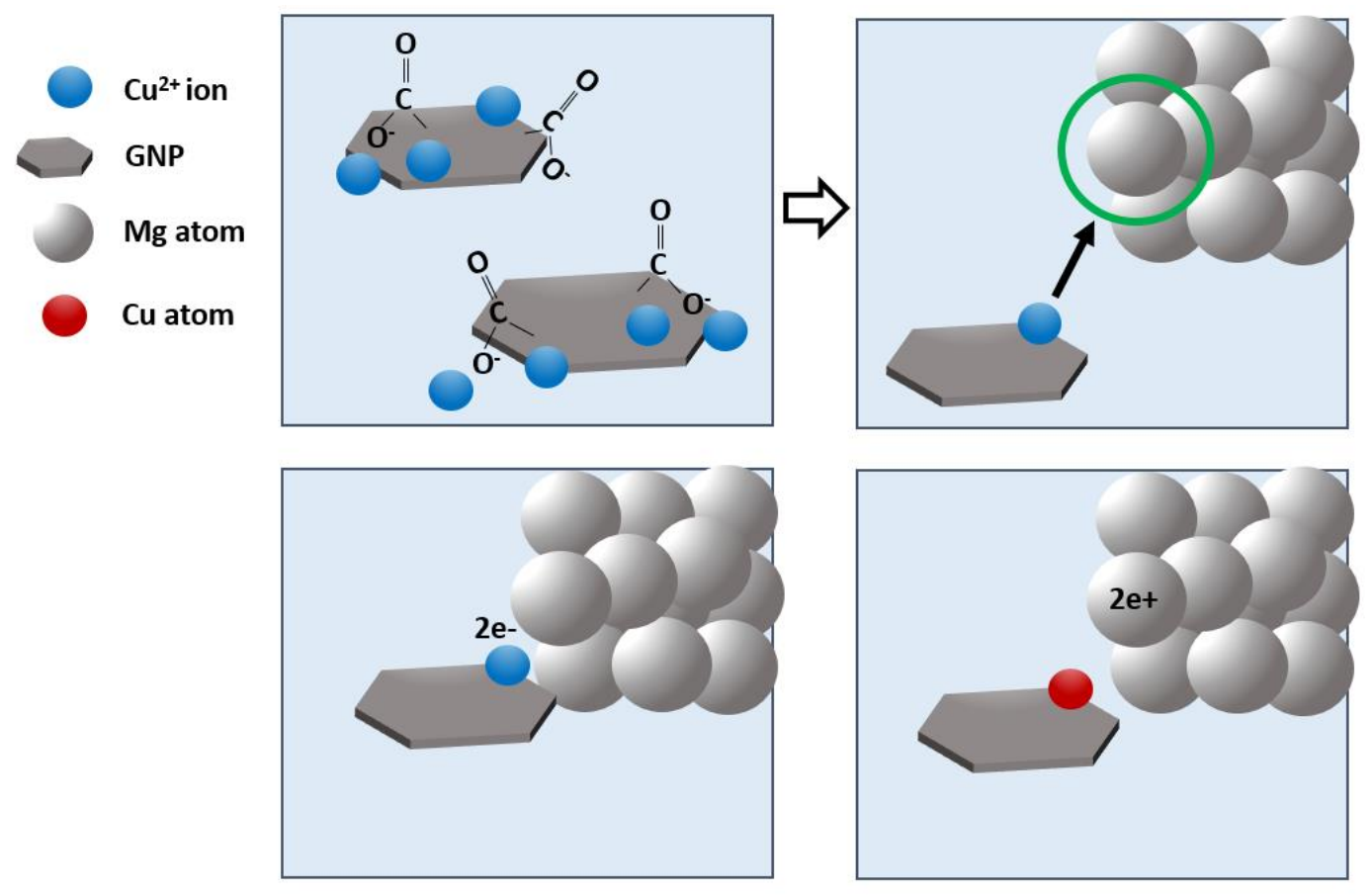

Figure 1.1. Schematic of Cementation Reaction. a) Glacial acetic acid used to activate the GNP surface, represented as black ovals. b-d) Reduction of copper ions on the GNP surface by reducer metal.

\subsubsection{Natural Nacre}

The GNPs with copper particle deposits (GNP-Cu) synthesized in this work are precursor materials in order to then prepare a "nacre"-like structure. Nacre is a hybrid biocomposite of 95 vol.\% ceramic $\left(\mathrm{CaCO}_{3}\right)$ and 5 vol.\% organic matrices found at the interfaces $[12,13]$.

Nacre is a naturally occurring composite material with excellent widely-recognized mechanical properties such as high fracture toughness (across: 3.3-4.6 $\mathrm{MPa} \mathrm{m}^{-1 / 2}$ and along 3.7-5.0 $\mathrm{MPa} \mathrm{m}^{-1 / 2}$ ) and mechanical strength under tensile loading (across: 90-167 MPa) $[13,14]$. One would expect a material with high volume percentage of a ceramic component to be brittle, however studies on nacre have determined it to be about 3000 times tougher than monolithic $\mathrm{CaCO}_{3}[12,13]$. 
In this study, the copper particles to be deposited are analogous to the organic matrices of nacre, while the GNP will serve as the major component of the composite. The GNP-Cu powder synthesized in this work will then be sintered via spark plasma sintering (SPS)to mimic a nacre-like structure. SPS is a sintering technique that uses high pressure and temperature, while pulsing current through the material during the consolidation process [15]. This results in densification and enhanced bonding between the dissimilar phases [15]. The resulting structure of nanometer scale copper metallic layers between GNPs mimics the natural nacre structure.

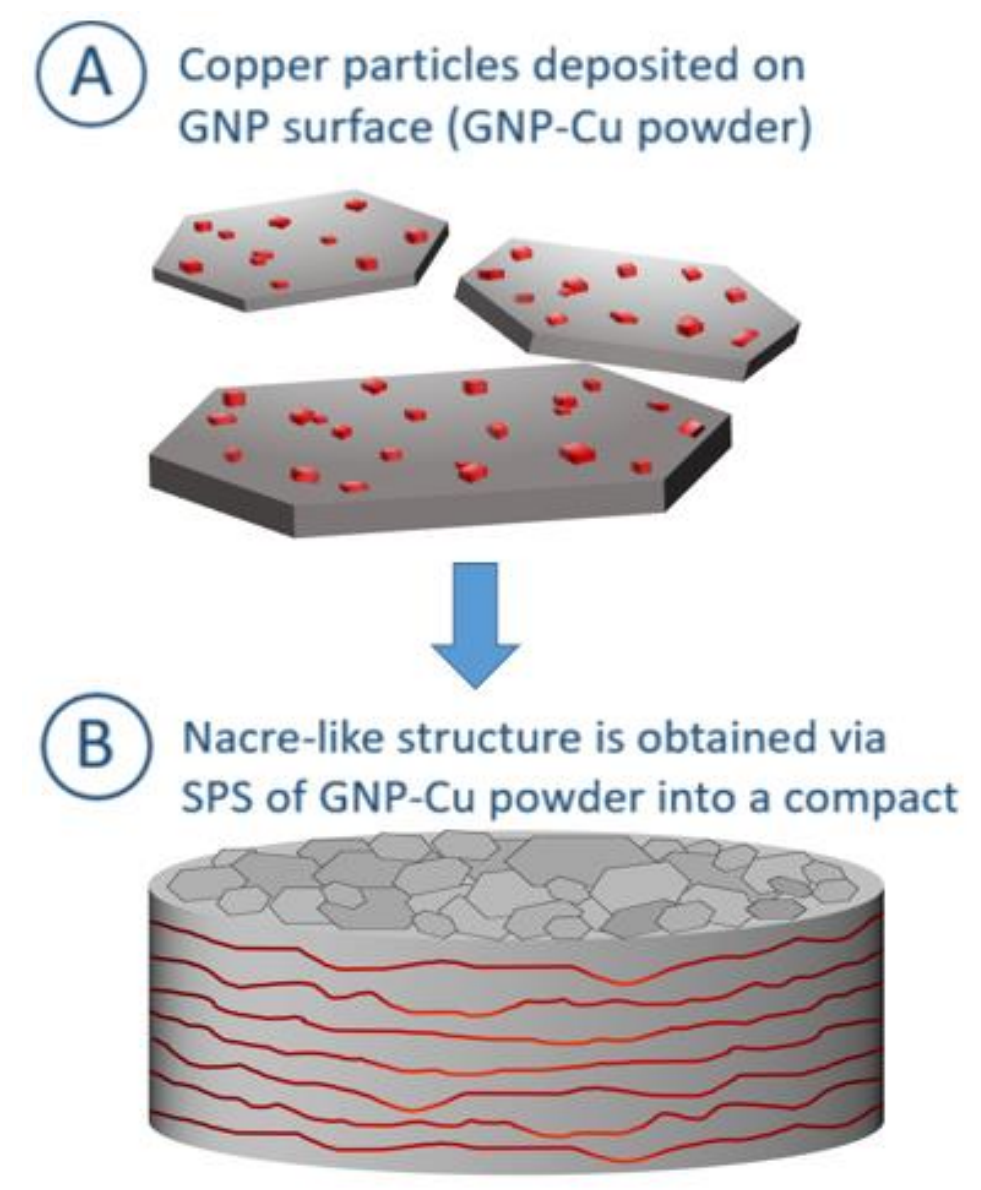

Figure 1.2. (a) Schematic of GNP (represented by grey ovals) with copper particles (represented by red cubes) deposited on its surface post cementation reaction. (b) Schematic of GNP-Cu compact post SPS shows a layered nacre-like structure of nanometer thin copper metal amidst GNPs. 


\subsection{Research Objectives}

The overall objective of this work is to optimally synthesize a uniform deposition of nanometer scale copper particles onto the surface of Graphene NanoPlatelets (GNP), a two dimensional (2D) material, via a cementation reaction process. Also desired is a low copper concentration (5 vol.\%) and minimum impurities.

The following reveals the approaches taken to meet the objective presented:

- Effective deposition of copper particles throughout the GNP surface requires reduction of GNP agglomeration via sonication.

- Parametric studies were carried out to optimize synthesis conditions in order to achieve the overall objective of this work. Parameters varied are as follows:

O Metal Reducer: type, form and quantity - affects the purity of copper precipitates by controlling the formation of copper oxide and $\mathrm{Mg}$ impurities.

- Acetic Acid

- Surface activation time - affects particle deposition onto the GNP surface.

- Vol. \% - affects the purity of copper precipitates by controlling the formation of copper oxide.

- Copper(II) sulfate concentration - affects quantity and size of pure copper particles deposited onto the GNP surface.

The subsequent chapter will present a review of Graphene, cementation reactions and similar processes used for metallic particle deposition, as well as literature studies seeking to mimic the nacre-like structure. Chapter 3 discusses the materials and experimental 
methods used to synthesize and characterize the GNP with copper deposits (GNP-Cu) composite material. Chapter 4 examines synthesis and characterization results with the discussion of the underlying science. Chapter 5 presents the major conclusion of this work and Chapter 6 suggests recommendations for further research in this area. 


\section{CHAPTER II}

\section{Literature Review}

This chapter will present a literature review of: (i) Graphene based 2D material, and (ii) Processes used for metallic nanoparticle deposition on 2D materials. A brief review of other studies seeking to mimic the nacre-like structures via various techniques is also presented

\subsection{Graphene}

Graphene is an integral component of this work as it is the major constituent of the composite material to be produced and studied. Graphene, a single layer of graphite, is a two-dimensional sheet of $\mathrm{sp}^{2}$ bonded carbon atoms densely packed in a hexagonal crystal lattice $[1,3,16,17]$. Figure 2.1 shows high resolution images of graphene from transmission electron microscopy (TEM) and scanning tunneling microscopy (STM) [19, 20]. These images clearly illustrate the hexagonal, honeycomb-like structure of graphene. Graphene's hexagonal structure is of importance since it serves as a building block for other carbon based allotropes [3]. It forms graphite (3D) when stacked, nanotubes (1D) when rolled, and fullerenes (0D) when wrapped [3]. 

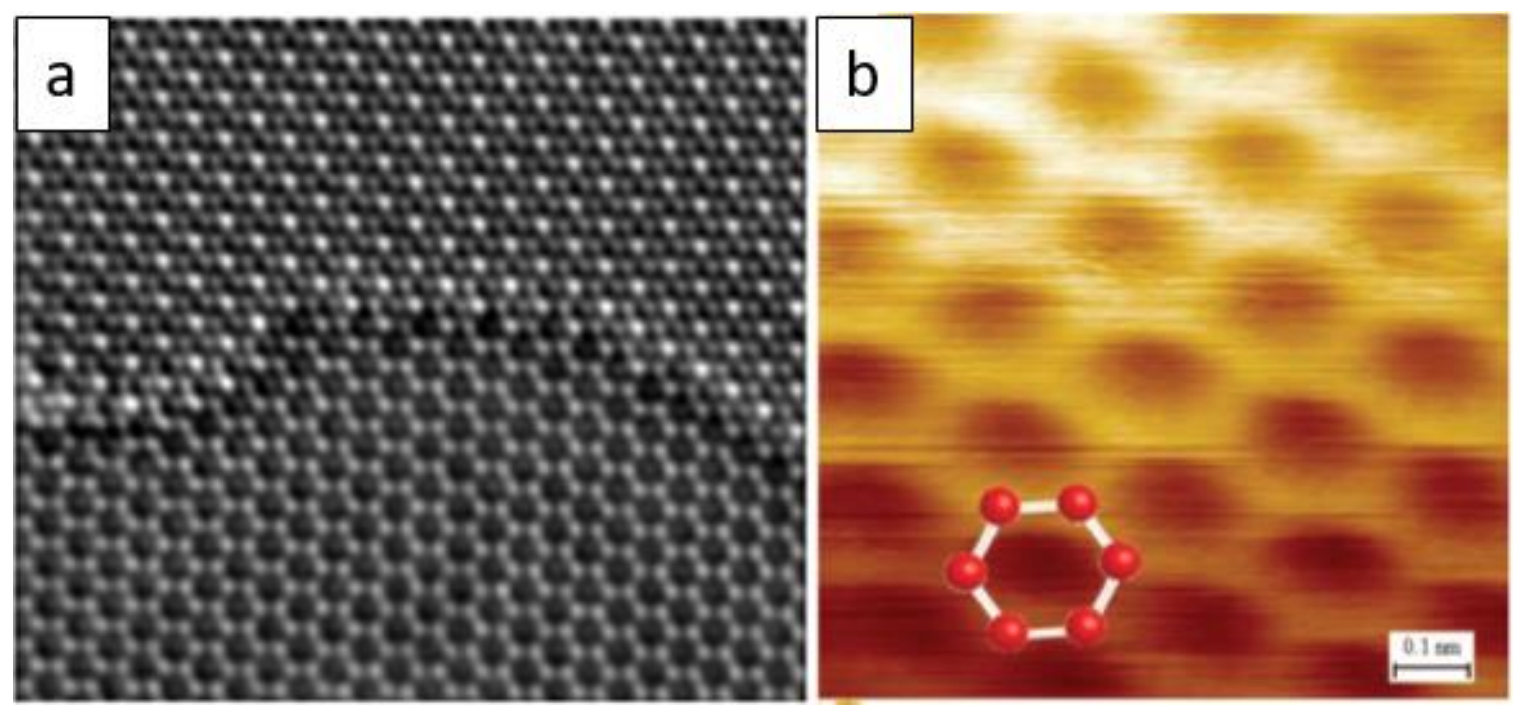

Figure 2.1 (a) HRTEM image of single and bilayer graphene [18]. (b) HRSTM image of mechanically exfoliated single-layer graphite showing honeycomb structure [19].

Studies of graphite were being employed using less and less layers of graphite [3]. Until single layered graphene could be consistently attained and characterized, properties of one-atom thick layer of $\mathrm{sp}^{2}$ carbon were predicted but unable to be investigated [1]. In the past few decades, extensive effort was put forth in attaining single layers of graphite, also known as graphene sheet [1]. Synthesis of monolayer graphite was attempted as early as 1975 by Lang et al. via thermal decomposition of carbon [20]. However, due to lack of consistency in obtaining monolayer graphite, this method was not widely pursued. In 2004, an exponentially growing scientific interest was sparked after Novoselov and Geim at Manchester University, UK were able to directly observe isolated graphene layers via an exfoliation technique [21].

The main methods for procuring graphene are mechanical exfoliation, epitaxial growth, graphene oxide reduction and molecular approaches [22]. The Manchester group was able to consistently isolate graphene from graphite using micromechanical exfoliation 
method $[3,17,21]$. This method removed successive layers of highly oriented pyrolytic graphite (HOPG) by using adhesive tape, which was then pressed upon a substrate to deposit the attached graphite flakes [3, 23]. Though the obtained flakes are multilayered graphite, due to the Van der Waals attraction between graphite layers and substrate, a single layer of graphite is delaminated simply by lifting the tape $[3,21]$. By using a top-down approach to obtain graphene, the Manchester group was able to bypass possible issues of stability associated with small crystallites [17].

Graphite is simply stacked layers of graphene bonded together by weak Van der Waals forces, which possess an interaction energy of approximately $2 \mathrm{ev} / \mathrm{nm}^{2}$. This makes the force needed to exfoliate graphite in the order of $300 \mathrm{nN} / \mu \mathrm{m}^{2}$ [24]. The weak force required for graphite exfoliation was easily overcome using adhesive tape as was carried out by aforementioned the Manchester group [24]. Possible contaminants due to adhesive glue may be overcome via heat treatment [24]. This method was further modified by other groups by using differing substrates in order to control the bonding between the graphite and substrate $[25,26]$. Exfoliation may also be carried out in solution, as shown by Blake et al. by using of $N$-methyl-pyrrolidone, and through the use of surfactants such as sodium dodecylbenzene sulfonate (SDBS) and sodium cholate [27-30].

Another common method to obtain single or few layered graphene is through thermal chemical vapor deposition (CVD) techniques. The first experiment to successfully synthesize few layer graphene through this method took place in 2006 [31]. Since then, this process has been widely investigated. Most often, the growth of graphene via thermal CVD occurs on a nickel substrate or film, a metal with high carbon solubility [31-33]. This occurs via the diffusion of carbon into the metal surface at a certain growth temperature, 
followed by subsequent cooling which causes the precipitation of carbon from the metal surface $[34,35]$. The thickness and crystal ordering of the carbon layer precipitated is controlled by the cooling rate used, the concentration of carbon diffused into metal, as well as the thickness of the metal layer [23]. Graphene may also be grown on metals with low carbon solubility such as copper. With these substrates, the process becomes surfacecatalyzed and self-limiting, rather than a precipitation process as is the case with nickel $[16,23,36]$. Variations of this method includes plasma enhanced CVD, which allows graphene to be quickly grown $(<5 \mathrm{~min})$ on a variety of substrates including transition metals and oxides [23]. In this method, graphene growth occurs vertically due to the direction of the plasma electric field [37-38].

Graphene may also be obtained from chemically reducing graphene oxide (GO) by using reducing agents such as hydrazine and sodium borohydrate [23]. As well as through molecular approaches by altering carbon allotropes such as carbon nanotubes (CNT). One of the ways graphene can be obtained from CNTs is via plasma etching as shown by Jiao et al. in 2009 [40]. This causes the cylindrical CNT to "unzip" into graphene layers.

Due to the difficulty and cost attributed to the methods used to isolate single layered graphene as illustrated above, multi-layered graphene such GNP are commonly used [4, 6]. GNPs have a thickness of 6-8 $\mathrm{nm}$ and are comprised of 10-30 sheets of graphene. Weak Van der Waals forces are observed between the 2D layers [4,5,7]. Though GNP are multilayered, it still retains many of the desirable mechanical, thermal and electrical properties found in single to few layered graphene [3]. 


\subsection{Nanoparticle (NP) Metallic Deposition on 2D Materials}

Deposition or attachment of particles onto 2D materials, most often graphene and graphene oxide, is a practiced approach to reduce aggregation between sheets produced by strong Van der Waals interactions [41]. Particles deposited are predominantly in the nanometer scale and of inorganic nature, such as metals, metal oxides, metal sulfides. Though polymer coated graphene platelets have also been investigated for the same purpose [41]. Using inorganic particles as "spacers" is not only advantageous for reducing agglomeration, but has also lead to performance enhancement in sensor applications [42]. Various chemical and physical techniques have been used in order to deposit nanoparticles onto the surface of 2D materials. These are summarized in Table 2.1. Most commonly used techniques are in situ chemical reduction methods using various reducers, and coprecipitation. Physical techniques used include electrodeposition and laser irradiation.

Table 2.1. Techniques and Outcomes for Metallic Nanoparticle (NP) Deposition

\begin{tabular}{|c|c|c|c|c|}
\hline $\begin{array}{l}\text { Deposition } \\
\text { Techniques }\end{array}$ & $\begin{array}{l}\text { 2D materials } \\
\text { used and NPs } \\
\text { deposited: }\end{array}$ & $\begin{array}{c}\text { Other Materials } \\
\text { Used }\end{array}$ & Outcomes & Ref \\
\hline $\begin{array}{l}\text { Chemical } \\
\text { Reduction }\end{array}$ & $\begin{array}{c}\text { 2D material: } \\
\text { - } \mathrm{GO} \\
\text { - } \mathrm{rGO} \\
\text { - } \mathrm{GNS} \\
\text { NPs deposited: } \\
\text { - } \mathrm{Au} \\
\text { - } \mathrm{Ag} \\
\text { - } \mathrm{Pt} \\
\text { - } \mathrm{Pd} \\
\text { - } \mathrm{Cu}\end{array}$ & $\begin{array}{l}\text { Reducers: } \\
\text { - ethylene } \\
\text { glycol } \\
\text { - propanol-2 } \\
\text { - } \mathrm{NaBH}_{4} \\
\text { - } \mathrm{NaOH} \\
\text { - chitosan } \\
\text { - hydrazine }\end{array}$ & $\begin{array}{l}\text { All particles } \\
\text { precipitated in the } \\
\text { nanometer range. } \\
\text { Particle size: } \\
\text { Glycol: } \\
\text { Pt: Avg. } 2.75 \mathrm{~nm} \\
\text { Au: Range } 10-20 \mathrm{~nm} \\
\text { Ag: Range } 3-6 \mathrm{~nm} \\
\text { NaBH} 4: \\
\text { Ag: Avg. } 13 \mathrm{~nm} \\
\mathrm{Cu}: \text { Range } 5-25 \mathrm{~nm} \\
\mathrm{Pd}: \text { Avg. } 30-40 \mathrm{~nm}\end{array}$ & $44-51$ \\
\hline
\end{tabular}




\begin{tabular}{|c|c|c|c|c|}
\hline $\begin{array}{l}\text { Co- } \\
\text { precipitation }\end{array}$ & $\begin{array}{c}\text { 2D material: } \\
\text { - } \mathrm{GO} \\
\text { - } \mathrm{BNNS} \\
\text { NPs deposited: } \\
\text { - } \mathrm{Fe}_{3} \mathrm{O}_{4}\end{array}$ & $\begin{array}{l}\text { Ferrous chloride } \\
\text { and ferric } \\
\text { chloride }\end{array}$ & $\begin{array}{l}\text { This method led to } \\
\text { more complete and } \\
\text { uniform coatings } \\
\text { instead of separate } \\
\text { NPs. }\end{array}$ & $\begin{array}{l}52 \\
53\end{array}$ \\
\hline $\begin{array}{l}\text { Electro- } \\
\text { deposition }\end{array}$ & $\begin{array}{cc}\text { 2D material: } \\
\text { - } \quad \text { rGO } \\
\text { - } \quad \text { functio } \\
\text { nalized } \\
\text { rGO } \\
\text { NPs deposited: } \\
\text { - } \mathrm{Au}\end{array}$ & & $\begin{array}{l}\text { Au particles and } \\
\text { dentrites deposited } \\
\text { throughout rGO and } \\
\text { functionalized rGO } \\
\text { surfaces. }\end{array}$ & $54-56$ \\
\hline $\begin{array}{l}\text { Electroless } \\
\text { Deposition }\end{array}$ & $\begin{array}{cl}\text { 2D material: } \\
\text { - } \quad \text { GS } \\
\text { (graphe } \\
\text { ne } \\
\text { sheets) } \\
\text { NPs deposited: } \\
\text { - } \mathrm{Au} \\
\text { - } \mathrm{Ag} \\
\text { - } \mathrm{Pd} \\
\text { - } \mathrm{Pt}\end{array}$ & & $\begin{array}{l}\text { Homogeneous and } \\
\text { uniform NP deposition } \\
\text { throughout GS. } \\
\text { Ex. Pt NP diameter } \\
\text { range is } 8-40 \mathrm{~nm} \\
\text { (particle size is } \\
\text { dependent on reaction } \\
\text { time, which was varied } \\
\text { from } 5 \mathrm{~s} \text { to } 20 \mathrm{~s} \text { ) }\end{array}$ & 57 \\
\hline $\begin{array}{l}\text { Laser } \\
\text { irradiation }\end{array}$ & $\begin{array}{c}\text { 2D material: } \\
\text { - GO } \\
\text { film } \\
\text { NPs deposited: } \\
\text { - Ag }\end{array}$ & & $\begin{array}{l}\text { Varying intensity of } \\
\text { laser beam resulted in } \\
\text { controlling of } \mathrm{Ag} \\
\text { particle shape, size and } \\
\text { distribution. }\end{array}$ & 58 \\
\hline
\end{tabular}

\subsubsection{Chemical Reduction Techniques}

Similar to cementation reactions are in situ chemical reduction reactions, also called neutralization reactions. Unlike cementation reactions which use a sacrificial metal, this method uses hydroxides, alcohols, and/or acid solutions to neutralize the metal ions in solutions and precipitate out either in solution or onto the surface of 2D materials [43-53]. Chemical reduction methods are often used to not only reduce the metal ions, but simultaneously reduce graphene oxide (GO) as well. Disadvantages of this approach 
include resulting high moisture content and contaminants such as metal hydroxides and metal salts included along with the precipitated pure metal [43]. Furthermore, controlling reaction completion is difficult. Many studies have to wash, centrifuge or sonicate the excess precipitated particles away, resulting in planning accurate volume percentage of materials difficult to obtain.

Ethylene glycol is one of the most common reducers used in NP metallic deposition due to its mildly reducing and environmentally friendly properties. Studies have used graphene oxide (GO) or graphene nanosheets (GNS) as a precursor in glycol reducing conditions as well as propanol-2 conditions, which is less used. Metallic NPs precipitated and deposited onto GO or GNS using this method include Au, Ag, Pt, Pd [44-48].

Other reducers that are commonly used in chemical reduction synthesis, are sodium borohydride $\left(\mathrm{NaBH}_{4}\right)$ and sodium hydroxide $(\mathrm{NaOH})$, though toxic and highly corrosive respectively. Investigations have used these reducers either separately or in conjunction with ethylene glycol in order to obtain $\mathrm{Au}, \mathrm{Ag}, \mathrm{Cu}$ and Pt NPs on top of rGO and GNS surfaces $[46,49,50]$.

Of note is Guo et al.'s investigation in which chitosan, a polysaccharide, was employed not only as a reducer but also stabilizer (control NP growth rate and nucleation) to prepare $\mathrm{Au}$ and Ag NPs on rGO. This shows that an organic material such as a polysaccharide, which is biodegradable and biocompatible, can serve as a viable approach in metal ions and rGO reducing processes. Hydrazine has shown efficient reductive effect though it is highly toxic $[50,51]$.

Co-precipitation is yet another tactic to synthesize metallic NPs coatings and depositions. In particular, iron oxide $\left(\mathrm{Fe}_{3} \mathrm{O}_{4}\right)$ NPs coatings on the surfaces of $\mathrm{GO}$ and Boron 
Nitride Nanoplatelets (BNNP) sheets were considered [52, 53]. In both studies, the surfaces of the 2D materials had to be activated by the help of coupling agents, APTES (3aminopropyltrimethoxysilane) and PSS (Poly(sodium 4-styrenesulfonate), respectively.
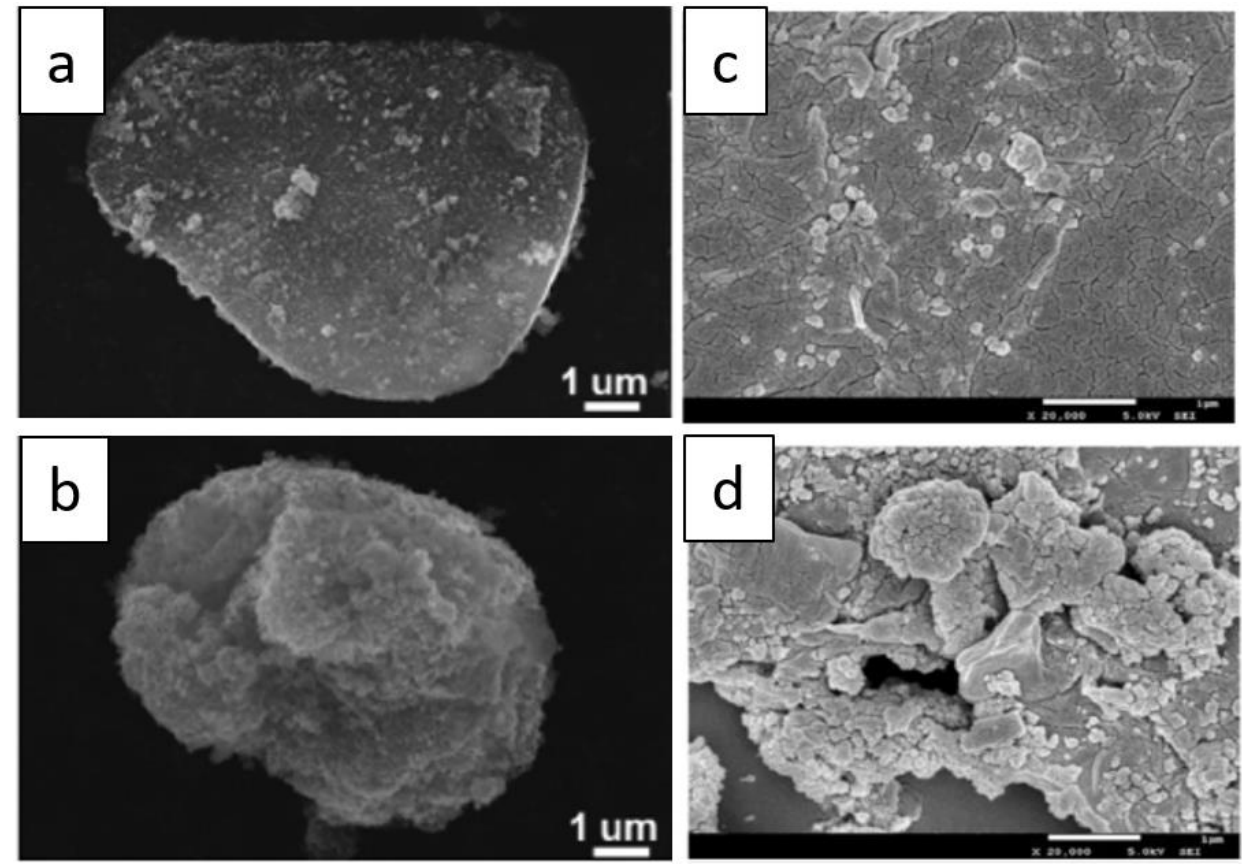

Figure 2.2. (a-c) SEM images showing varied morphologies and quantity of $\mathrm{Fe}_{3} \mathrm{O}_{4} \mathrm{NPs}$ deposited onto (a,b) BNNP and (c) GO via co-precipitation. Images (a) and (b) shows the variance in coating when the weight $\%$ of iron oxide NP is changed from (a) $30 \%$ to (b) $100 \%$. Images (c) and (d) shows iron oxide:GO ratio of 1:6 and 1:3 respectively $[52,53]$.

\subsubsection{Electrochemical Techniques}

Electrodeposition technique has been used to deposit metallic NP onto the surface of reduced graphene oxide (rGO) for various chemical and biological sensor applications, most often for drugs, glucose and macromolecules such as peptides [54-56]. Though most approaches for graphene/NP nanocomposites are through wet chemistry techniques previously detailed, these have multiple steps and may be time-consuming. Advantages to using electrodeposition as an alternative technique lies in its shorter deposition length, and 
high purity and uniformity of products. Au NP has been deposited on the surface of rGO in multiple investigations for sensor applications [54-56]. Few studies have used functionalized rGO surfaces. Gold NPs electrodeposited of varied morphologies onto rGO functionalized surfaces with $\mathrm{NH}_{2}$ and globular protein $\beta$-lactoglobulin (BLG) respectively can be observed in Figure 2.3.

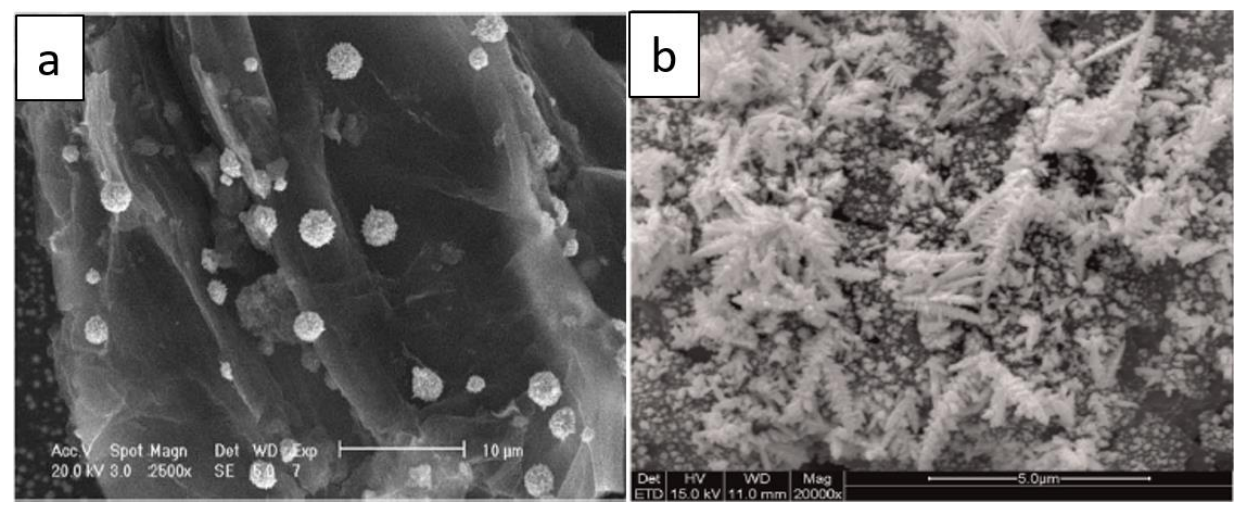

Figure 2.3. (a,b) SEM images showing varied morphologies of AuNPs electrodeposited onto the (a) $\mathrm{NH}_{2}$ and (b) BLG functionalized $\mathrm{rGO}$ surface via electrodeposition. $[55,56]$

Interestingly, Liu et al. have taken advantage of the conductivity of graphene sheets (GS) and the difference in redox potential of metal substrate and metal ions in order to synthesize metal-graphene composites by electroless deposition. This was done by placing GS into a metal substrate suspension first. This metal foil supported GS substrate was then submerged into a metal ion solution where the metal ions were reduced into metallic $(\mathrm{Ag}$, $\mathrm{Au}, \mathrm{Pd}, \mathrm{Pt}$ ) nanoparticles on the GS surface [57].

\subsubsection{Laser Irradiation}

Laser irradiation is a simplistic approach for achieving local reduction metallic ions into metal NPs for deposition onto 2D surfaces. Teoh et al. have taken advantage of this 
method by using a focused laser beam to reduce Ag ions into Ag NPs on a GO film that was submerged in $\mathrm{AgNO}_{3}$ solution, resulting in $\mathrm{Ag}$ NPs attached to the GO surface [58].

Studies discussed above have led to the formation of composites with 2D materials and metal nanoparticle deposition via multiple approaches. However, to the best of this author's knowledge, metal nanoparticles deposition onto $2 D$ materials via cementation reactions has not yet been explored, which is the main objective of this study.

\subsubsection{Cementation Reactions}

Cementation reactions have been studied as a viable option to coat carbon fibers and graphite particles for composite applications [9-11]. Uniform coatings of copper, nickel, and cobalt have been previously investigated in addition to using magnesium, aluminum, zinc, and iron as reducers [9]. Previous investigations concluded that the thickness, uniformity and adherence of the metal coating was dependent upon the concentrations of metal ion solution, surface activator, reducer type and size. Furthermore, the kinetics of cementation reactions have also been investigated by varying metal salt solution concentration, reducer metal concentration and $\mathrm{pH}[8,59-61]$.

A major processing parameter of cementation reactions include the type of metal ion to be reduced into its metallic state. Furthermore, by using surface activators, one may use this process to then obtain various metal coatings on numerous materials. Thus by varying the constituents used and their concentration, varying composites may be produced. In particular, through rapid consolidation of coated particles, using processes such as spark plasma sintering (SPS), resulting lamellar structures with metal amidst the 
particles may be fabricated. By varying constituent materials, composites may be made to mimic nacre, a naturally occurring composite material with a layered structure.

\subsection{Artificial Nacre}

The aim of this work is to synthesize precursor material made of GNPs with copper particle deposits (GNP-Cu) in order to prepare a "nacre"-like structure in the future. Nacre, a hybrid biocomposite, is 95 vol. \% calcium carbonate $\left(\mathrm{CaCO}_{3}\right)$ in the form of polygonal tablets, and 5 vol. \% of organic matrices composed of proteins and polysaccharides 10-50 $\mathrm{nm}$ in size found at the interfaces $[12,13]$.

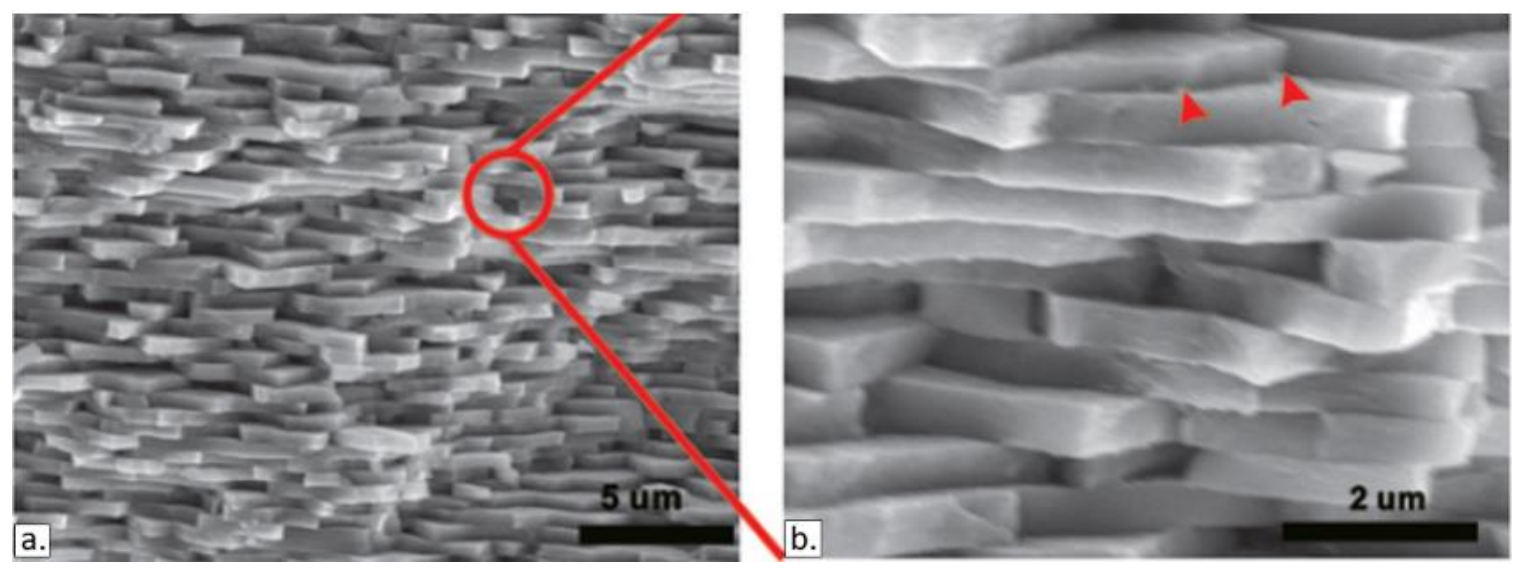

Figure 2.4. (a) SEM of a cross section of natural nacre. (b) close up of same image with arrows showing the interface between $\mathrm{CaCO}_{3}$ and organic matrices. [14]

Due to nacre's admirable mechanical properties such as high fracture toughness and mechanical strength under tensile loading, nacre inspired research seeking to mimic the structural and mechanical properties of this natural composite has recently grown [1214]. Challenges of this endeavor include duplicating this material's well defined structure as well as bulk fabrication to be used in various applications such as in the biomedical and electronics fields $[13,14]$. Various techniques have been used to produce artificial nacre, 
usually made with a ceramic matrix and polymer reinforcement, are outlined in Table 2.2 below.

Table 2.2. Techniques employed to synthesize nacre-like structures

\begin{tabular}{|c|c|c|}
\hline Techniques & Material System & $\operatorname{Ref}$. \\
\hline Electrophoretic Deposition & Montmorillonite (clay) and acrylamide & $\begin{array}{l}62, \\
63\end{array}$ \\
\hline \multirow[t]{2}{*}{ Sintering } & $\begin{array}{l}\text { Partially stabilized zirconia (PSZ) sheets with } \\
\text { adhesive (Dymax 1180-M) in between }\end{array}$ & \multirow[t]{2}{*}{$\begin{array}{l}64 \\
65\end{array}$} \\
\hline & Ag coated glass powder & \\
\hline \multirow[t]{2}{*}{ Self-assembly } & $\begin{array}{l}\text { Indium-tin-oxide films and acrylate-based } \\
\text { polymer }\end{array}$ & \multirow[t]{2}{*}{$\begin{array}{l}66 \\
67\end{array}$} \\
\hline & $\begin{array}{l}\text { Boron nitride nanosheets and poly(vinyl } \\
\text { alcohol) }\end{array}$ & \\
\hline Sputtering & $\mathrm{TiN} / \mathrm{Pt}$ & 68 \\
\hline $\begin{array}{l}\text { Physical Methods for } \\
\text { achieving lamellar } \\
\text { structure: } \\
\text { - Centrifugation } \\
\text { - Shear mixing } \\
\text { - Sedimentation } \\
\text { - Dipping } \\
\text { - Spinning cylinder } \\
\text { - Spinning plate } \\
\end{array}$ & Talc and polyvinylacetate & 69 \\
\hline Freeze casting & Alumina platelets and silica, calcia & 70,71 \\
\hline Layer by layer deposition & $\begin{array}{l}\text { Au nanoparticles and polymer } \\
\text { (poly(diallylmetylammonium)) }\end{array}$ & 72 \\
\hline
\end{tabular}

Lin et al. have tried to mimic nacre by using a two-step method of a hydrothermal process then subsequent electrophoretic deposition using layered silicate (montmorillonite platelets with high aspect ratio) and a polymer, acrylic anodic electrophoretic resin (AAER) polymer [62]. This group found via nanoindentation that despite the low organic content of approximately $8 \mathrm{wt} . \%$, the reduced Young's modulus of the composite film was successfully enhanced to $5.0 \pm 1.0 \mathrm{GPa}$ when compared to layered silicate film alone $(2.9$ $\pm 0.4 \mathrm{GPa}$ ). Similarly, Long et al. have also created a nacre-like composite via electrophoretic deposition using montmorillonite and polyacrylamide [63]. Results showed 
a highly organized "brick and mortar" structure very similar to natural nacre and the group has reported improved mechanical properties when compared to pure montmorillonite. Hardness of the composite was found to be $0.95 \mathrm{GPa}$ and Young's modulus was 16.92 $\mathrm{GPa}$, which is an increase when compared to the values obtained from pure montmorillonite, $0.20 \mathrm{GPa}$ and $2.92 \mathrm{GPa}$ respectively.

Another approach was bonding thin sintered ceramic sheets of zirconia stabilized with $5 \mathrm{~mol} \%$ yttrium oxide (PSZ) with thin adhesive layers of an UV-light cured adhesive (Dymax 1180-M) at a very low volume \% (below 6\%) [64]. This group reported improved crack resistance, significant stiffness (96 GPa) and bending strength (297 MPa). The sintering approach was also undertaken by Kakisawa et al. whom rolled and aligned glass powder with a silver coating to fabricate a dense nacre-like composite [65]. They have reported non-brittle fracture behavior in a bending test as well as resistance to crack extension due to crack deflection as well as interface delamination.

X. Zhang et al. have created laminated thin films of indium-tin-oxide (ITO) and an acrylate-based polymer, poly(tripropylene glycol diacrylate) by evaporation-induced selfassembly method on a quartz glass substrate [66]. The nacre-like structure of this nanocomposite film was observed via TEM. Likewise, vacuum-assisted self-assembly technique was used by Zeng et al. to create artificial nacre "paper" using boron nitride nanosheets as the matrix and a polymer, poly(vinyl alcohol) [67]. The papers created show excellent tensile strength (125.2 MPa) and display a 30\% higher toughness than that of natural nacre [14].

Nacre-like thin films using the polymer as the matrix instead of reinforcement was created by Abba et al. using the biopolymer chitosan and alumina platelets as the 
reinforcement via shear mixing [13]. Improved modulus of the film was reported when the volume fraction of alumina increased from $0 \%$ to $15 \%$, while tensile strength was reduced when the volume fraction of alumina was higher than $5 \%$. Furthermore, this group also studied the effect of relative humidity on the mechanical properties of the hybrid film. They have shown that increasing humidity (from $36 \%$ to $75 \%$ ) significantly decreased the modulus and strength of the thin film (by 45\%), which suggests applications in specific conditions or environments.

Physical approaches such as sputtering metal onto ceramic substrate are other successful approaches to mimicking nacre [68]. Various physical methods were investigated by Almqvist et al. The techniques used to properly align the ceramic tablets are listed in order of most to least effective as follows: such as centrifugation, shearing, sedimentation, dip coating, spinning cylinder, spinning plate and shearing plates [69]. The physics of ice formation may be taken advantage of in order to freeze cast ceramic slurries, creating ceramic scaffolds to then be filled by polymers or metals and metal oxides [70, 71]. Also if interest is Andres et al's approach in which layer-by-layer assembly of films were achieved by inkjet deposition using negatively charged gold nanoparticles and positively charged polymer (Poly(diallylmetylammonium) [72].

As discussed, artificial nacre has been created using various methods using several components, most commonly ceramic and polymer components. Though these synthesized materials show interesting mechanical properties, the improvement seen in the level of toughness and highly organized structure observed still remain inferior when compared to natural nacre [73]. Furthermore, many of the techniques described above are only conceivable in the small scale, thus making these materials for production unfeasible. 
Unlike most investigations in this area, this work aims to mimic nacre using Graphene Nanoplaletets (GNP) and copper nanoparticles in a novel manner that may also be applicable in large scale by consolidation via spark plasma sintering. 


\section{CHAPTER III}

\section{Materials and Experimental Methods}

This chapter discusses in detail the materials and experimental methods used to synthesize and characterize the GNP with copper deposits (GNP-Cu) composite powder. The materials, powder processing, cementation reaction details and processing parameters are presented. Also presented are the various characterization techniques used to investigate the microstructure and phases in the composite powder. The precursor powder was also sintered via spark plasma sintering (SPS) to evaluate the feasibility of composite powder for preparing a nacre-like structure. A preliminary characterization of sintered GNP-Cu compact is also shown.

\subsection{Materials}

\subsubsection{Graphene Nanoplatelets (GNP)}

Graphene Nanoplatelets (xGNP-M-5) were acquired from XG Sciences (Lansing, MI, USA). GNP particles have an average diameter of $15 \mu \mathrm{m}$, making their relative surface area to be $120-150 \mathrm{~m}^{2} / \mathrm{g}$ [7]. GNPs have a thickness in the range of $6-8$ nanometers. Considering that each layer of graphene is approximately $0.35-0.37 \mathrm{~nm}$ thick, a typical GNP particle is composited of approximately 20 sheets of graphene [16]. On the edges of the GNPs, few functional groups may be found such as $0.075 \%$ ether, $0.035 \%$ carboxyl, $0.03 \%$ hydroxyl groups [4]. GNP starting powder morphology is observed in Figure 3.1, which shows the folds and wrinkles present in the top surface (Fig 3.1a) and a cross section image of few GNPs stacked (Fig 3.1b) [4]. 


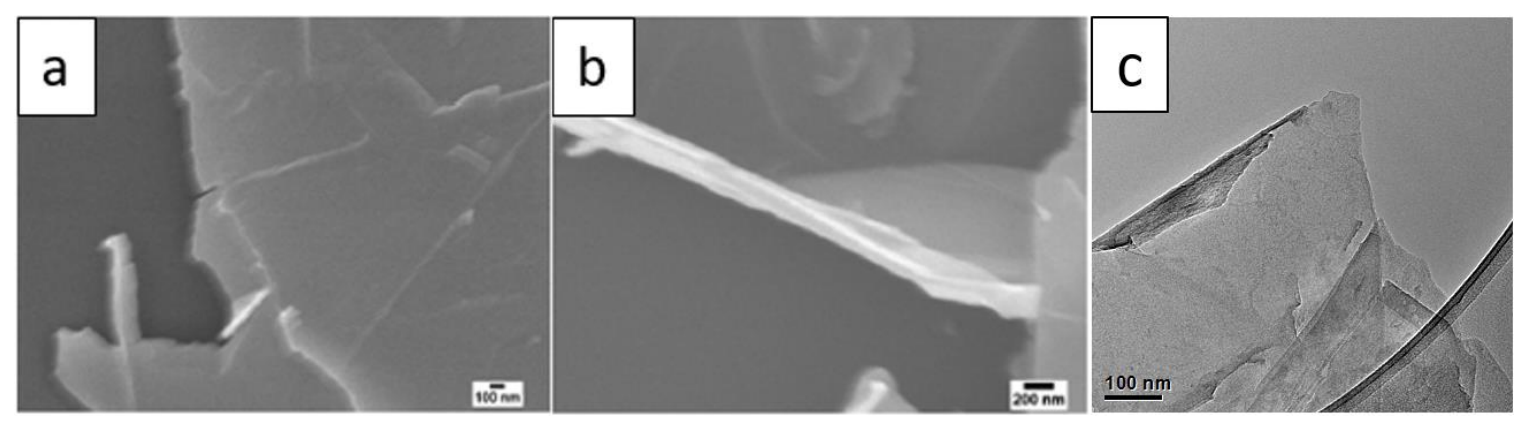

Figure 3.1. (a-b) SEM images of pure GNP powder showing its (a) top surface and (b) a cross section image showing a stack of few GNPs. (c) TEM image of pure GNP powder.

\subsubsection{Cementation Reaction Materials}

Copper (II) sulfate anhydrous powder with $\geq 99.99 \%$ purity, glacial acetic acid with $\geq 99 \%$ purity, and magnesium powder with $\geq 99 \%$ purity, were procured from SigmaAldrich, St. Louis, MO, USA. The zinc and magnesium ribbon used in this work were obtained from Roto Metals, San Leandro, CA, USA. The solid zinc sheet was $99.9 \%$ pure and had dimensions of 8 " 11 " 10.2 . The $99.8 \%$ pure magnesium ribbon coils were 3.2 mm wide, $0.3 \mathrm{~mm}$ thick, and approximately 20 meters long in total. The magnesium rods were $99.95 \%$ pure, $6.5 \mathrm{~mm} \times 152 \mathrm{~mm}$, and obtained from Solution Materials, LLC, IN, USA.

\subsection{Metallic Particle Deposition via Cementation Process}

\subsubsection{Dispersion of Nanoplatelets}

To obtain effective metallic deposition throughout the GNP surface requires reducing their agglomeration, which is achieved through sonication methods. The initial step to effectively disperse GNP is via tip sonication performed using a Vibra-Cell VCX750 ultrasonic processor (Sonics \& Materials, Inc., Newtown, CT, USA) with a 3/4" 
high gain probe. The sonication medium chosen was acetone since no reactions are believed to occur between GNP and acetone [4]. Larger batches of GNP powder ( $\sim 0.5 \mathrm{~g})$ were sonicated in a $600 \mathrm{~mL}$ beaker using $450 \mathrm{~mL}$ of acetone at $70 \%$ amplitude for 30 minutes. The high energy imparted into the solution from the sonication process leads to a temperature increase. Thus, the beaker was surrounded by an ice bath in order to sufficiently cool the mixture to below the boiling point of acetone, $56^{\circ} \mathrm{C}$.

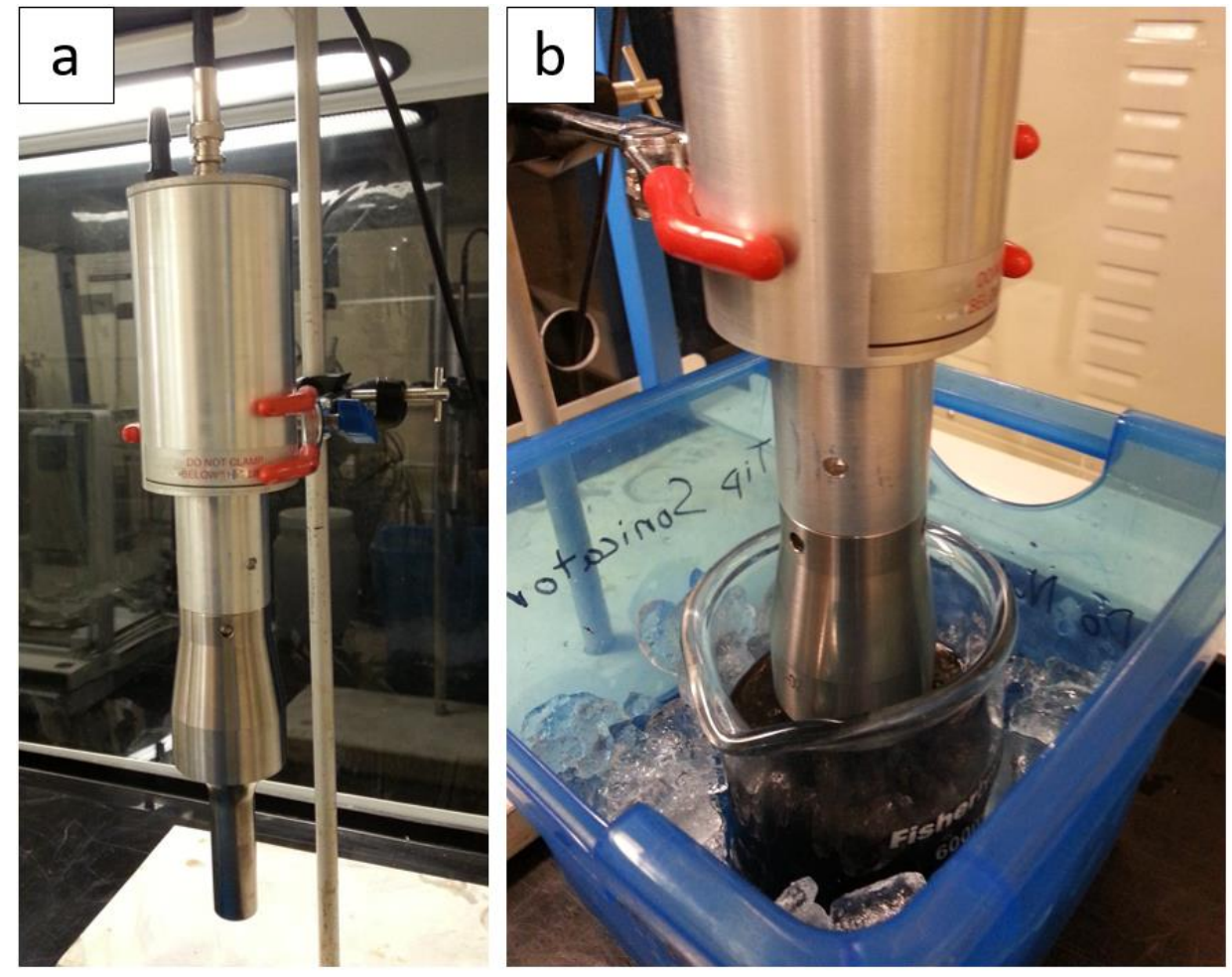

Figure 3.2. (a) Vibra-Cell VCX750 ultrasonic processor equipped with a $3 / 4$ " high gain probe. (b) Tip-sonicating GNP in acetone medium.

Following tip sonication, the GNP powders were fully dried overnight in an oven at $70-80^{\circ} \mathrm{C}$ in order to accelerate the evaporation of acetone without adverse effects on the GNP. To prepare for the cementation synthesis, the powders were then broken up by mortar and pestle and weighed. After the desired amount of GNP was weighed for synthesis, this 
powder was transferred to a $600 \mathrm{~mL}$ beaker with $50 \mathrm{~mL}$ of deionized (DI) water. Since the cementation reaction medium is water and GNP agglomerates in this medium, further dispersion of GNP in DI water was carried out via using a Branson 2510 ultrasonic processor (Loveland, CO, USA) for 30 minutes.

\subsubsection{Cementation Reaction Synthesis}

For an efficient cementation reaction synthesis, the subsequent steps should be followed:

i. Preparation of Metal Reducer Surface

ii. Preparation of $\mathrm{CuSO}_{4}$ Salt Solution and Surface Activation of GNP

iii. Start of Reaction via Introduction of Reducer

iv. $\quad$ Filtering and Washing

The next sections will discuss the listed cementation reaction steps in detail.

\subsubsection{Preparation of Metal Reducer Surface}

For an efficient cementation reaction process with minimal impurities, preparation of a metal reducer is necessary. Metal reducers used in this work are zinc and magnesium, chosen due to their higher reactivity when compared to copper. Different forms of reducers were used due to their differing surface area. The zinc sheets purchased were cut into strips and pieces, while the magnesium reducer forms explored were rod, powder, and ribbon. This was achieved by polishing the metal reducer surfaces using SiC paper in order to obtain a pure and unoxidized metal surface. All forms of $\mathrm{Zn}$ and $\mathrm{Mg}$ were able to be polished except for magnesium powder. To clean the particles left by the polishing process, the metal reducers were then wiped with lint free wipes and acetone. Figure 3.3(a,b) shows 
images of zinc strips and magnesium ribbons used, while Figure 3.3(c) illustrates the difference between polished and unpolished Mg ribbon.
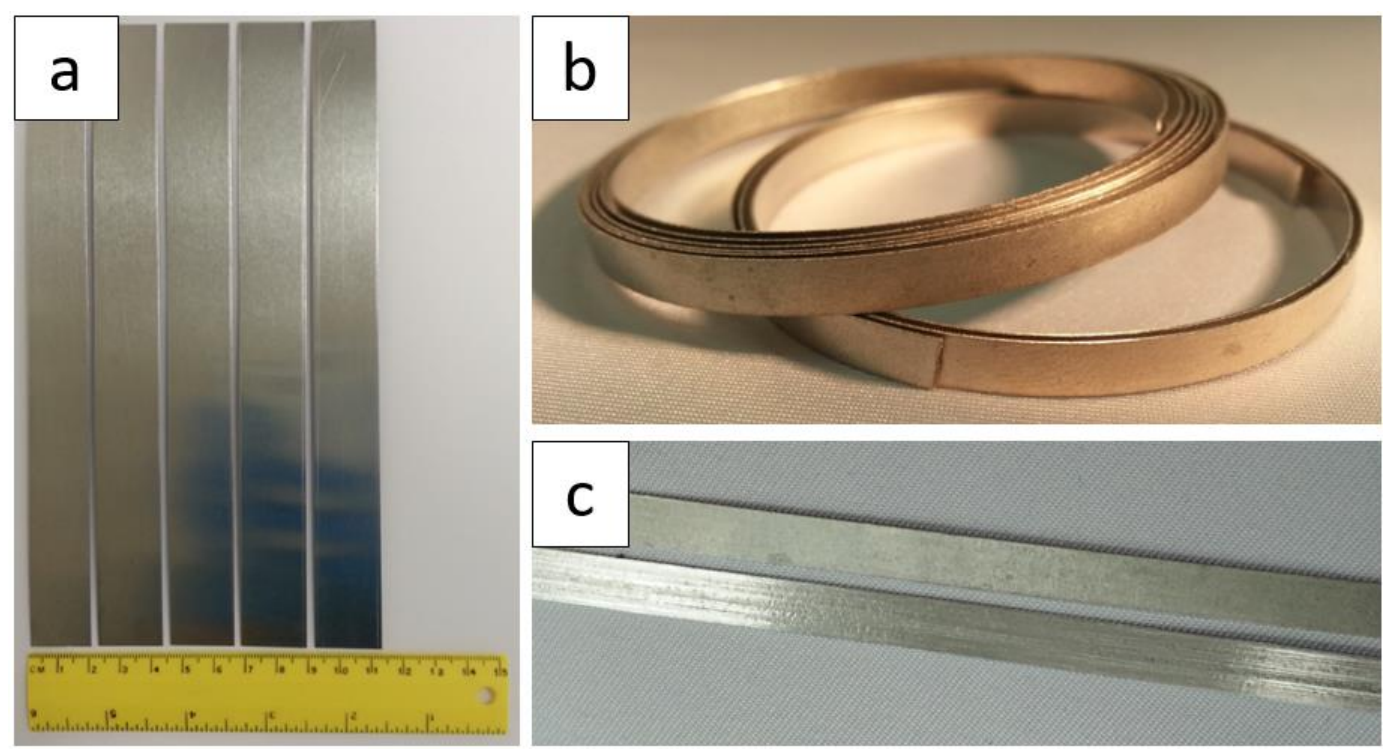

Figure 3.3. (a) As purchased zinc sheet cut into $2 \mathrm{~cm}$ strips. (b) As-purchased magnesium ribbon coils. (c) Magnesium strip before (top) and after polishing (bottom)

\subsubsection{Preparation of $\mathrm{CuSO}_{4}$ Salt Solution and Surface Activation of GNP}

The copper(II) sulfate salt was weighed depending on the concentration desired and added to a $250 \mathrm{~mL}$ beaker with $50 \mathrm{~mL}$ of deionized water. This salt solution was mixed with a magnetic stirrer until the salt was fully dissolved and a light blue aqueous solution was obtained, as can be seen in Figure 3.4a. At this point, the surface activator, glacial acetic was added to this $\mathrm{CuSO}_{4}$ aqueous solution and stirred for 3 minutes to ensure the liquids were fully mixed. This blue $50 \mathrm{~mL}$ aqueous solution of $\mathrm{CuSO}_{4}$ and acetic acid was subsequently added to the dispersed GNP in $50 \mathrm{~mL}$ of water. This is the surface activation step, where the surface of GNP is "activated" by the acetic acid in solution. This occurs by allowing the GNP to be stirred (by magnetic stirrer) into the acetic acid solution for a set amount of time. 

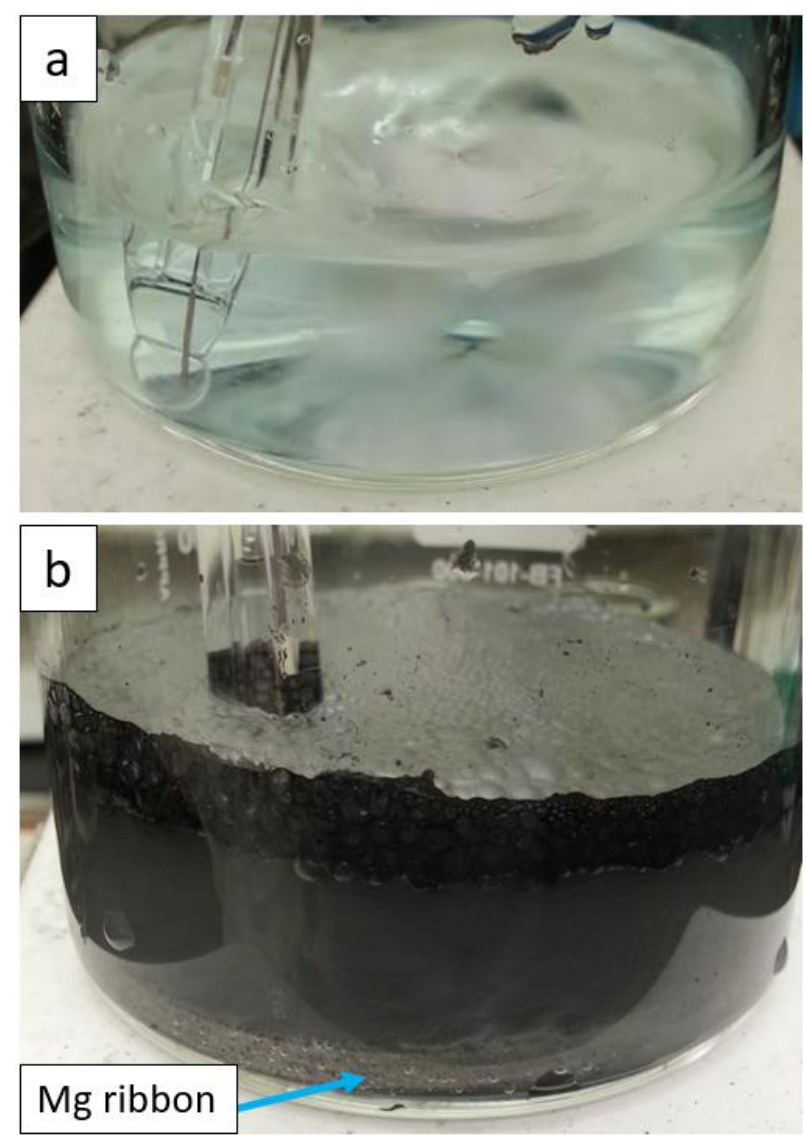

Figure 3.4. (a) Slightly blue hue of $0.01 \mathrm{M}$ copper (II) sulfate aqueous solution continuously stirred by magnetic stirrer. (b) A picture during cementation reaction. A piece of the magnesium ribbon can be observed at the bottom while the solution is continuously stirred.

\subsubsection{Start of Reaction via Introduction of Reducer}

The reaction begins by inserting the polished and clean metal reducer into the solution with the activated GNP, while the solution was continuously stirred by the magnetic stirrer, as can be seen in Figure 3.4b. The $\mathrm{pH}$ of the solution was monitored using a Hanna Instruments HI 2210 pH meter (Woonsocket, RI, USA).

\subsubsection{Filtering and Washing}

The reaction was allowed to continue for a determined amount of time. The GNP 
with copper precipitates $(\mathrm{GNP}-\mathrm{Cu})$ were separated from the aqueous solution via gravimetric filtering using a $125 \mathrm{~mm}$ diameter Whatman (Maidstone, UK) filter paper with $2.5 \mu \mathrm{m}$ pore size. The filter paper was folded and placed in a glass funnel. The gravimetric filtering setup can be seen in Figure 3.5b. After the GNP-Cu powder was fully filtered from solution, the powder was washed multiple times with DI water in order to diminish any impurities present, in particular $\mathrm{MgSO}_{4}$ (aq) that was formed during the reaction. The GNP-Cu powder was allowed to dry in the filter overnight (Figure 3.5c and 3.6).
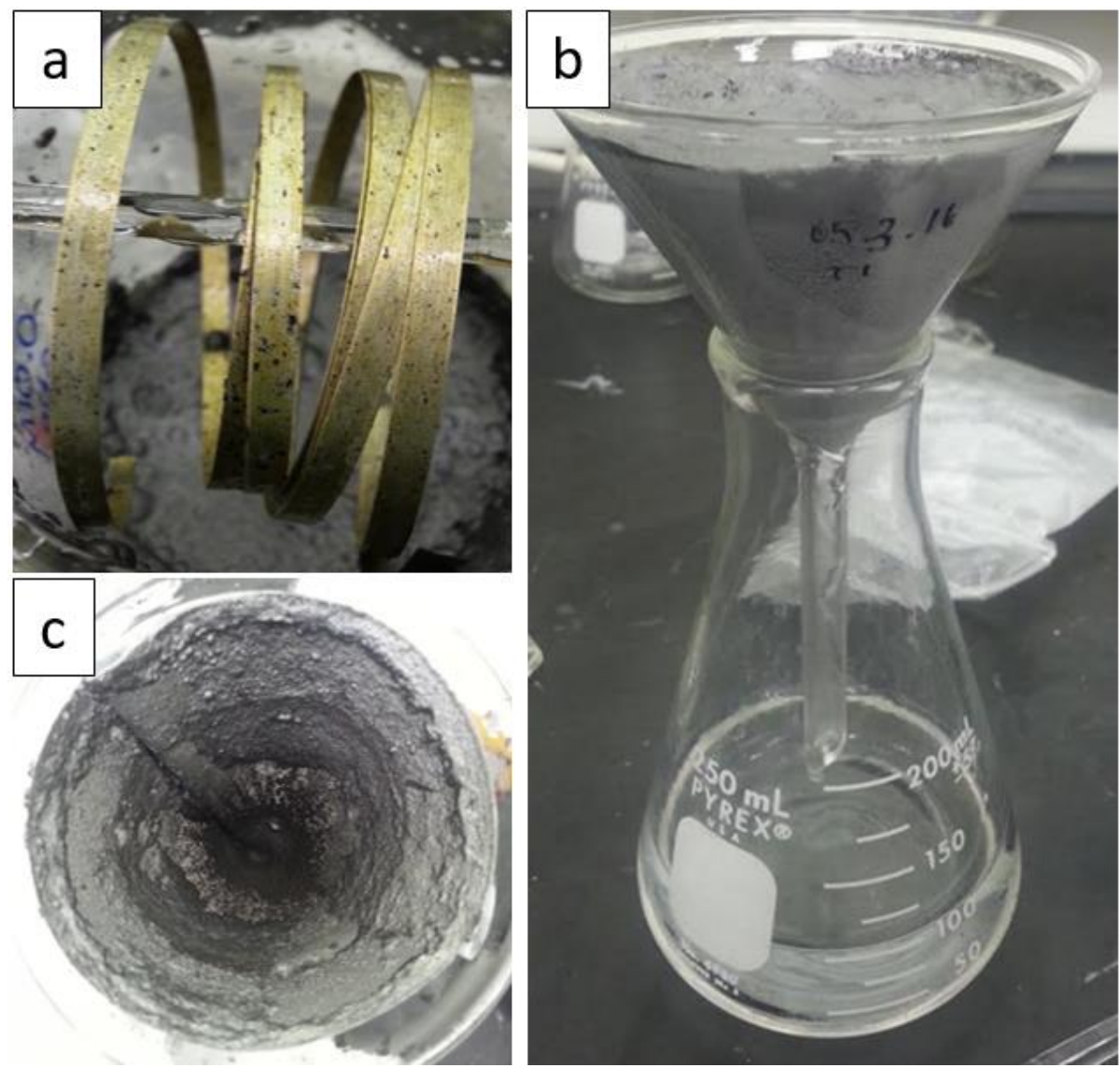

Figure 3.5. (a) Magnesium ribbon reducer after the reaction ends. (b) Gravimetric filtering setup: filter paper is placed inside glass funnel and filtrate is released into Erlenmeyer flask. (c) Wet GNP-Cu powder inside filter paper left overnight to dry. 


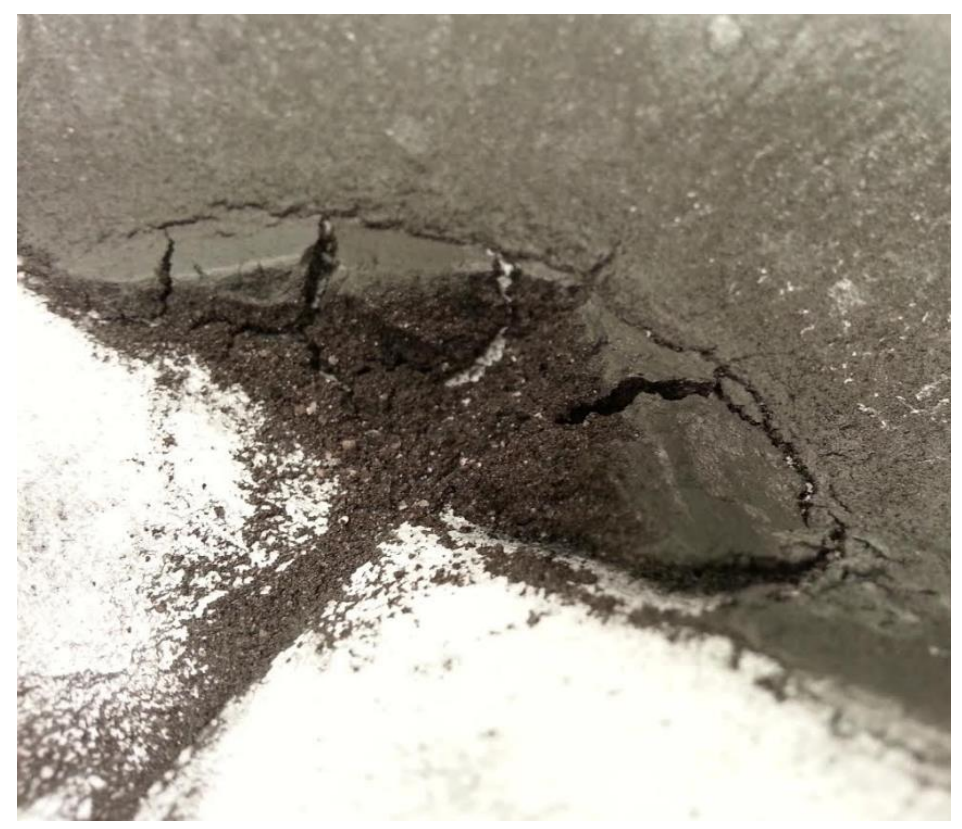

Figure 3.6. Fully dried GNP-Cu powder on the filter paper.

\subsection{Parametric Studies}

Optimization of parameters used in cementation reactions was studied in this work in order to obtain:

- Uniform deposition of copper particles on GNP surface with minimal clustering

- Low copper concentration (approximately 5 vol.\%)

- Nanometer size copper particles

- Minimum impurities

The parameters considered to reach the specific goals outlined above are:

I. Metal reducer

II. Acetic Acid

III. Copper(II) sulfate concentration 
Table 3.1 outlines the parametric studies carried out in this work using metal reducers (studied type, form and concentration), acetic acid (surface activation process and varying volume \%) and different copper (II) sulfate concentrations.

Table 3.1. Parametric studies of metal reducers, acetic acid and copper (II) sulfate concentration to achieve a uniform deposition of nanometer-sized $\mathrm{Cu}$ particles on GNP with minimum impurities

\begin{tabular}{|c|c|c|c|c|}
\hline \multicolumn{5}{|c|}{ Metal Reducer } \\
\hline \multicolumn{3}{|c|}{ Magnesium } & \multicolumn{2}{|c|}{ Zinc } \\
\hline Rod & Powder & Ribbon & Strip & Pieces \\
\hline \multicolumn{3}{|c|}{ Mg Ribbon Concentration } & \multicolumn{2}{|c|}{ Zn concentration } \\
\hline 0.66 moles & 0.33 moles & 0.11 moles & Unable to calculate & 0.66 moles \\
\hline \multicolumn{5}{|c|}{ Acetic Acid } \\
\hline \multicolumn{3}{|c|}{ Surface Activation } & \multicolumn{2}{|c|}{ Volume \% } \\
\hline $1 \mathrm{~min}$ & $3 \mathrm{~min}$ & $10 \mathrm{~min}$ & 1 vol.\% & 2 vol. $\%$ \\
\hline \multicolumn{5}{|c|}{ Copper(II) Sulfate Concentration } \\
\hline $0.100 \mathrm{~N}$ & & $50 \mathrm{M}$ & $0.010 \mathrm{M}$ & $0.050 \mathrm{M}$ \\
\hline
\end{tabular}

As shown in Table 3.1, different metal reducers were chosen to be investigated, $\mathrm{Mg}$ and $\mathrm{Zn}$. Compared to $\mathrm{Zn}, \mathrm{Mg}$ is more likely to more effectively reduce copper ions due to being a more reactive metal than $\mathrm{Zn}$, as per the activity series of metals. Varying $\mathrm{CuSO}_{4}$ concentrations were used in order to investigate the effectiveness of $\mathrm{Mg}$ and $\mathrm{Zn}$ as reducers at varying concentrations, as shown in Table 3.2.

Table 3.2. Studies of $\mathrm{Mg}$ ribbon and $\mathrm{Zn}$ pieces in order to discern most effective reducer at varying $\mathrm{CuSO}_{4}$ concentrations 
Compared to $\mathrm{Zn}, \mathrm{Mg}$ ribbon was able to more effectively reduce copper ions, including at lower concentrations of $0.05 \mathrm{M} \mathrm{CuSO}_{4}$. Once $\mathrm{Mg}$ ribbon was found to be the optimal reducer for this study, Table 3.3 details the parametric studies investigated using $\mathrm{Mg}$ ribbon as a reducer, which include: $\mathrm{Mg}$ ribbon concentration, acetic acid (surface activation time and vol.\%), and $\mathrm{CuSO}_{4}$ concentration.

Table 3.3. Parametric studies of $\mathrm{Mg}$ ribbon concentration, acetic acid surface activation process and varying volume percentages, and $\mathrm{CuSO}_{4}$ concentration carried out using $\mathrm{Mg}$ ribbon as the reducer

\begin{tabular}{|c|c|c|c|}
\hline $\mathrm{CuSO}_{4}$ Conc. & $\begin{array}{l}\text { Mg Ribbon } \\
\text { Conc. }\end{array}$ & $\begin{array}{l}\text { Acetic } \\
\text { Acid }\end{array}$ & $\begin{array}{c}\text { Surface } \\
\text { Activation }\end{array}$ \\
\hline $0.100 \mathrm{M}$ & 0.66 moles & 2 vol. $\%$ & $1 \mathrm{~min}$ \\
\hline $0.100 \mathrm{M}$ & 0.66 moles & 2 vol. $\%$ & $3 \mathrm{~min}$ \\
\hline $0.050 \mathrm{M}$ & 0.66 moles & 2 vol. $\%$ & $3 \mathrm{~min}$ \\
\hline $0.050 \mathrm{M}$ & 0.33 moles & 2 vol. $\%$ & $3 \min$ \\
\hline $0.050 \mathrm{M}$ & 0.11 moles & 2 vol. $\%$ & $3 \mathrm{~min}$ \\
\hline $0.050 \mathrm{M}$ & 0.33 moles & 1 vol. $\%$ & $3 \min$ \\
\hline $0.010 \mathrm{M}$ & 0.33 moles & 1 vol. $\%$ & $3 \mathrm{~min}$ \\
\hline $0.005 \mathrm{M}$ & 0.33 moles & 1 vol. $\%$ & $3 \mathrm{~min}$ \\
\hline
\end{tabular}

\subsection{Characterization of GNP-Cu Powders}

\subsubsection{Microstructural Characterization and Chemical Composition}

True density of the synthesized GNP-Cu powder was investigated using Accupyc 1340 Helium gas pycnometer from Micromeretics Instrument Corporation (Norcross, GA, USA). Phase components were analyzed by means of X-Ray Diffraction (XRD) using Cu K $\alpha$ radiation (wavelength of $1.542 \AA$ ). The instrument used was a Bruker D5000 X-ray 
diffractometer (Billerica, MA, USA). The operating voltage and current used were $40 \mathrm{kV}$ and $35 \mathrm{~mA}$ respectively with a scan rate of $2 \%$ minute. Scanning electron microscopy was carried out using a JEOL JSM-6330 field emission scanning electron microscope (JEOL USA, Inc., Peabody, MA, USA) with an operating voltage of $15 \mathrm{kV}$. Equipped with the SEM is an energy dispersive X-ray spectrometer (EDS) which was used to identify the components of synthesized samples as well as any impurities that may be present. Transmission electron microscopy (TEM) was carried out using a FEI/Philips CM-200T (Hillsboro, OR, USA). ImageJ software was used to determine the approximate size of the copper deposits on the surface of the GNP. Similar characterization was carried out for sintered GNP-Cu compact. The apparent bulk density measurements of the compacts were carried out using a water immersion technique based on the Archimedes Principle. 


\section{CHAPTER IV}

\section{Results and Discussion}

This chapter examines synthesis and characterization results and the underlying science. The specific goal is obtain a uniform deposition of copper nanoparticles onto the GNP surface of low copper concentration $(\sim 5$ vol.\%) with minimum clustering and impurities. The experimental parameters studied and optimized to reach the goals are the type and form of metallic reducers, activation period for GNP dispersion, acetic acid and copper (II) sulfate concentration. The optimal condition for each parameter is presented. Lastly, synthesized GNP-Cu powder using optimal conditions were consolidated via SPS. Though the prime aim of this work is to synthesize and characterize GNP-Cu precursor powder, preliminary characterization of sintered compacts were carried out as well.

\subsection{Influence of Input Parameters on Outcome}

The specific goals of the parametric studies conducted were to obtain:

- Uniform deposition of copper particles on the GNP surface with minimal clustering

- Low copper concentration (approximately 5 vol. \%)

- Nanometer size copper particles

- Minimum impurities present (copper(I) oxide and magnesium hydroxide)

The parameters considered to reach the specific goals outlined above are acetic acid, copper(II) sulfate concentration, and metal reducer. Figure 4.1 illustrates how the input parameters influence the specific goals of this work. The blue sections highlight the optimum condition found. 


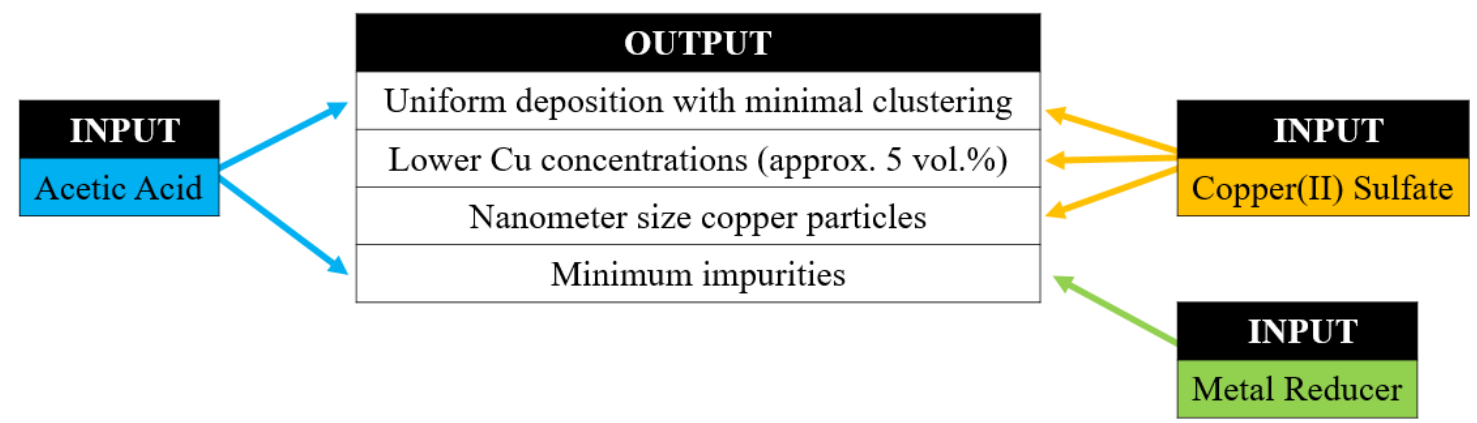

Figure 4.1. Influence of input parameters on the desired final goals of this study.

\subsubsection{Influence of Metallic Reducer}

The metallic reducer used in cementation reactions greatly affects the purity of copper precipitates by controlling the formation of copper oxide and $\mathrm{Mg}$ impurities. In this work, the metallic reducer was investigated in the following ways:

a) Type: Determining most efficient reducer ( $\mathrm{Mg}$ vs $\mathrm{Zn})$

b) Form: Determining most efficient form of $\mathrm{Mg}$

c) Concentration: Determining most efficient concentration of $\mathrm{Mg}$ as reducer

The three ways metal reducers were investigated in this study are: by type ( $\mathrm{Mg}$ or $\mathrm{Zn})$, $\mathrm{Mg}$ form (rod, powder and ribbon) and concentration ( $(0.11,0.33$, and 0.66 moles) as portrayed in Table 4.1.

Table 4.1. Parametric studies carried out on metal reducer investigating type, form and concentration

\begin{tabular}{|c|c|c|c|c|}
\hline \multicolumn{7}{|c|}{ Metal Reducer } \\
\hline \multicolumn{5}{|c|}{ Magnesium } \\
\hline Rod & Powder & Ribbon & Strip & Pieces \\
\hline \multicolumn{2}{|c|}{ Mg Ribbon Concentration } & Zn concentration \\
\hline 0.66 moles & 0.33 moles & 0.11 moles & Unable to calculate & 0.66 moles \\
\hline
\end{tabular}




\subsubsection{Type: Determining Most Efficient Reducer (Mg vs Zn)}

To effectively displace pure copper $(\mathrm{Cu})$ particles from $\mathrm{CuSO}_{4}$ solution to be deposited on GNP's surface, magnesium $(\mathrm{Mg})$ and zinc $(\mathrm{Zn})$ reducers were investigated to determine the most efficient reducer for this work. Due to their relative positions in the standard EMF (Electromotive Force Series) series, both Mg and $\mathrm{Zn}$ metals may act as reducers of copper ions. The standard reduction potentials of $\mathrm{Mg}$ and $\mathrm{Zn}$ are $+2.37 \mathrm{~V}$ and $+0.76 \mathrm{~V}$ respectively, and are detailed in equations (1) and (2). The reduction of potential of $\mathrm{Mg}$ is more positive than that of $\mathrm{Zn}$, indicating that $\mathrm{Mg}$ is a more effective reducer.

$$
\begin{aligned}
& \text { Reducer: } \quad \operatorname{Mg}_{(\mathrm{s})} \Rightarrow \mathrm{Mg}^{2+}{ }_{(\mathrm{aq})}+2 \mathrm{e}^{-} \quad\left(\varepsilon^{\circ}=+2.37 \mathrm{~V}\right) \\
& \text { Reducer: } \quad \mathrm{Zn}_{(\mathrm{s})} \Rightarrow \mathrm{Zn}^{2+}{ }_{(\mathrm{aq})}+2 \mathrm{e}^{-} \quad\left(\varepsilon^{\circ}=+0.76 \mathrm{~V}\right)
\end{aligned}
$$

The greater effectiveness of $\mathrm{Mg}$ as a reducer was observed experimentally by observing each reducer's capability at varying $\mathrm{CuSO}_{4}$ concentrations. Table 4.2 details the parameters, results and conclusions derived from experiments using $\mathrm{Mg}$ and $\mathrm{Zn}$ described.

Table 4.2. Effectiveness of $\mathrm{Mg}$ Ribbon and $\mathrm{Zn}$ Pieces as reducers at varying $\mathrm{CuSO}_{4}$

\begin{tabular}{|c|c|c|c|}
\hline \multicolumn{2}{|c|}{ Mg Ribbon (0.66 moles) } & \multicolumn{2}{|c|}{ Zn Pieces (0.66 moles) } \\
\hline $0.10 \mathrm{M} \mathrm{CuSO}_{4}$ & $0.05 \mathrm{M} \mathrm{CuSO}_{4}$ & $0.10 \mathrm{M} \mathrm{CuSO}_{4}$ & $0.05 \mathrm{M} \mathrm{CuSO}_{4}$ \\
\hline \multicolumn{2}{|c|}{$\begin{array}{l}\text { - Precipitates Cu particles } \\
\text { - Reducer is not coated }\end{array}$} & $\begin{array}{l}\text { - Precipitates } \mathrm{Cu} \text { particles } \\
\text { - Reducer is not coated }\end{array}$ & $\begin{array}{l}\text { - Cu completely coats } \mathrm{Zn} \text {. } \\
\text { This is undesirable as the } \mathrm{Cu} \\
\text { coats } \mathrm{Zn} \text { instead of } \\
\text { precipitating and depositing } \\
\text { onto the GNP surface. }\end{array}$ \\
\hline
\end{tabular}
concentrations

These investigations were performed using $\mathrm{CuSO}_{4}$ concentrations set to $0.10 \mathrm{M}$ and $0.05 \mathrm{M}$ and $2 \mathrm{vol} . \%$ of acetic acid. It was found that $\mathrm{Mg}$ was able to precipitate pure copper 
particles at both $\mathrm{CuSO}_{4}$ concentrations, without getting coated itself. Zinc was able to precipitate pure copper in $\mathrm{CuSO}_{4}$ solutions $\geq 0.10 \mathrm{M}$, though its effectiveness diminished as the concentration of $\mathrm{CuSO}_{4}$ was lowered to $0.05 \mathrm{M}$. As can be observed in Figure 4.2, lowering $\mathrm{CuSO}_{4}$ to $0.05 \mathrm{M}$, resulted in complete coating of the zinc reducer only, with no freely precipitated copper particles left to be deposited onto the surface of GNP.
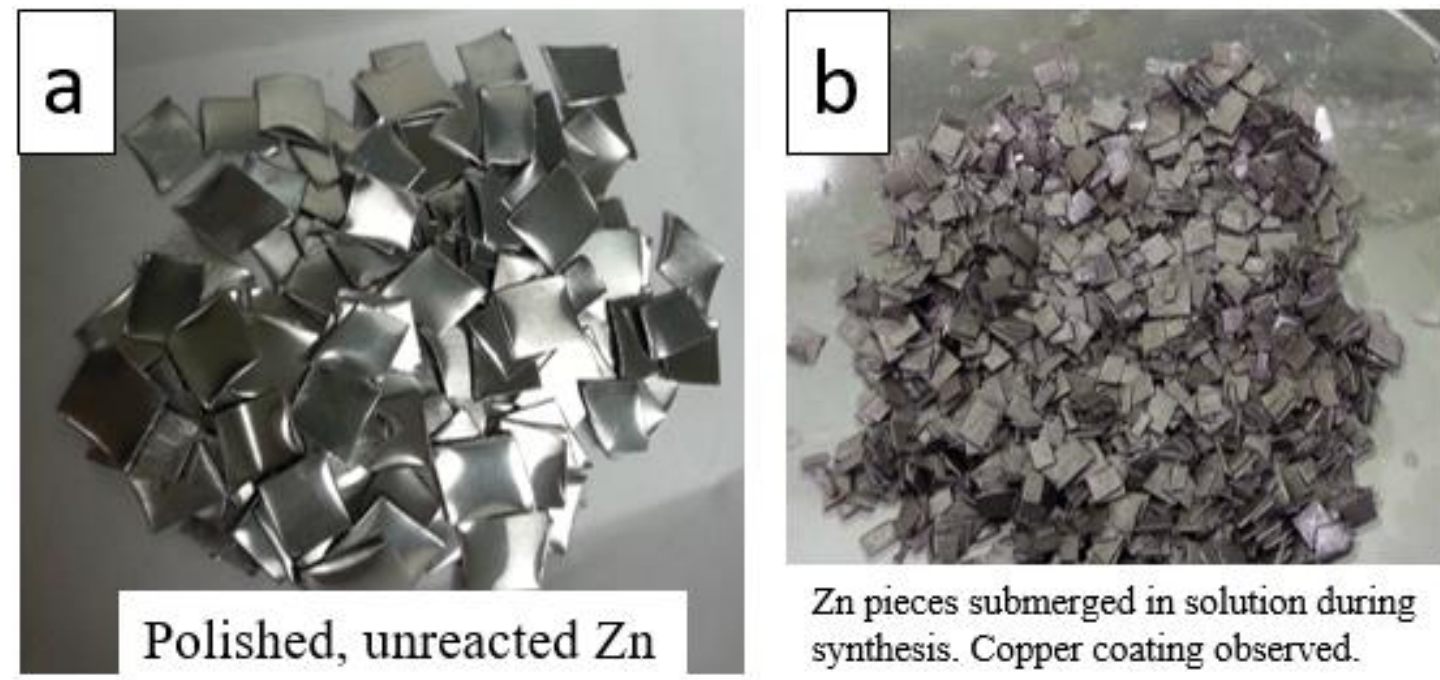

$\mathrm{Zn}$ pieces submerged in solution during synthesis. Copper coating observed.

Figure 4.2. (a) Polished, unreacted surface of $\mathrm{Zn}$ pieces. (c) Post reaction using $0.05 \mathrm{M}$ $\mathrm{CuSO}_{4}$ showing fully copper coated $\mathrm{Zn}$ pieces.

Furthermore, Figure 4.3 and Table 4.3 compares the effectiveness of 0.66 moles of $\mathrm{Mg}$ ribbon and $\mathrm{Zn}$ pieces as reducers via XRD patterns (Fig 4.3) and examining the phase fraction of pure $\mathrm{Cu}$ obtained from the said patterns (Table 4.3). 


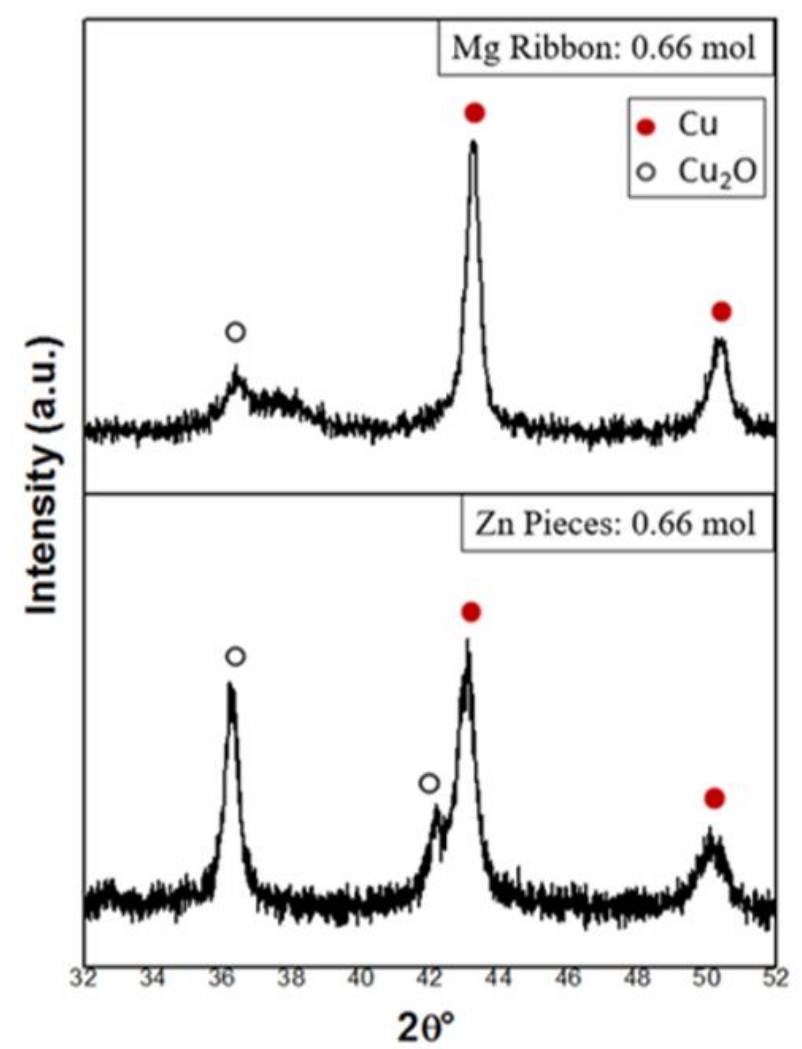

Figure 4.3. XRD patterns showing comparing the effectiveness of 0.66 moles of $\mathrm{Zn}$ pieces and $\mathrm{Mg}$ ribbon in precipitating out pure copper versus copper(I) oxide.

The phase fraction of copper was calculated using the integrated intensity (I) corresponding to cubic copper and cubic copper(I) oxide diffraction peaks as follows:

$$
f_{(\mathrm{Cu})}=\frac{I_{(\mathrm{Cu})}}{I_{(\mathrm{Cu})}+I_{\left(\mathrm{Cu} \mathrm{u}_{2} \mathrm{O}\right.}} \times 100
$$

As can be observed by the increase in pure copper precipitated when using Mg ribbon instead of $\mathrm{Zn},(91.36 \%$ for $\mathrm{Mg}$ versus $52.11 \%$ for $\mathrm{Zn})$ one can conclude that $\mathrm{Mg}$ is a much more effective reducer than $\mathrm{Zn}$. 
Table 4.3. Effectiveness of 0.66 moles of $\mathrm{Zn}$ and $\mathrm{Mg}$ as reducers to precipitate pure coper versus copper(I) oxide

\begin{tabular}{|c|c|c|c|}
$\begin{array}{c}\text { Reducer } \\
\text { Type }\end{array}$ & $\begin{array}{c}\text { Reducer } \\
\text { Quantity }\end{array}$ & Other Parameters & Phase Fraction of Copper \\
\hline Zn pieces & 0.66 moles & CuSO $4=0.10 \mathrm{M}$ & $f_{\mathrm{Cu}}=52.11 \%$ \\
\hline Mg ribbon & 0.66 moles & Acetic Acid $=2$ v. $\%$ & $f_{\mathrm{Cu}}=91.36 \%$ \\
\hline
\end{tabular}

Due to magnesium's superior reducing capabilities when to zinc, for the purposes of this study it was thus determined that Mg ribbon would be the optimal reducer due to its ability to effectively displace $\mathrm{Cu}$ ions from solutions of varying $\mathrm{CuSO}_{4}$ concentrations with lower oxide formation compared to $\mathrm{Zn}$. Also considered is the facile manner in which the oxide layer formed on its surface may be cleaned in order to attain an efficient synthesis.

\subsubsection{Form: Determining Most Efficient Form of Mg}

After determining that $\mathrm{Mg}$ would be the ideal type of reducer for this study, varying forms of $\mathrm{Mg}$ (rod, powder and ribbon) were then investigated. The form of the reducer used changes its surface area availability. This has an impact on the cementation reaction, which takes place at the reducer surface.

Effectiveness of the varying forms of $\mathrm{Mg}$ was performed using the following conditions: $\mathrm{CuSO}_{4}$ concentrations set to $0.10 \mathrm{M}$ and 2 vol.\% of acetic acid. Effectiveness of $\mathrm{Mg}$ forms were explored and their differing effectiveness can be observed in Figure 4.4, which shows the XRD peaks of precipitated copper and copper(I) oxide particles when changing Mg reducer forms. 


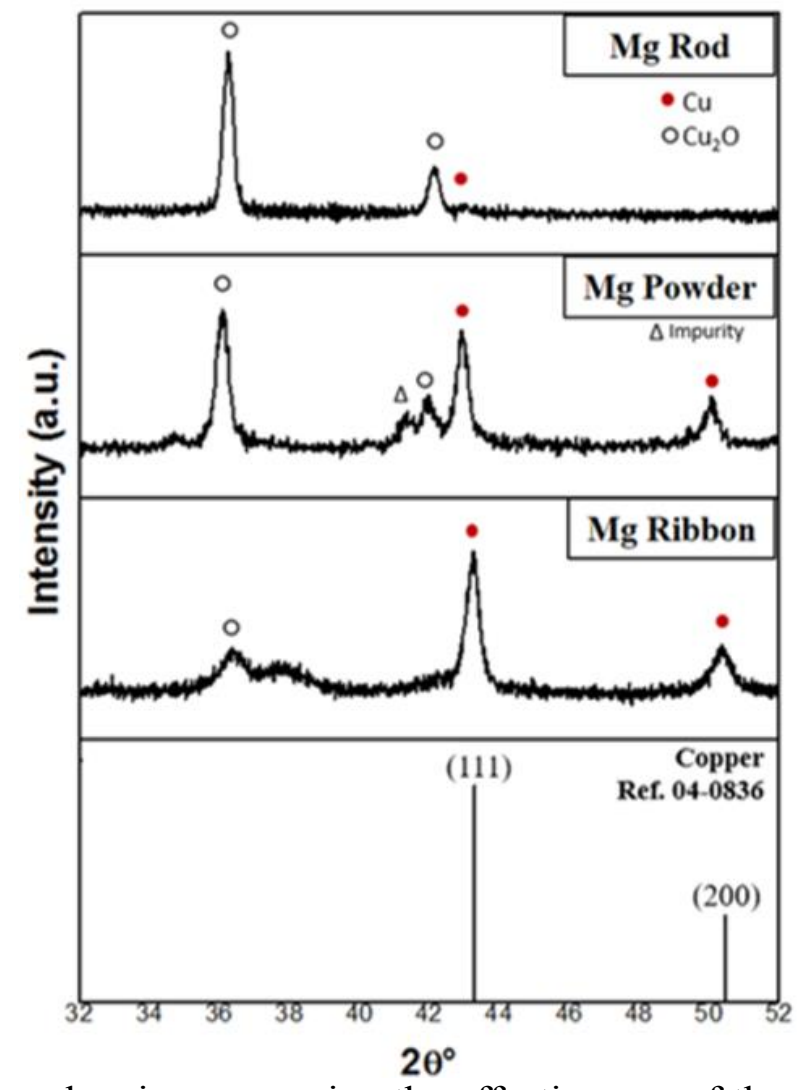

Figure 4.4. XRD patterns showing comparing the effectiveness of the different forms of $\mathrm{Mg}$ (rod, powder, and ribbon) in precipitating pure copper versus copper(I) oxide.

Table 4.4 details the phase fraction of pure $\mathrm{Cu}$ obtained from the said patterns. As shown by Figure 4.4 and Table 4.4, a greatest amount of pure copper is precipitated when using $\mathrm{Mg}$ ribbon, followed by $\mathrm{Mg}$ powder and then $\mathrm{Mg}$ rod. From these results, it was determined that ribbon is the most efficient form of $\mathrm{Mg}$. 
Table 4.4. Effectiveness of different forms of $\mathrm{Mg}$ as reducers in precipitating pure copper versus copper(I) oxide[A1][LF2]

\begin{tabular}{|c|c|c|c|}
\hline \multicolumn{1}{c}{$\begin{array}{c}\text { Reducer } \\
\text { Type }\end{array}$} & $\begin{array}{c}\text { Reducer } \\
\text { Conc. }\end{array}$ & \multicolumn{1}{c|}{ Other Parameters } & Phase Fraction of Copper \\
\cline { 1 - 2 } Mg Rod & $\begin{array}{c}\text { Unable to } \\
\text { calculate }\end{array}$ & \multirow{2}{*}{$\begin{array}{c}\mathrm{CuSO}_{4}=0.10 \mathrm{M} \\
\text { Acetic Acid }=2 \text { vol. } \%\end{array}$} & $f_{\mathrm{Cu}}=3.20 \%$ \\
\cline { 1 - 1 } Mg Powder & 0.66 moles & & $f_{\mathrm{Cu}}=46.94 \%$ \\
\cline { 1 - 1 } Mg Ribbon & 0.66 moles & & $f_{\mathrm{Cu}}=91.36 \%$ \\
\hline
\end{tabular}

Reason as to why this behavior is observed due to the increased metallic surface area present in $\mathrm{Mg}$ ribbon compared to $\mathrm{Mg}$ rod, which leads to more effective $\mathrm{Cu}$ precipitation. Cementation reactions are first order reactions in which the rate controlling step is the diffusion of the metal ions to the metallic surface of the reducer [8]. Though $\mathrm{Mg}$ powder has the greatest surface area and should be the most effective reducing form of $\mathrm{Mg}$, the powder surface is easily oxidized. The oxide layer formed hinders the cementation reaction process where a clean metallic surface is necessary for the reduction of $\mathrm{Cu}$ ions. Most of the oxide layer formed on $\mathrm{Mg}$ ribbon may easily be polished away prior to synthesis in order to obtain a pure metallic surface to eliminate impurities and more effectively reduce copper ions into pure copper.

It was determined that $\mathrm{Mg}$ 's reducing capabilities are most efficient when it is in the form of a ribbon, when compared to rod and powder.

\subsubsection{Concentration: Determining Most Efficient Concentration of Mg as Reducer}

The concentration of reducer used has an effect on the purity of pure copper precipitated, having the ability to minimize the formation of magnesium and oxide 
impurities. Mg-based impurities were detected via XRD and EDS analysis. These impurities were observed in a greater degree in trials using 0.66 moles of $\mathrm{Mg}$ ribbon reducer (Figure 4.5) XRD patterns indicate that the Mg impurities were found to be in the form of magnesium hydroxide, $\operatorname{Mg}(\mathrm{OH})_{2}$, as the impurity peaks aligned with the main peaks of $\mathrm{Mg}(\mathrm{OH})_{2}$ found when $2 \theta$ equals $18.6^{\circ}, 37.9^{\circ}, 50.8^{\circ}$ (overlap with $\mathrm{Cu}$ ) and $58.6^{\circ}$ (PDF Reference Card 04-011-5938).

In order to decrease the presence of $\mathrm{Mg}(\mathrm{OH})_{2}$, the concentration of $\mathrm{Mg}$ ribbon was decreased from 0.66 moles to 0.33 moles, while using $0.05 \mathrm{M} \mathrm{CuSO}_{4}$, and 2 vol. $\%$ of acetic acid. As shown by the XRD patterns in Figure 4.5a, the main magnesium hydroxide peak found at $\sim 37.9^{\circ}$ is nonexistent as the quantity of the $\mathrm{Mg}$ ribbon is reduced from 0.66 moles to 0.33 moles. Decreasing the moles of $\mathrm{Mg}$ ribbon used decreases the $\mathrm{Mg}(\mathrm{OH})_{2}$ impurity due to the oxide layer formed on $\mathrm{Mg}$. Though the $\mathrm{Mg}$ ribbon is polished prior to the synthesis, $\mathrm{Mg}$ is quite reactive thus extremely thin oxide layers of $\mathrm{MgO}$ could still be present on its surface which leads to the formation of $\mathrm{Mg}(\mathrm{OH})_{2}$ when in water. Being able to successfully reduce $\mathrm{Mg}$ impurities is of importance since many studies do not consider $\mathrm{Mg}$ as a reducer for cementation reactions due to its high reactivity which usually leads to high impurity content to be found.

As the concentration of $\mathrm{Mg}$ ribbon is further reduced to 0.11 moles, a decrease in the purity of copper was observed once again, but this time the impurity arose from the greater precipitation of copper(I) oxide. The increase in $\mathrm{Cu}_{2} \mathrm{O}$ formation as reducer quantity decreases is attributed to the lower quantity of metallic reducer available to reduce the copper ions in solutions into pure copper. As further confirmation, this behavior was also observed when using $\mathrm{Zn}$ pieces as a reducer which serves as further confirmation that the 
concentration of reducer used impacts copper(I) oxide formation. Showing that decreasing its quantity by a factor of $\sim 6$ (from 0.66 moles to 0.10 moles of $\mathrm{Zn}$ ) decreased the phase fraction of pure copper from $52.11 \%$ to $10.61 \%$ (Figure $4.5 b$ ).
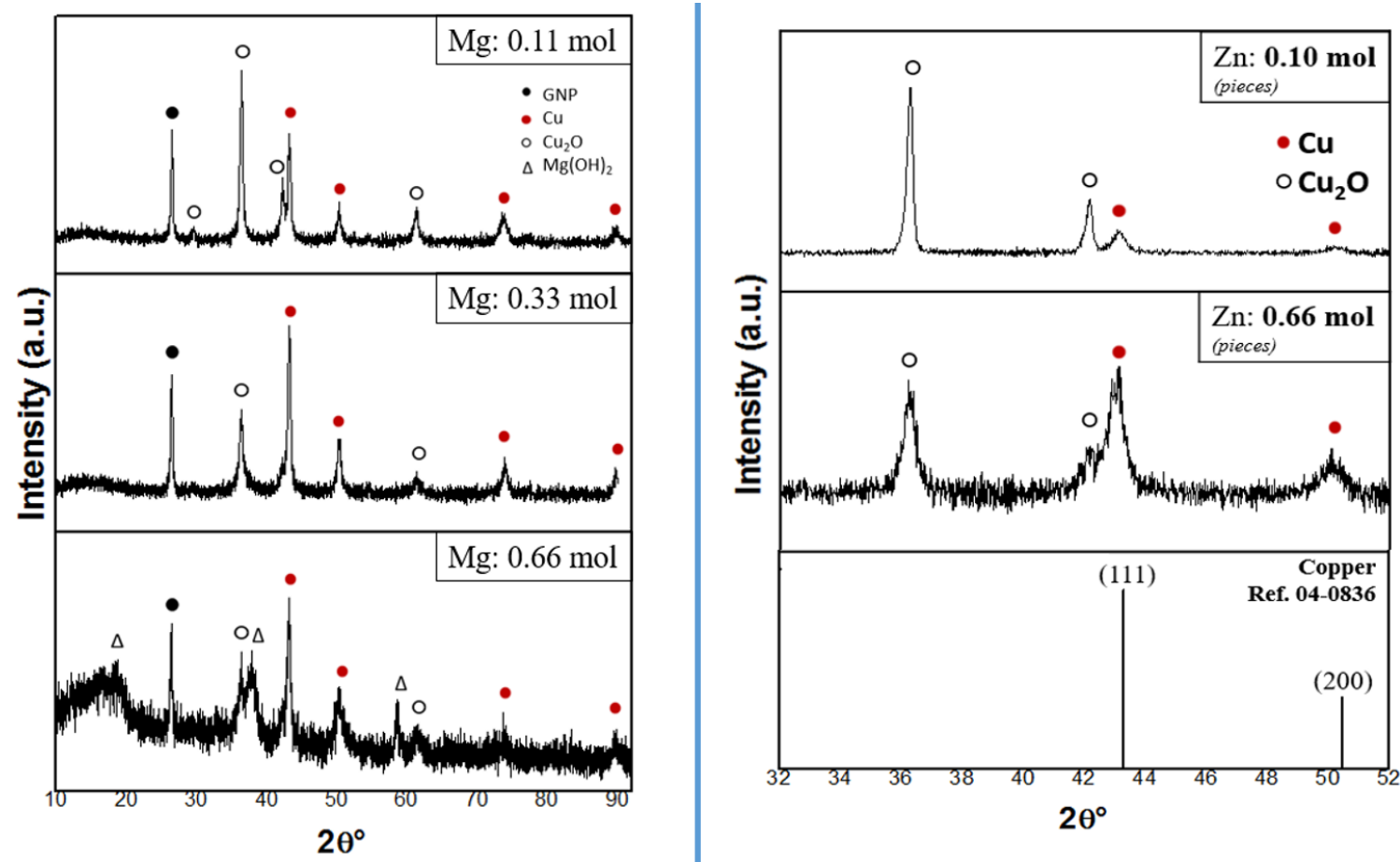

Figure 4.5. (a) XRD patterns showing the reducing effectiveness of $\mathrm{Mg}$ ribbon as a function of reducing moles. (b) Though $\mathrm{Zn}$ was discontinued, XRD patterns showing the reducing effectiveness of $\mathrm{Zn}$ pieces as a function of moles for further confirmation that moles of reducer impacts copper(I) oxide formation.

Figure 4.6 illustrates the purity of copper precipitated as a function of moles of $\mathrm{Mg}$ ribbon used, with $0.05 \mathrm{M} \mathrm{CuSO}_{4}$ and 2 vol.\% acetic acid conditions. When used higher quantity of $\mathrm{Mg}$ ribbon $(0.66 \mathrm{~mol})$, the phase fraction of $\mathrm{Cu}\left(f_{\mathrm{Cu}}\right)$ is high $(74.71 \%)$ compared to $\mathrm{Cu}_{2} \mathrm{O}$, though $\mathrm{Mg}$ impurity is present. As the amount of moles is decreased to $0.33, f_{\mathrm{Cu}}$ remains equally as high (74.32\%) though the $\mathrm{Mg}$ impurity is no longer present. As the amount of moles is further decreased to $0.11 \mathrm{~mol}, f_{\mathrm{Cu}}$ is sharply decreased to $38.61 \%$, though the $\mathrm{Mg}$ impurity is also not present. It was then decided that for these investigations, using 0.33 moles of $\mathrm{Mg}$ ribbon would result in copper precipitates of the highest purity. 


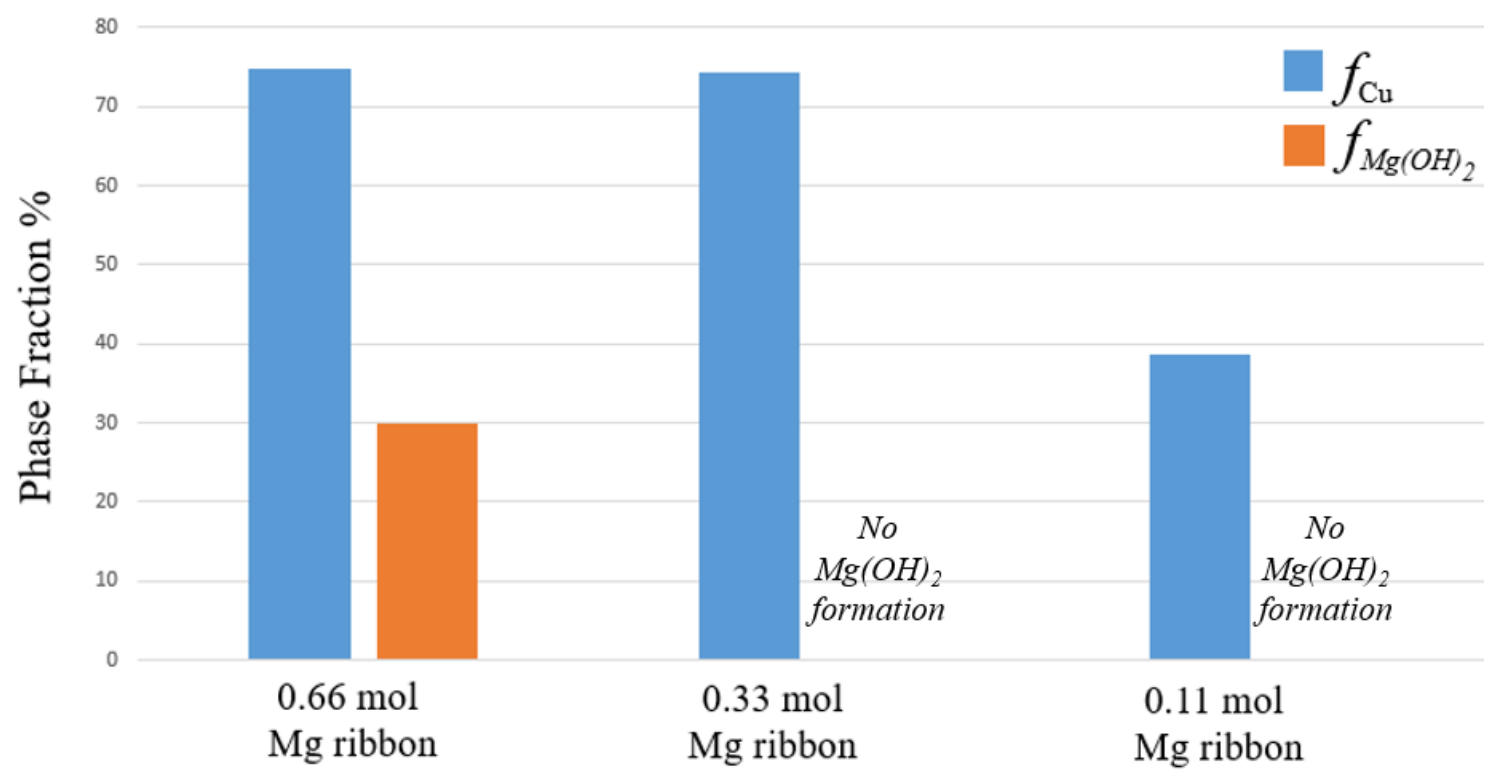

Figure 4.6. Purity of copper precipitated as a function of moles of $\mathrm{Mg}$ ribbon reducer under $0.05 \mathrm{M} \mathrm{CuSO}_{4}$ and 2 vol.\% acetic acid conditions.

\subsubsection{Influence of Acetic Acid as Surface Activator}

Acetic acid influences the specific outcomes of this work in two different ways. First, it plays a role as a surface activator which aids in the metallic deposition onto the GNP surface itself. It also plays an important role in minimizing copper (I) oxide impurities.

\subsubsection{GNP Surface Activation by Acetic Acid}

Before considering the role of acetic acid as a surface activator for uniform deposition, reduction of GNP agglomeration is crucial for uniform deposition to be achieved and will thus be briefly discussed. GNP dispersion was achieved by tipsonication, however cementation reactions occur in aqueous media, which induces GNP agglomeration once more. Due to this, further dispersion techniques were considered. As 
discussed in Chapter 3, to combat this, GNP was ultrasonicated in DI water prior to the cementation process and the reactants were then added to this dispersed GNP beaker. Figure 4.7 illustrates via SEM images the difference in GNP agglomeration in trials without (heavily agglomerated) and with (higher dispersion) ultrasonication prior to the reaction.
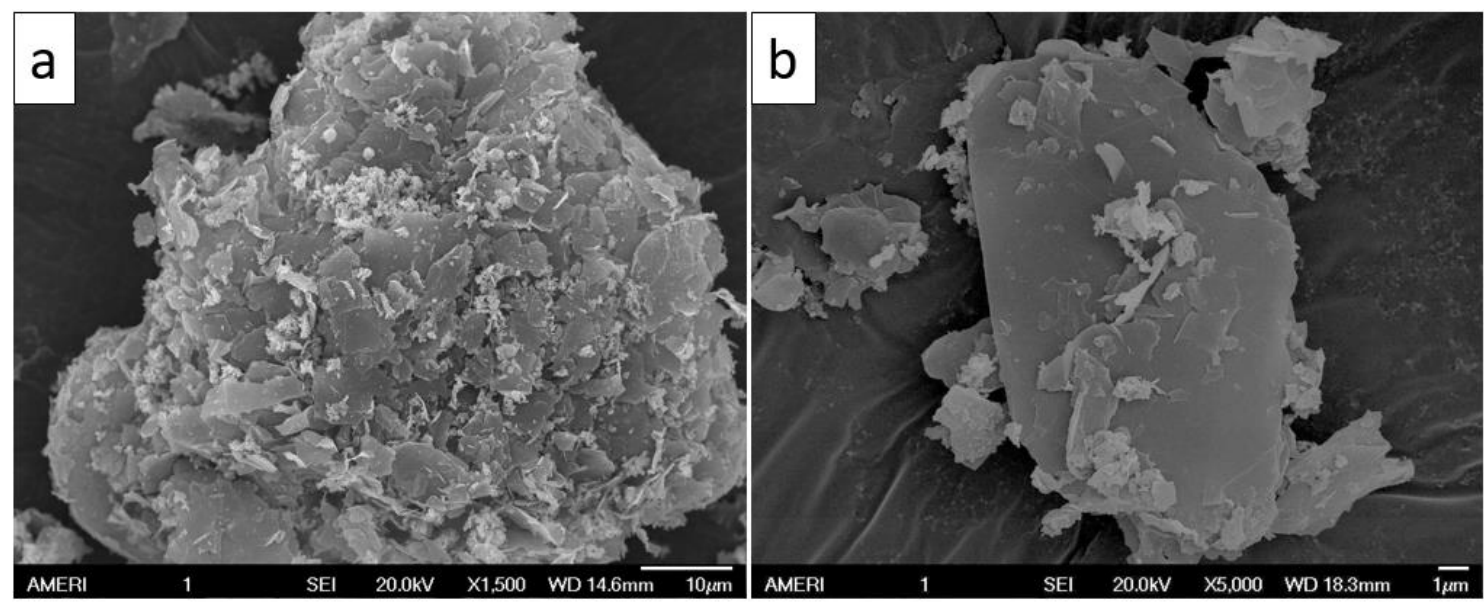

Figure 4.7. (a) GNP-Cu powder without ultrasonication in water prior to cementation process shows heavily agglomerated nanoplatelets. (b) GNP-Cu powder that was ultrasonicated in water prior to cementation process shows further dispersion and fewer nanoplatelets.

After successful dispersion of GNP, its surface activation by acetic acid was investigated. Acetic acid dissociates in water as such:<smiles>CC(=O)OCCCO</smiles>

acetic acid<smiles></smiles>
acetate

The equation above shows that acetic acid will dissociate in water to have a partially negative charge. When allowing the GNP to sit in acetic acid solution, the surface of the GNP becomes "activated" by acetic acid, and gains the partially negative charge from the 
acid, as shown in Figure 4.8.

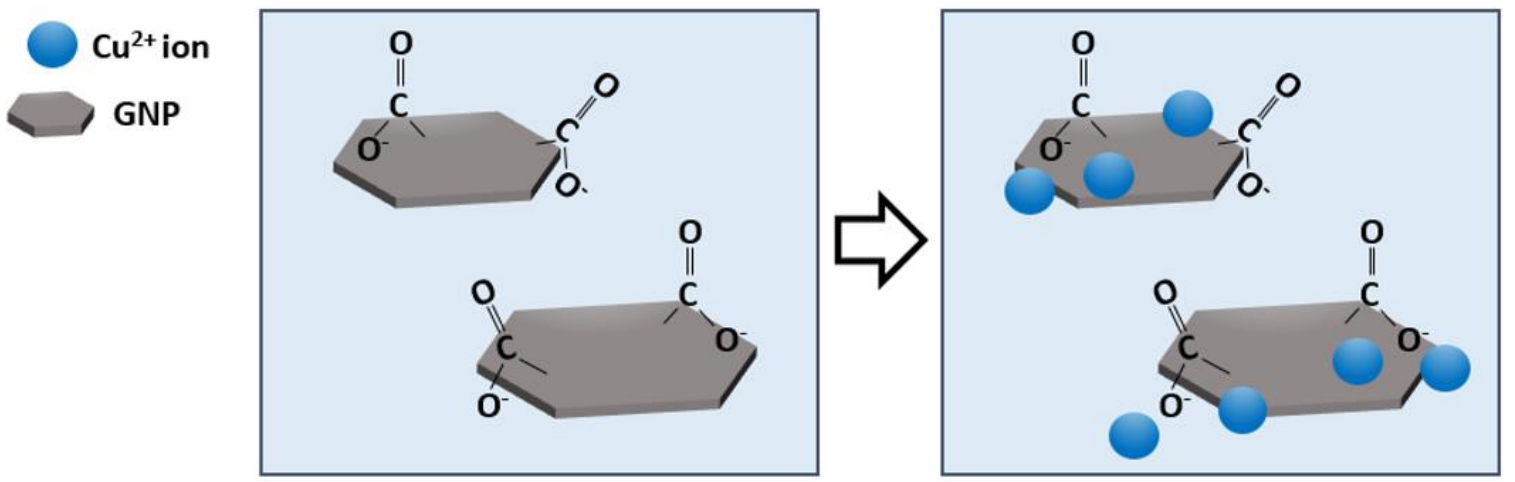

Figure 4.8. Proposed GNP surface activation process by acetic acid.

The positive charge on the $\mathrm{Cu}^{2+}$ ions in solution (from the $\mathrm{CuSO}_{4}$ salt) will be attracted to now partially negative surface of the GNP. When the Mg reducer is inserted signaling the beginning of the cementation reaction, the $\mathrm{Cu}^{2+}$ attracted to the surface of the GNP is then reduced into pure $\mathrm{Cu}_{(\mathrm{s})}$ on the surface of the GNP itself. Thus, increasing the amount of time the GNP surface is allowed to be "activated" by acetic acid leads to greater $\mathrm{Cu}_{(\mathrm{s})}$ particle deposition on the GNP surface itself, as is demonstrated by Figure 4.8.

The GNP surface must be activated by acetic acid for metallic deposition onto its surface to occur, thus the optimal amount of time for this process to be carried out was then investigated. Figure 4.9(a) shows an SEM image of GNP that was allowed to be activated for 1 minute. From this condition, freely precipitated copper particles are observed around the GNP, but no copper particles are observed on the GNP surface itself. Increasing the surface activation process to 3 minutes shows copper particles deposited on the GNP surface, as can be seen in Figure 4.9 (b,c).

Both experiments were carried out with $0.10 \mathrm{M} \mathrm{CuSO}_{4}, 0.66$ moles of $\mathrm{Mg}$ ribbon, and 2 vol.\% of glacial acetic acid, the surface activator. To further study this parameter, 
the surface activation process was then increased to 10 minutes. This resulted in GNP-Cu powder that was "fluffy", and extremely difficult to handle for analysis. The density of pure GNP is $1.82 \mathrm{~g} / \mathrm{cm}^{3}$, while that of acetic acid is $1.05 \mathrm{~g} / \mathrm{cm}^{3}$ [4]. Allowing the GNP to be activated by acetic acid for 10 min resulted in a GNP-Cu powder that appeared to be of lower density. Due to the extreme difficulty in handling this powder, this approach was discontinued and surface activation time of 3 min was chosen instead. 


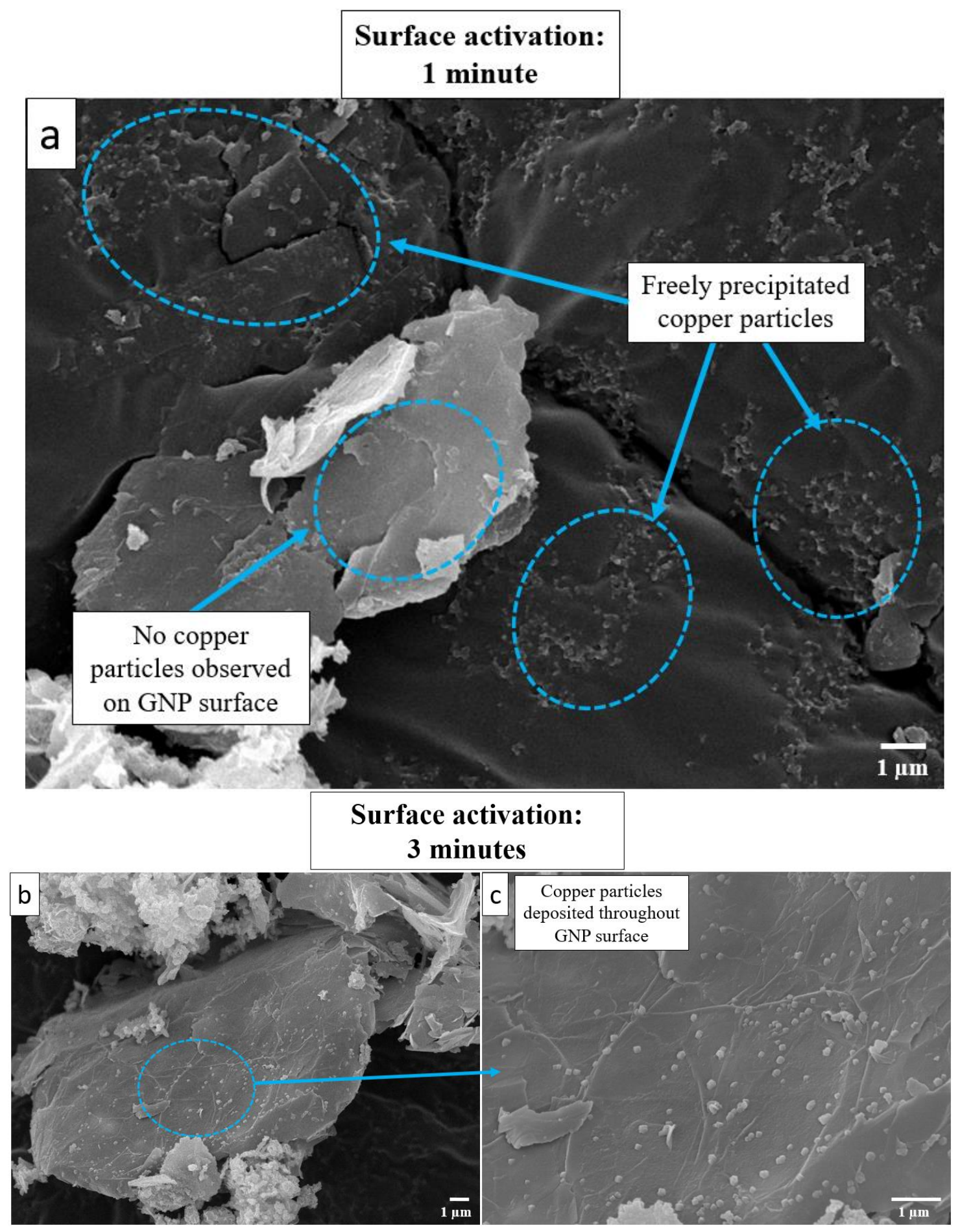

Figure 4.9. (a) GNP surface activated for 1 minute shows freely $\mathrm{Cu}$ precipitates, with no particles on GNP surface. (b,c) Increasing the surface activation to 3 minutes resulted in $\mathrm{Cu}$ particles deposited onto the GNP surface. 


\subsubsection{Varying Concentration of Acetic Acid to Minimize Impurities}

Volume percentage of acetic acid was varied in order to further investigate the way this parameter influences the specific aims of this work, to obtain a uniform deposition of $\mathrm{Cu}$ nanoparticles on the surface of GNP with minimum impurities present. In reactions using $0.05 \mathrm{M} \mathrm{CuSO}_{4}$ and 0.33 moles of $\mathrm{Mg}$ ribbon, it appears that decreasing the amount of acetic acid from 2 vol. $\%$ to 1 vol. $\%$ allows the copper to be precipitated more effectively by minimizing copper(I) oxide formation. This is observed from the increase in the phase quantification of copper $\left(f_{\mathrm{Cu}}\right)$, compared to $\mathrm{Cu}_{2} \mathrm{O}$, which is increased from $74.3 \%$ to $81.6 \%$, (Figure 4.10).

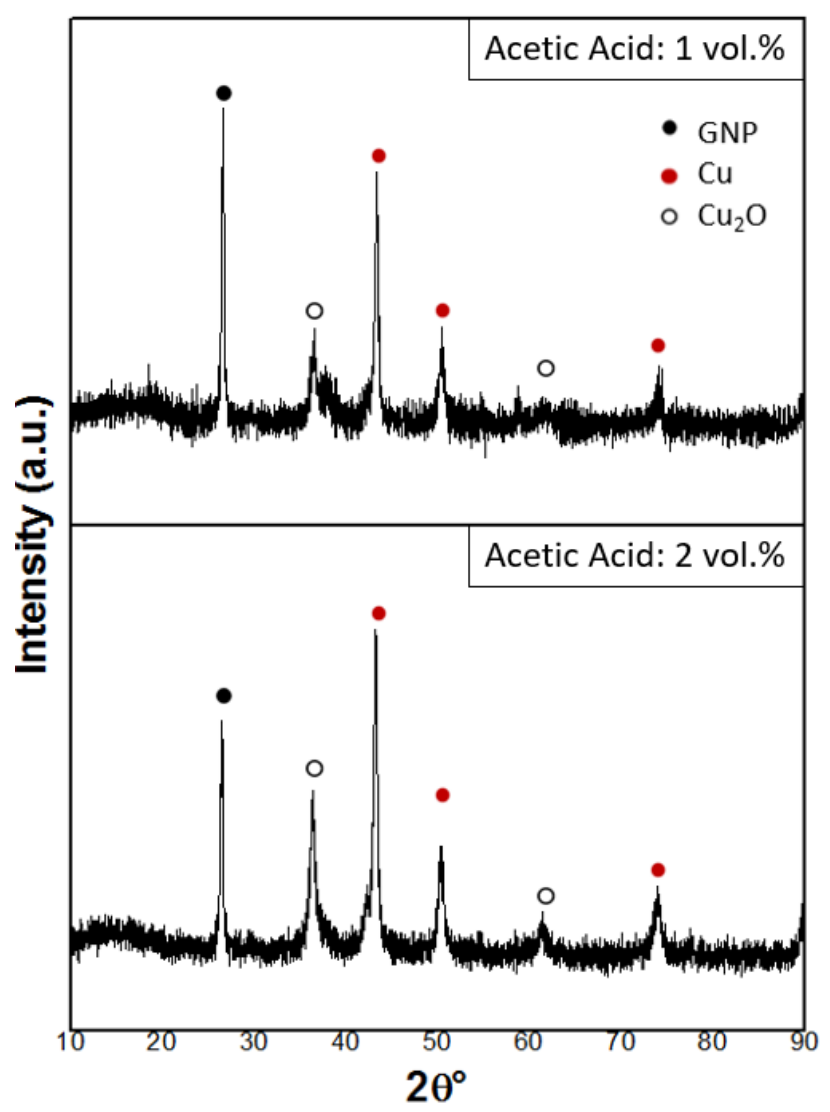

Figure 4.10. XRD patterns of GNP-Cu powder synthesized from $0.05 \mathrm{M} \mathrm{CuSO}_{4}$ solutions varying the amount of acetic acid used from 2 vol.\% to 1 vol. $\%$, resulting in more effective $\mathrm{Cu}$ precipitation when compared to $\mathrm{Cu}_{2} \mathrm{O}$. 
The increase in $f_{\mathrm{Cu}}$ observed when acid vol.\% is decreased may be attributed to the fact that the $\mathrm{Mg}$ ribbon surface will be more readily corroded in solutions containing higher vol.\% of acid. The acetic acid will readily corrode the surface of the Mg ribbon, resulting in $\mathrm{H}_{2(\mathrm{~g})}$ formation at the metallic surface, as per the reaction:

$$
\mathrm{Mg}_{(\mathrm{s})}+\mathrm{CH}_{3} \mathrm{COOH}_{(\mathrm{aq})} \rightarrow \mathrm{MgCH}_{3} \mathrm{COOH}_{(\mathrm{aq})}+\mathbf{H}_{2} \text { (g) }
$$

Since cementation reactions are dependent upon the diffusion of the metal ions to the metallic surface of the reducer, the $\mathrm{H}_{2}$ gas formed by the acidic corrosion could inhibit the reduction of $\mathrm{Cu}$ ions into pure $\mathrm{Cu}$ from occurring at the reducer surface. The $\mathrm{Cu}$ ions could then be partially reduced to $\mathrm{Cu}^{1+}$, resulting in $\mathrm{Cu}_{2} \mathrm{O}$ formation due to the reaction occurring in an open beaker. It is expected that a greater concentration of partially reduced $\mathrm{Cu}^{1+}$ ions are found closer to the reducer surface. Possible mechanism of this process is illustrated in Figure 4.11.

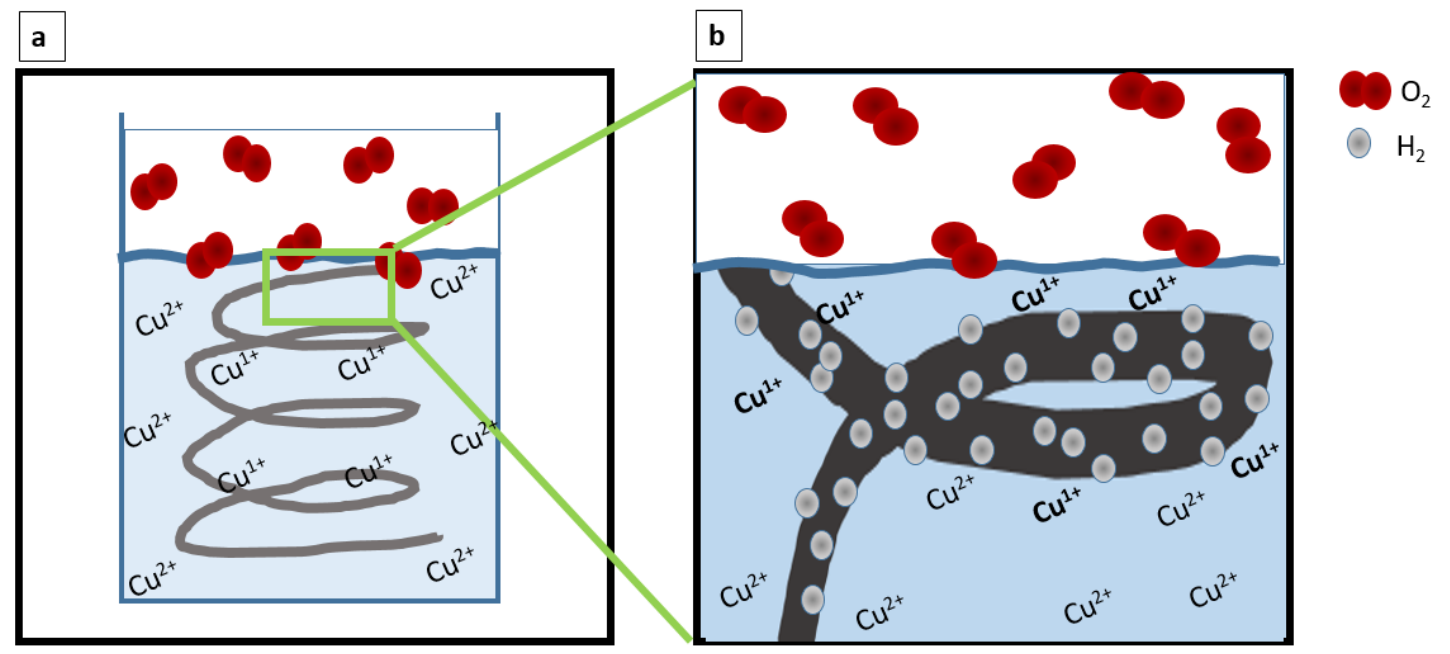

Figure 4.11. Possible mechanism showing $\mathrm{H}_{2}$ gas evolution from $\mathrm{Mg}$ ribbon surface due to acetic acid, inhibiting cementation process and resulting in $\mathrm{Cu}_{2} \mathrm{O}$ formation. 
It was determined that acetic acid impacts the specific goals of this work by acting as a surface activator, aiding in the uniform deposition of $\mathrm{Cu}$ onto the surface of GNP. Optimum surface activation conditions were achieved by allowing the process to occur for 3 minutes. Furthermore, the volume $\%$ of acetic acid used impacts the formation of copper(I) oxide impurities. The optimum amount of acetic acid for minimum impurity formation was found to be 1 volume $\%$. The optimum conditions of acetic acid determined in this work are highlighted in Table 4.5.

Table 4.5. Parametric investigations of surface activation process and volume $\%$ using acetic acid with optimum conditions highlighted in blue

\begin{tabular}{|c|c|c|c|c|}
\hline \multicolumn{3}{|c|}{ Acetic Acid } \\
\hline \multicolumn{3}{|c|}{ Surface Activation } & \multicolumn{3}{c|}{ Volume \% } \\
\hline $1 \mathrm{~min}$ & $3 \mathrm{~min}$ & $10 \mathrm{~min}$ & $1 \mathrm{vol} \%$ & 2 vol.\% \\
\hline
\end{tabular}

\subsubsection{Influence of Copper (II) Sulfate}

The concentration of copper (II) sulfate is a significant parameter which affects (i) the uniformity of metallic deposition on the GNP surface with minimum clustering, (ii) achieving low copper concentrations of approximately 5 vol.\%, and (iii) nanometer size copper particles.

Initially, to confirm that the particles deposited onto the GNP surface as observed, by SEM and TEM images (Figure 4.12 (a-d)), were indeed copper particles, X-ray diffraction and EDS analysis were employed (Figures 4.12 (f-g)). Analysis of GNP-Cu powder synthesized using $0.01 \mathrm{M} \mathrm{CuSO}_{4}, 0.33 \mathrm{~mol}$ of $\mathrm{Mg}$ ribbon, and 1 vol.\% of acetic acid is discussed. Formation of copper is confirmed by XRD analysis, where the main copper peaks are found at $2 \theta$ values of about $43.3^{\circ}, 50.4^{\circ}, 74.1^{\circ}$, and $89.9^{\circ}$ (PDF Reference 
Card 00-004-0836) (Figure 4.12f). Further confirmation of the formation of copper is obtained by EDS data (Figure 4.12g). EDS indicates that the sample is $77.73 \mathrm{wt} . \% \mathrm{C}$ from GNP and $18.28 \mathrm{wt} . \% \mathrm{Cu}$. Also found is the presence of Mg as impurity, though in the very low amount of $0.28 \mathrm{wt} . \%$. Also of note is the selected area diffraction (SAD) pattern which indicates that the particles on the GNP surface are crystalline and most importantly, in the nanometer scale.

The analytical methods (SEM. TEM, XRD, and EDS) were employed to ascertain that the synthesis method studied resulted in the precipitation of pure copper nanoparticles onto the GNP surface, with minimum impurities. 

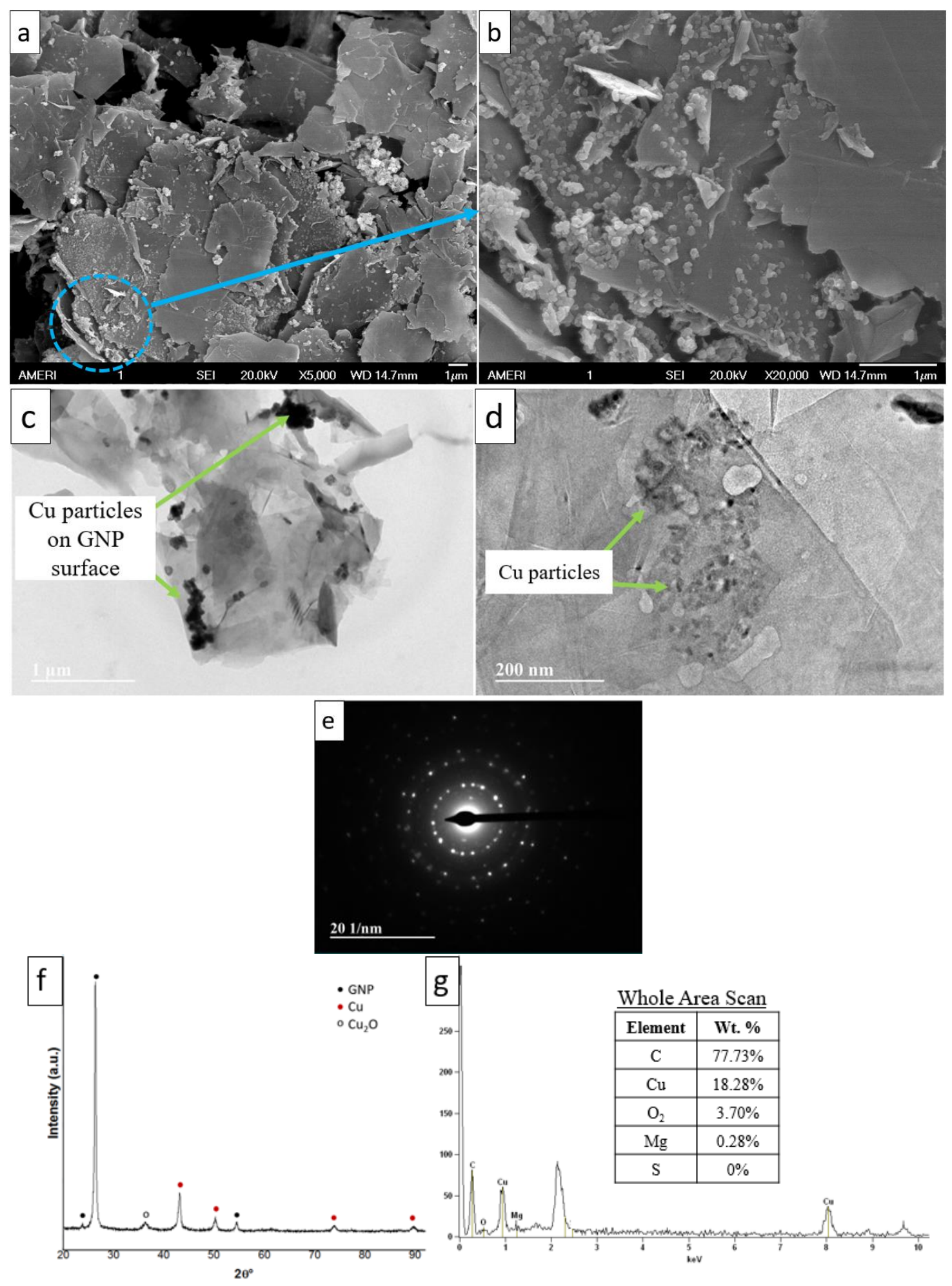

Figure 4.12. (a,b) SEM images showing Cu nanoparticles on the GNP surface. (c,d) TEM images showing $\mathrm{Cu}$ nanoparticles on the GNP surface. (e) SAD pattern of $\mathrm{Cu}$ nanoparticles (f) XRD pattern of this trial shows the main peaks of $\mathrm{Cu}$, indicating $\mathrm{Cu}$ nanoparticles were precipitated. (g) Whole area EDS indicates the presence of $\mathrm{Cu}$, and minimum impurities. 
For this study, the copper (II) sulfate concentration was varied from $0.10 \mathrm{M}, 0.05$ M, 0.01 M and 0.005 M, as shown in Table 4.6.

Table 4.6. Varying concentrations of Copper (II) Sulfate solution investigated in this study

\begin{tabular}{|c|c|c|c|}
\hline \multicolumn{4}{|c|}{ Copper(II) Sulfate Concentration } \\
\hline $0.100 \mathrm{M}$ & $0.050 \mathrm{M}$ & $0.010 \mathrm{M}$ & $0.050 \mathrm{M}$ \\
\hline
\end{tabular}

At higher copper concentrations of $0.10 \mathrm{M}$ and $0.05 \mathrm{M}$, SEM images show that though copper particles were deposited onto the GNP surface (after allowing GNP's surface to be activated by acetic acid for 3 minutes), copper clusters were precipitated around the GNP as well. Decreasing the copper concentration leads to less copper precipitation and thus diminishes the frequency of copper clusters found around GNP, as shown in Figure 4.13(a-d). Copper clusters are unwanted in this investigation, which aims to obtain a very thin and uniform layer of copper particles deposited onto the GNP surface itself. 


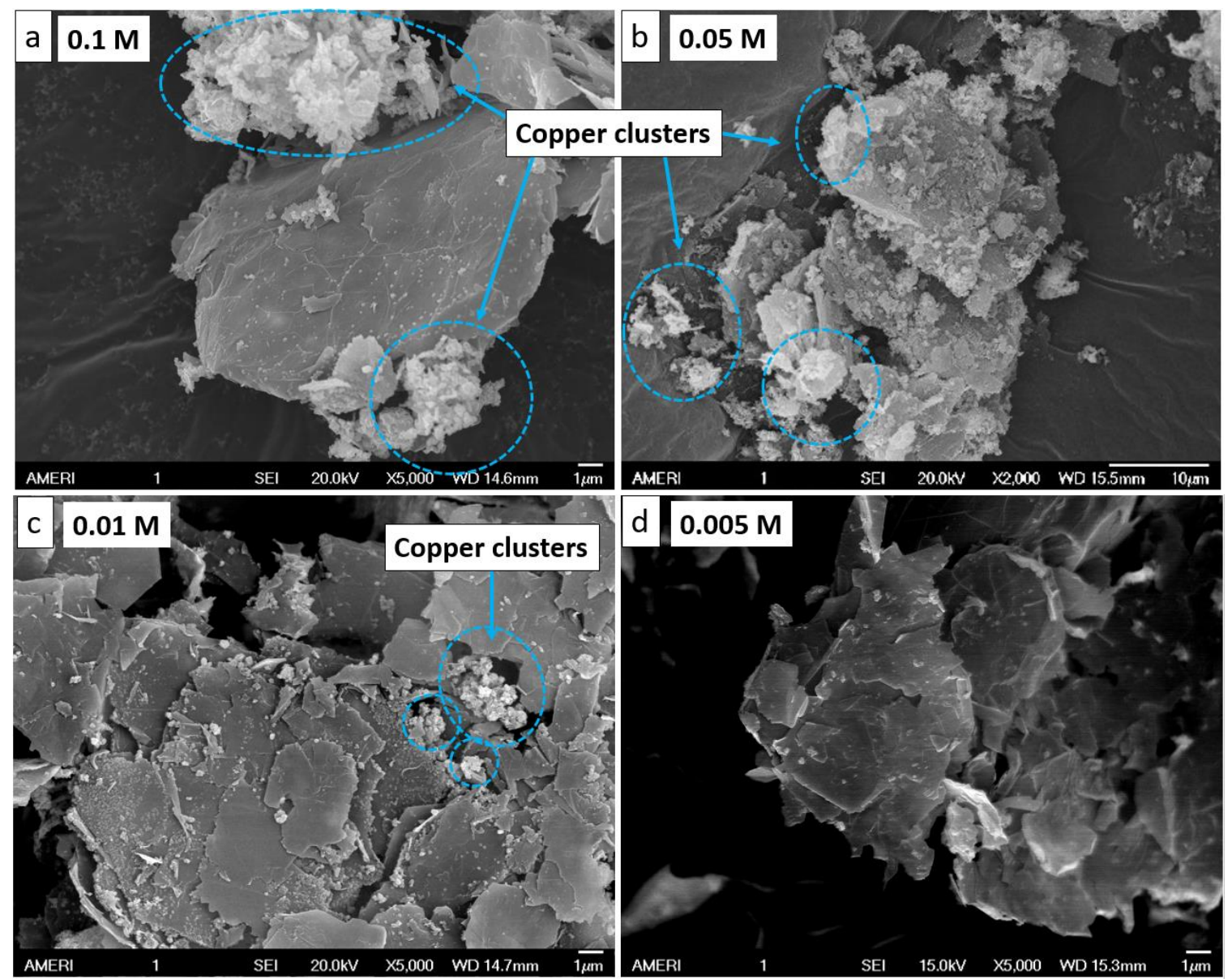

Figure 4.13. SEM images reveal that the frequency of precipitated copper clusters around GNP diminishes as $\mathrm{CuSO}_{4}$ concentration decreases from $0.10 \mathrm{M}$ to $0.005 \mathrm{M}$ (a-d).

It was also observed that decreasing the copper concentration also leads to smaller copper particle size and crystallite size (Table 4.7). Further observation of the particle and crystallite sizes indicates that the copper particles precipitated are polycrystalline in nature. Greater $\mathrm{CuSO}_{4}$ concentration lead to larger particle sizes due to the larger amount of $\mathrm{Cu}$ ions becoming reduced at the metallic reducer interface into a growing copper deposit. Interestingly, for samples synthesized from $0.10 \mathrm{M}$ and $0.05 \mathrm{M} \mathrm{CuSO}_{4}$ solutions, the trend does not hold true since the amount of acetic acid was changed from 2 vol.\% to 1 vol.\%. From these trials (Trials 1 and 2 in Table 4.7) one can observe how the increased acetic 
acid amount leads to smaller crystallite sizes. This may be attributed to the increased $\mathrm{H}_{2(\mathrm{~g})}$ formation at increased vol. $\%$ of acid. The increased gas formation on the metallic reducer surface could be impeding the nucleation and precipitation process of the crystallites, leading to smaller crystallite sizes when compared to solutions with lesser vol.\% of acid.

Table 4.7. Decreasing particle and crystallite size of precipitated copper particles as a function of decreasing $\mathrm{CuSO}_{4}$ concentration and acetic acid volume \%

\begin{tabular}{|c|l|c|c|c|c|}
\hline Trial & \multicolumn{1}{l}{ Concentration } & \multicolumn{1}{c}{ Mg Ribbon } & \multicolumn{1}{c|}{ Acetic Acid } & \multicolumn{1}{c|}{ Particle Size } & Crystallite Size \\
\hline $\mathbf{1}$ & $\mathbf{0 . 1 0 0 ~ M}$ & $0.99 \mathrm{~mol}$ & $2 \mathrm{vol} \%$ & $\begin{array}{c}75.34 \pm \\
14.15 \mathrm{~nm}\end{array}$ & $\begin{array}{c}21.8 \pm \\
9.2 \mathrm{~nm}\end{array}$ \\
& & & & & \\
\hline $\mathbf{2}$ & $\mathbf{0 . 0 5 0} \mathbf{M}$ & $0.33 \mathrm{~mol}$ & 1 vol. \% & $63.48 \pm$ & $22.4 \pm$ \\
& & & & $19.94 \mathrm{~nm}$ & $9.2 \mathrm{~nm}$ \\
\hline $\mathbf{3}$ & $\mathbf{0 . 0 1 0 ~ M}$ & $0.33 \mathrm{~mol}$ & 1 vol. \% & $54.32 \pm$ & $19.1 \pm$ \\
& & & & $14.35 \mathrm{~nm}$ & $5.1 \mathrm{~nm}$ \\
\hline $\mathbf{4}$ & $\mathbf{0 . 0 0 5} \mathbf{M}$ & $0.33 \mathrm{~mol}$ & 1 vol. \% & $48.33 \pm$ & $11.9 \mathrm{~nm}$ \\
& & & & $14.30 \mathrm{~nm}$ & \\
\hline
\end{tabular}


Table 4.8 details the parametric investigations carried out on the metallic reducer, acetic acid and copper(II) sulfate concentration in order to optimize synthesis conditions to obtain the specific goals of this work. The blue sections highlight the optimum condition found.

Table 4.8. Parametric studies of metal reducers, acetic acid and copper(II) sulfate concentration with highlighted optimum conditions to achieve a uniform deposition of nanometer-sized $\mathrm{Cu}$ particles on GNP with minimum impurities

\begin{tabular}{|c|c|c|c|c|}
\hline \multicolumn{5}{|c|}{ Metal Reducer } \\
\hline \multicolumn{3}{|c|}{ Magnesium } & \multicolumn{2}{|c|}{ Zinc } \\
\hline Rod & Powder & Ribbon & Strip & Pieces \\
\hline \multicolumn{3}{|c|}{ Mg Ribbon Concentration } & \multicolumn{2}{|c|}{ Zn concentration } \\
\hline 0.66 moles & 0.33 moles & 0.11 moles & Unable to calculate & 0.66 moles \\
\hline \multicolumn{5}{|c|}{ Acetic Acid } \\
\hline \multicolumn{3}{|c|}{ Surface Activation } & \multicolumn{2}{|c|}{ Volume \% } \\
\hline $1 \mathrm{~min}$ & $3 \mathrm{~min}$ & $10 \mathrm{~min}$ & 1 vol. $\%$ & 2 vol. $\%$ \\
\hline \multicolumn{5}{|c|}{ Copper(II) Sulfate Concentration } \\
\hline $0.100 \mathrm{~N}$ & & $50 \mathrm{M}$ & $0.010 \mathrm{M}$ & $0.050 \mathrm{M}$ \\
\hline
\end{tabular}

An overall flowchart of experimental parameters is shown in Figure 4.14, with optimized parameters highlighted in blue. These parametric investigations were carried out to determine optimal conditions for desired goals of uniform deposition of copper nanoparticles and low copper concentration with minimum impurities. It was determined that the optimal conditions to achieve this goal are 3 minutes of activation time in 1 vol.\% of acetic acid, at a $\mathrm{CuSO}_{4}$ concentration of $0.010 \mathrm{M}$, using 0.33 moles of $\mathrm{Mg}$ ribbon. 


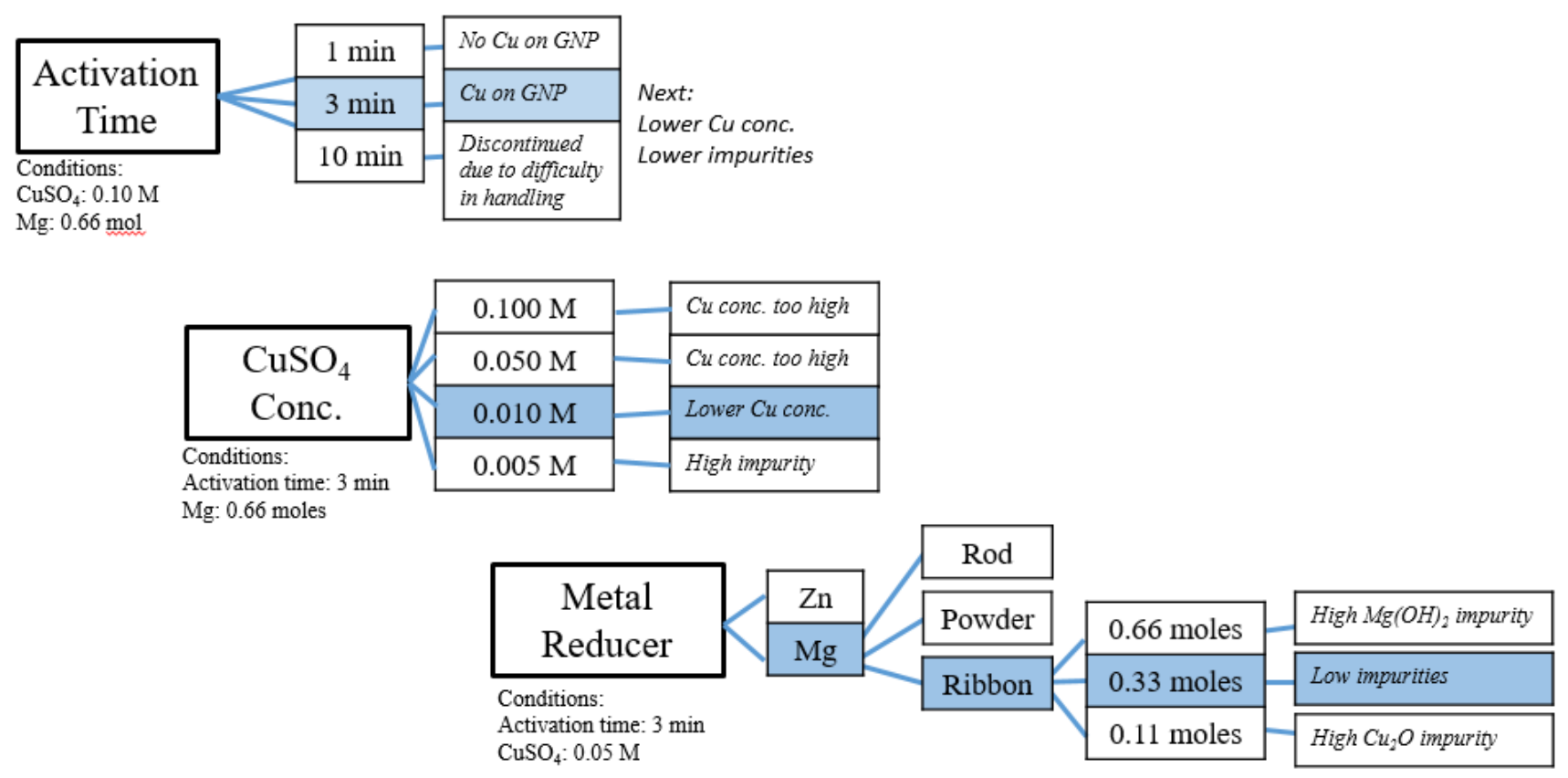

\begin{tabular}{|c|c|c|c|c|}
\hline \multirow{2}{*}{$\begin{array}{c}\text { Acetic Acid } \\
\text { Vol. } \%\end{array}$} & \multirow{2}{*}{$\begin{array}{l}1 \text { vol. } \% \\
2 \text { vol. } \% \\
\end{array}$} & \multirow{3}{*}{$\begin{array}{l}\text { Lower } \mathrm{Cu}_{2} \mathrm{O} \\
\text { impurity }\end{array}$} & \multicolumn{2}{|c|}{ with the following conditions } \\
\hline & & & Activation Time & $3 \min$ \\
\hline \multirow{4}{*}{$\begin{array}{l}\text { Conditions: } \\
\text { Activation time: } 3 \mathrm{~min} \\
\text { CuSO } 4.0 .05 \mathrm{M} \\
\text { Mg: } 0.33 \text { moles }\end{array}$} & & & Acetic Acid vol.\% & 1 vol. $\%$ \\
\hline & & & $\mathrm{CuSO}_{4}$ Conc. & $0.010 \mathrm{M}$ \\
\hline & & & Reducer & Ribbon \\
\hline & & & & 0.33 moles \\
\hline
\end{tabular}

Figure 4.14. Overall flowchart of experimental parameters. Blue color highlights the optimal conditions. 


\subsection{Preliminary Characterization of Sintered GNP-Cu Compacts}

Two GNP-Cu compacts were consolidated from the powder synthesized in order to determine the feasibility of obtaining a nacre like structure via spark plasma sintering (SPS) of GNP with uniform copper particle deposition. The conditions in which GNP-Cu powders were synthesized are detailed in Table 4.9. The conditions used for GNP-Cu-1 compact were $0.10 \mathrm{M} \mathrm{CuSO}_{4}, 2 \mathrm{vol} . \%$ acetic acid, and 0.66 moles of $\mathrm{Mg}$ ribbon. While the GNP-Cu-2 compact was synthesized with powder using the optimal conditions determined: $0.01 \mathrm{M} \mathrm{CuSO}_{4}, 1$ vol.\% acetic acid and 0.33 moles of $\mathrm{Mg}$ ribbon.

Table 4.9. GNP-Cu powder synthesis conditions detailing the concentrations of $\mathrm{Mg}$ ribbon and $\mathrm{CuSO}_{4}$, surface activation length, and volume \% of acetic acid used

\begin{tabular}{|c|c|}
\hline Powder for GNP-Cu-1 & Powder for GNP-Cu-2 \\
\hline \hline 0.66 moles of $\mathrm{Mg}$ ribbon & 0.33 moles of $\mathrm{Mg}$ ribbon \\
\hline $0.10 \mathrm{M} \mathrm{CuSO}{ }_{4}$ & $0.01 \mathrm{M} \mathrm{CuSO}_{4}$ \\
\hline 3 min surface activation & 3 min surface activation \\
\hline 2 vol.\% acetic acid & 1 vol.\% acetic acid \\
\hline
\end{tabular}

Spark plasma sintering parameters are listed in Table 4.10. The GNP-Cu-1 sample was sintered at the temperature of $1600{ }^{\circ} \mathrm{C}$ and pressure of $80 \mathrm{MPa}$. Since copper's melting point is $1085^{\circ} \mathrm{C}$, the high sintering temperatures and pressure used for this sample resulted in the vaporization of copper. This is unwanted since a slight melting of copper particles in order to obtain a nanometer scale metallic layer is desirable. Thus, the temperature was lowered for sample 2. The GNP-Cu-2 sample was sintered at the temperature of $850{ }^{\circ} \mathrm{C}$ and pressure of $80 \mathrm{MPa}$. Vaporization of copper was not observed at this temperature and pressure. 
Table 4.10. Temperature, pressure, heating rate and hold time conditions used to sinter GNP-Cu compacts via Spark Plasma Sintering

\begin{tabular}{|l|c|c|} 
& GNP-Cu-1 & GNP-Cu-2 \\
\hline \hline Temperature & $1600{ }^{\circ} \mathrm{C}$ & $850{ }^{\circ} \mathrm{C}$ \\
\hline Pressure & $80 \mathrm{MPa}$ & $80 \mathrm{MPa}$ \\
\hline Heating Rate & $200{ }^{\circ} \mathrm{C} / \mathrm{min}$ & $50{ }^{\circ} \mathrm{C} / \mathrm{min}$ \\
\hline Hold time & $5 \mathrm{~min}$ & $10 \mathrm{~min}$ \\
\hline
\end{tabular}

XRD patterns of both powders and compacts show the main peaks of GNP and copper (Figure 4.15). For GNP-Cu-1, peaks corresponding to magnesium impurities are observed in both the powder (as $\mathrm{Mg}(\mathrm{OH})_{2}$ ) and subsequently the compact (as $\mathrm{MgO}$ ). The higher temperatures present during sintering caused the $\mathrm{Mg}$ impurity to react as follows:

$$
\mathrm{Mg}(\mathrm{OH})_{2} \rightarrow \mathrm{MgO}+\mathrm{H}_{2} \mathrm{O}
$$

XRD analysis of the GNP-Cu-2 powder and compact does not show magnesium impurities. This is because the optimized concentration of $\mathrm{Mg}$ ribbon ( 0.33 moles) was used for this synthesis. 

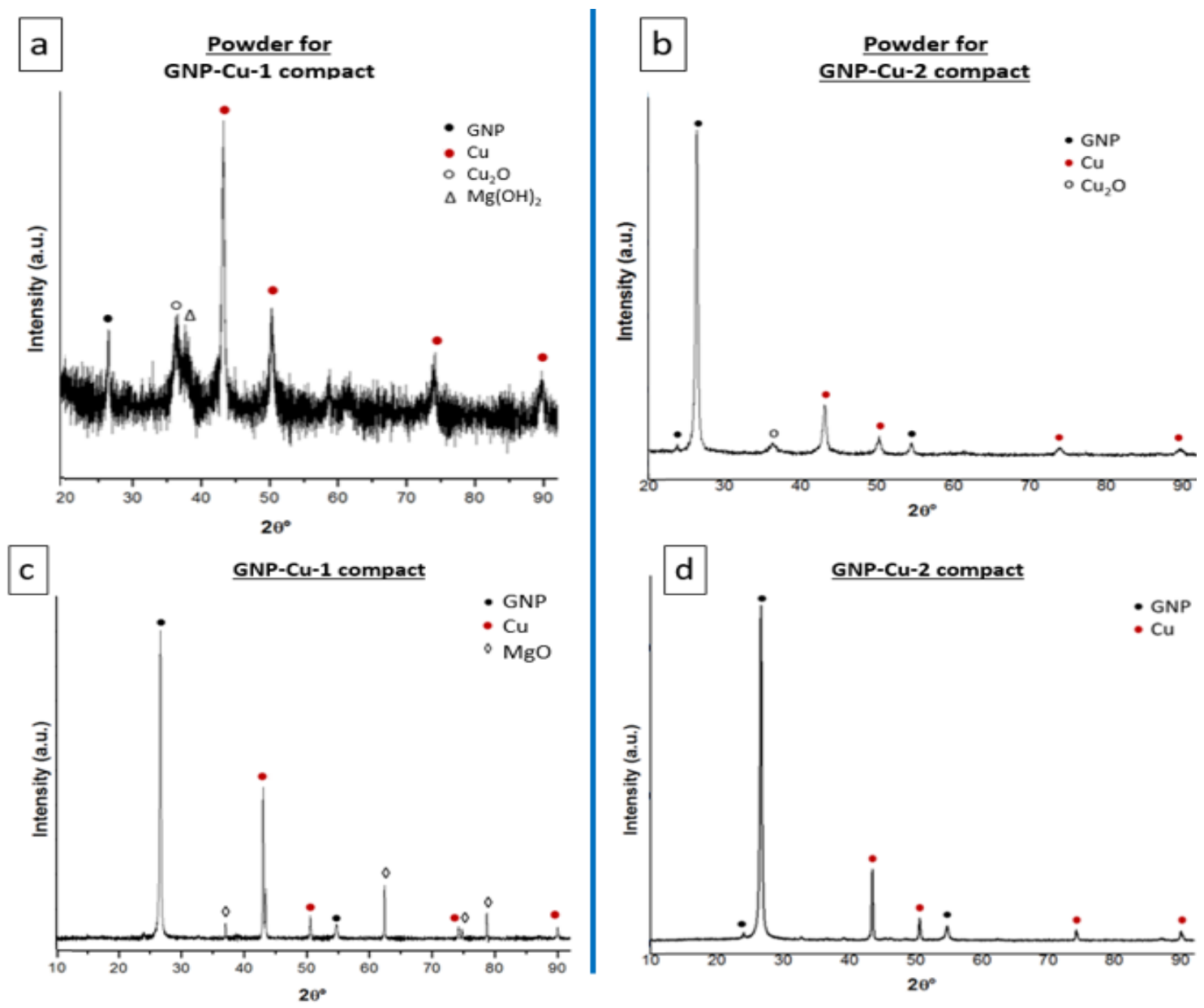

Figure 4.15. XRD patterns of pre (powder) and post (compact) SPS of GNP-Cu samples.

Density measurements of GNP-Cu-1 and GNP-Cu-2 powders and compacts are detailed in Table 4.14. From the true density values, the volume percentages of GNP and $\mathrm{Cu}$ in the powder and compacts were calculated and are also detailed in the same table. Lowering the concentration of $\mathrm{CuSO}_{4}$ used from $0.10 \mathrm{M}$ to $0.01 \mathrm{M}$ resulted in $\mathrm{GNP}-\mathrm{Cu}$ powder with a higher volume \% of GNP (from 86 v.\% to 91.5 v.\%) and lower volume \% of copper (from 14 v.\% to 9.5 v.\%). To best mimic nacre, obtaining 95 vol.\% GNP and 5 vol. $\% \mathrm{Cu}$ is desired. The porosity of the compacts was obtained via Archimedes principle. The percentage of GNP and $\mathrm{Cu}$ present in the sample was back calculated from the percent 
density of the compact. GNP-Cu-1 compact was calculated to be 72.2 v.\% GNP and 14.8 v. $\% \mathrm{Cu}$. This results in a ratio of $4.9 \mathrm{GNP}$ to $1 \mathrm{Cu}$. The GNP-Cu-2 compact was found to be 83.7 v.\% GNP and 9.3 v.\% $\mathrm{Cu}$, which is a ratio $9 \mathrm{GNP}$ to $1 \mathrm{Cu}$. Considering the ratio of GNP to $\mathrm{Cu}$ present in the compacts, the lower volume \% of uniformly dispersed $\mathrm{Cu}$ in the GNP-Cu-2 compact illustrates the effect that varying the concentration of $\mathrm{CuSO}_{4}$ ultimately has on the resulting samples.

Table 4.11. GNP-Cu powder and compact densities and the calculated volume percentages of GNP and $\mathrm{Cu}$

\begin{tabular}{|c|c|c|c|c|}
\hline & & $\begin{array}{c}\text { Pure GNP } \\
{[4]}\end{array}$ & GNP-Cu-1 & GNP-Cu-2 \\
\hline \multirow[t]{2}{*}{ Powder } & $\begin{array}{l}\text { Pycnometer } \\
\text { Density }\end{array}$ & $1.82 \mathrm{~g} / \mathrm{cm}^{3}$ & $2.81 \mathrm{~g} / \mathrm{cm}^{3}$ & $2.52 \mathrm{~g} / \mathrm{cm}^{3}$ \\
\hline & Volume \% & - & $\begin{array}{c}86 \text { vol.\% GNP } \\
14 \text { vol. } \% \mathrm{Cu}\end{array}$ & $\begin{array}{c}91.50 \text { vol. \% GNP } \\
9.50 \text { vol. } \% \mathrm{Cu}\end{array}$ \\
\hline \multirow[t]{4}{*}{ Compact } & $\begin{array}{c}\text { Pycnometer } \\
\text { Density }\end{array}$ & $2.11 \mathrm{~g} / \mathrm{cm}^{3}$ & $3.31 \mathrm{~g} / \mathrm{cm}^{3}$ & $2.79 \mathrm{~g} / \mathrm{cm}^{3}$ \\
\hline & $\begin{array}{l}\text { Archimedes } \\
\text { Density }\end{array}$ & - & $87 \%$ dense & $93 \%$ dense \\
\hline & $\begin{array}{l}\text { Volume \% } \\
\text { (considering } \\
\text { porosity) }\end{array}$ & - & $\begin{array}{c}72.20 \text { vol. } \% \text { GNP } \\
14.8 \text { vol. } \% \mathrm{Cu}\end{array}$ & $\begin{array}{c}83.70 \text { vol. } \% \text { GNP } \\
9.30 \text { vol. } \% \mathrm{Cu}\end{array}$ \\
\hline & Ratio of GNP:Cu & & $4.90: 1$ & $9: 1$ \\
\hline
\end{tabular}

\subsubsection{Fracture Surface of Compacts}

Fracture surface of GNP-Cu compacts shows the plausibility of creating a nacrelike structure via SPS of GNP-Cu powder synthesized in this work. Pure GNP powder and fracture surfaces of consolidated pure GNP, consolidated by Nieto et. al can be observed 
in Figure 4.16(a,b) [4]. GNP-Cu compacts were consolidated via SPS and SEM images of their starting powders and fracture surfaces are shown in Figure 4.16(c-h) below. The pure GNP and GNP-Cu compacts show dense layered structures, with stacked GNPs uniformly oriented perpendicular to the SPS pressing axis [4] (Figure 4.16b). Though the fracture surfaces of both GNP-Cu compacts also show stacked and uniformly oriented GNP layers, their surfaces greatly differ. Thick layers of copper are observed between the GNP layers in GNP-Cu-1 compact in Figures 4.16(d,e), while no thick metallic layers are observed in GNP-Cu- 2 compact, Figures 4.16(g,h). GNP-Cu- 1 compact was sintered at $1600^{\circ} \mathrm{C}$, which is above copper's melting point of $1085^{\circ} \mathrm{C}$ and at a high pressure of $80 \mathrm{MPa}$ resulting in vaporization of $\mathrm{Cu}$. The thick metallic layers observed are most likely due to melted copper clusters found in the GNP-Cu-1 powder as well as the higher copper concentration of this powder as well (Figure 4.16c). Due to the lower concentration and more effective dispersion techniques, copper clusters are not observed in as large of a frequency in GNP$\mathrm{Cu}-2$ powder (Figure 4.16f). This lower amount of $\mathrm{Cu}$ and its even deposition throughout the GNP surface results in the lack of thick metallic layer in GNP-Cu-2. Furthermore, the lack of thick metallic layer in GNP-Cu-2 compact indicates that the copper particles were deposited onto GNP in a more uniform manner and not clustered, resulting in nanometer scale copper layers. Most importantly, the fracture surface of GNP-Cu-2 compact shows signs of plastic deformation attributed to the presence of these nanometer scale copper layers found in between the GNPs (Figure 4.16g,h). 


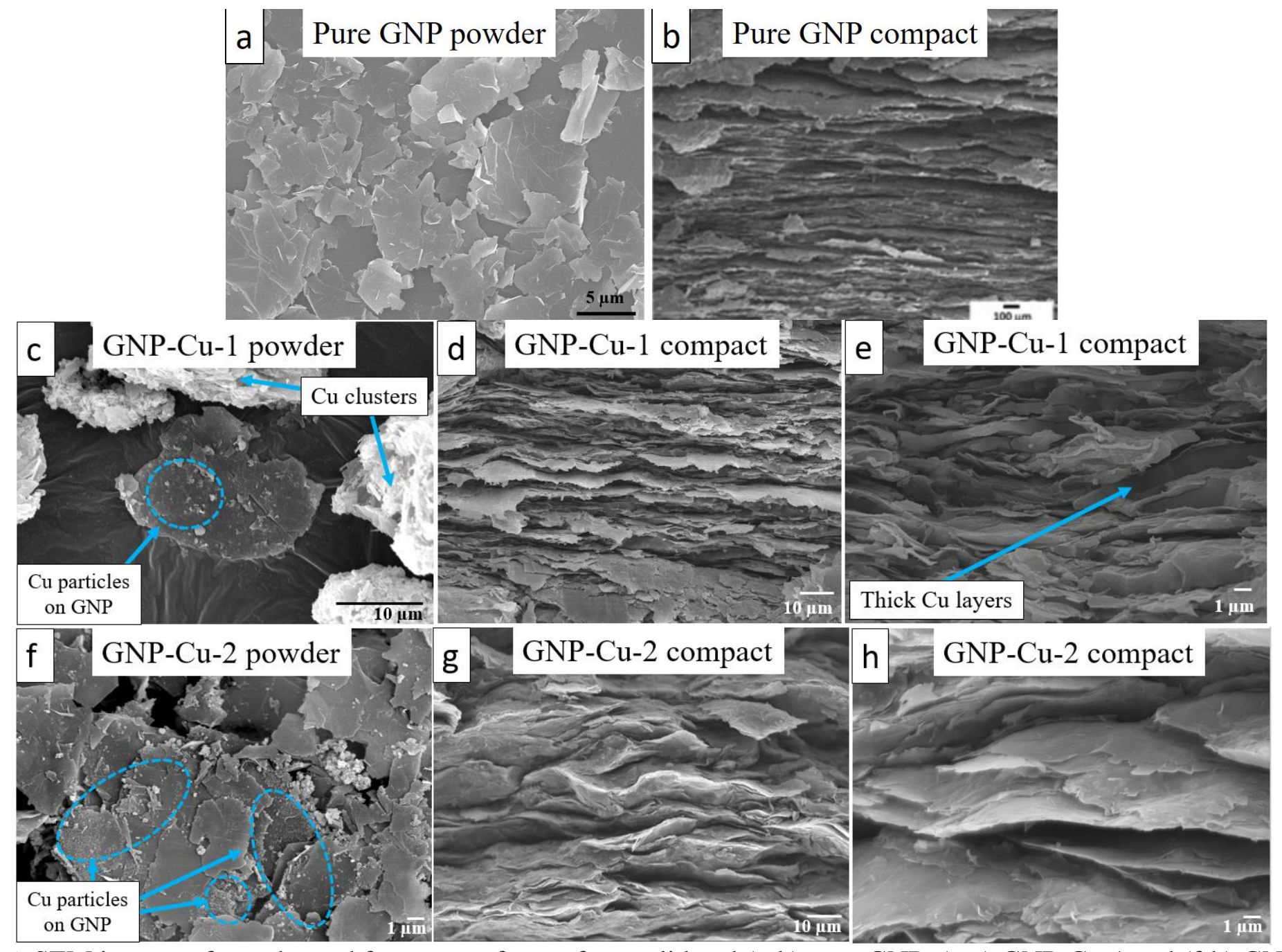

Figure 4.16. SEM images of powder and fracture surfaces of consolidated (a-b) pure GNP, (c-e) GNP-Cu-1 and (f-h) GNP-Cu-2. 
To further understand the uniformity of $\mathrm{Cu}$ distribution throughout the bulk compact, SEM images using the back-scattered detector were obtained. In SEM BackScattering Electron (BSE) mode, the signal is collected from beam collisions with the nuclei within the sample. Due to $\mathrm{Cu}$ having a larger atomic number compared to Carbon, it is expected that beam collision with $\mathrm{Cu}$ nuclei will occur more frequently, resulting in a "phase" contrast. Figure $4.17(\mathrm{a}, \mathrm{b})$ shows the fracture surface of GNP-Cu-2 as observed from secondary electron (SE) mode and BSE mode of SEM. Comparing both images allows one to see the relative $\mathrm{Cu}$ and $\mathrm{C}$ contrast, with the lighter colored areas corresponding to $\mathrm{Cu}$ and observed throughout the fracture surface of the compact.

Elemental mapping analysis of the GNP-Cu-2 fracture surface via EDS was also pursued in order to further understand the dispersion of $\mathrm{Cu}$ in the composite. The EDS elemental mapping shown in Figure 4.17 (c,d) shows that $\mathrm{Cu}$ is uniformly observed of throughout the fracture surface of the compact at varying magnifications. Though the aim of obtaining a GNP-Cu compact that is 95 vol.\% GNP and 5 vol.\% $\mathrm{Cu}$ has still not been achieved, the information collected from GNP-Cu-2 compact thus far is promising. 

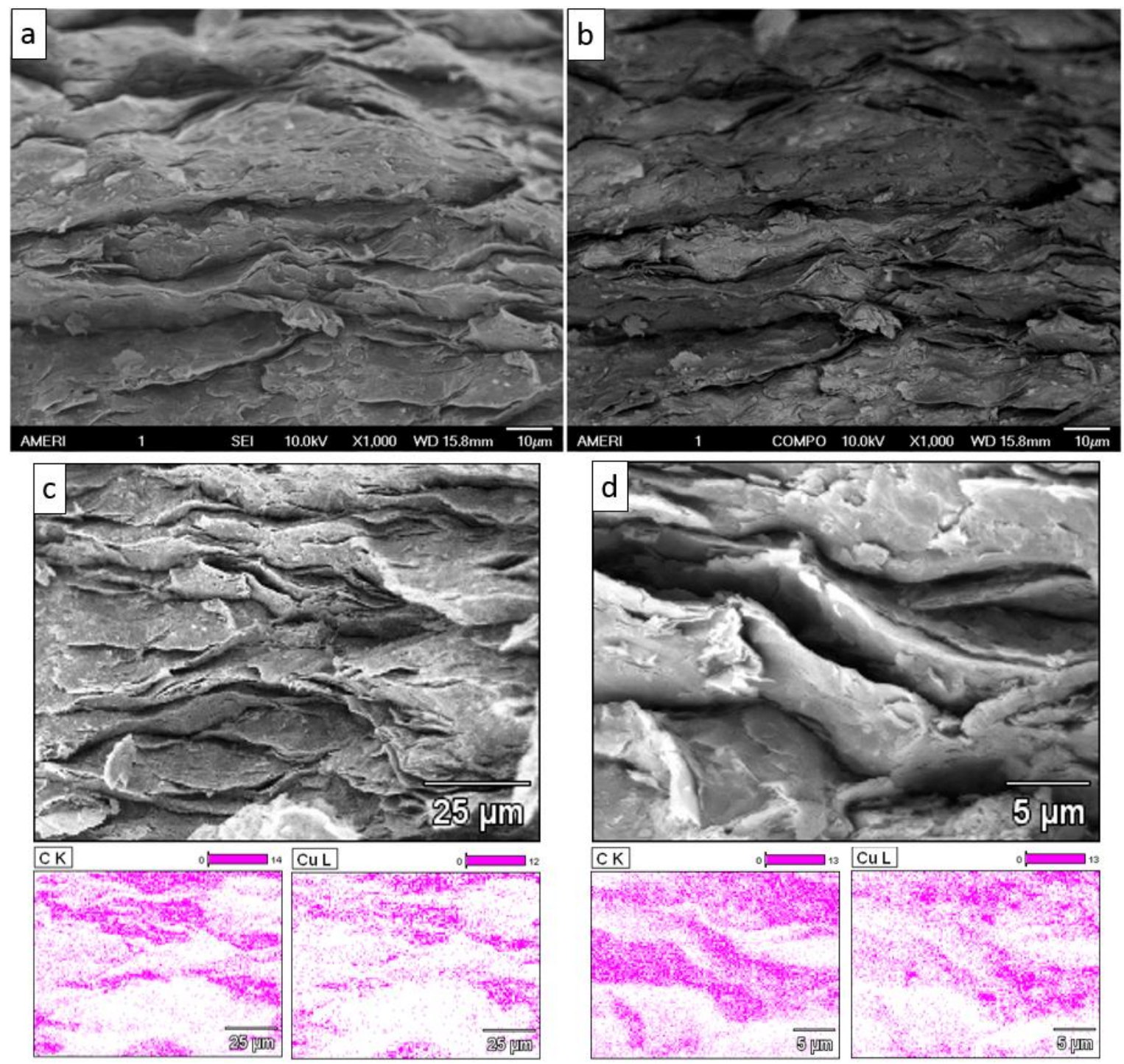

Figure 4.17. SEM images of the fracture surface of consolidated GNP-Cu-2 in SE (a) and BSE (b) modes, as well as EDS elemental mapping (c-d). 


\section{CHAPTER V}

\section{Conclusions}

In this thesis, copper particles were successfully displaced from copper(II) sulfate salt solution and deposited onto the surface of Graphene Nanoplatelets (GNP). Using the cementation process, the copper particles precipitated were in the nanometer scale (48.3$75.3 \mathrm{~nm}$ ) and successfully and evenly decorated the surface of GNP. This work also studied parameters in order to optimize the synthesis procedures. The GNP with copper particles (GNP-Cu) synthesized in this study were precursor materials to make a nacre-like structure through consolidation via SPS. Though the aim of this work is exclusively to synthesize GNP-Cu powder, preliminary investigation on sintered samples was also carried out as a feasibility study. The overall findings of this thesis are:

- $\mathrm{Mg}$ ribbon is the most effective form of $\mathrm{Mg}$ to act as a reducing agent for precipitating pure copper from $\mathrm{CuSO}_{4}$.

- The critical concentration of $\mathrm{Mg}$ ribbon reducer is 0.33 moles, higher concentration (0.66 moles) led to Mg impurities, while 0.11 moles led to greater copper(I) oxide formation.

- Copper particles in the nanometer scale of size $48.33 \mathrm{~nm}$ to $75.34 \mathrm{~nm}$ can be precipitated using cementation method varying $\mathrm{CuSO}_{4}$ solutions from $0.10 \mathrm{M}$ and $0.005 \mathrm{M}$. 
- 3 minutes is the optimal length of surface activation by acetic acid in order to obtain successful deposition of copper nanoparticles onto the surface of GNP.

- Lower $\mathrm{Cu}_{2} \mathrm{O}$ impurity was obtained when the volume percentage of acetic acid was decreased from 2 v. $\%$ to $1 \mathrm{v} . \%$.

- Fracture surface of GNP-Cu-2 compact shows signs of mimicking a nacre-like structure due to stacked GNP layers with nanometer scale copper layers amidst the GNP. 


\section{CHAPTER VI}

\section{Recommendations for Future Work}

The focus of this work was to uniformly deposit copper nanoparticles on the surface of Graphene Nanoplatelets (GNP) via a cementation reaction while considering parametric optimization. The findings of this work show that varying size of nanosized copper particles may be precipitated and deposited onto the surface of GNP by Mg ribbon. This resulting GNP-Cu powder may then be sintered to mimic a nacre-like structure. Based on the conclusions of this research, several recommendations for future work are made.

Synthesis and Parametric Studies:

- Further minimization of $\mathrm{Cu}$ precipitated must be done to obtain the desired volume percent ratio of 95 v.\% GNP and 5 v. $\% \mathrm{Cu}$.

- Carry out synthesis under vacuum conditions to lower copper oxide formation [41].

- Expand the findings of this thesis to deposit other metallic particles such as gold and silver onto GNP in for comparison studies. Due to the stable nature of these metals, it is anticipated lower oxide formation would be observed.

- The findings of this thesis may be expanded to deposit metallic particles on other 2D materials, such as Boron Nitride Nanoplatelets and MXenes.

\section{Characterization:}

- TEM analysis of GNP-Cu-2 compact should be carried out in order to best understand the size scale and uniformity of the copper layer amidst the GNP sheets that is not observed by SEM alone. 


\section{LIST OF REFERENCES}

[1] R. Mas-Balleste, C. Gomez-Navarro, J. Gomez-Herrero, F. Zamora. 2D materials: to graphene and beyong. Nanoscale 3 (2011) 20-30.

[2] W. Kim., S. Nair. Membranes from nanoporous 1D and 2D materials: A review of opportunities, developments and challenges. Chemical Engineering Science 104 (2013) 908-924.

[3] M. J. Allen, V. C. Tung, R. B. Kaner. Honeycomb Carbon: A Review of Graphene. Chemical Reviews 110 (2010) 132-145.

[4] A. Nieto, D. Lahiri, A. Agarwal. Synthesis and properties of bulk graphene nanoplatelets consolidated by spark plasma sintering. Carbon 50 (2012) 4068-4077.

[5] Chris Rudolf, B. Boesl, A. Agarwal. In situ indentation behavior of bulk multi-layer graphene flakes with respect to orientation. Carbon 94 (2015) 872-878.

[6] N. A. Kotov. Materials science: Carbon sheet solutions. Nature 442 (2006) 254-255.

[7] XG Sciences, "xGnP® Graphene Nanoplatelets Carbon Nanoparticles with Multifunctional Capability", XG Sciences Documentation (2009)

[8] N. Demirkiran, A. Kunkil. Recovering copper with metallic aluminum. Transactions of Nonferrous Metals Society of China 21 (2011) 2778-2782.

[9] A. G. Kulkarni, B. C. Pai, N. Balasubramanian. The cementation technique for coating carbon fibres. Journal of Materials Science 14 (1979) 592-598.

[10] B. C. Pai. Copper coating graphite particles. Materials Science and Engineering 21 (1975) 161-167.

[11] C. B. Lin, T. C. Wang, Z. C. Chang, H. Y. Chu. Tribological analysis of copper-coated graphite particle-reinforced A359 Al/5 wt.\% SiC composites. Journal of Materials Engineering and Performance 22 (2013) 94-103.

[12] F. Barthelat, D. Zhu. A novel biomimetic material duplicating the structure and mechanics of natural nacre. Journal of Materials Research 26 (2011) 1203-1215.

[13] M. T. Abba, P.M. Hunger, S. R. Kalidindi, U. G. K. Wegst. Nacre-like hybrid films: Structure, properties and the effect of relative humidity. Journal of the Mechanical Behavior of Biomedical Materials 55 (2016) 140-150. 
[14] H. Kakisawa, T. Sumitomo, R. Inoue, Y. Kagawa. The toughnening mechanism of nacre and structural materials inspired by nacre. Science and Technology of Advanced Materials 12 (2011) 064710.

[15] K. Lu. Sintering of nanoceramics. International Materials Reviews 53 (2008) 21-38.

[16] W. Choi, I. Lahiri, R. Seelaboyina, Y.S. Kang. Synthesis of Graphene and Its Applications: A Review. Critical Reviews in Solid State and Materials Sciences 35 (2010) 52-71.

[17] M. I. Katsnelson. Graphene: carbon in two dimensions. Materials Today 10 (2007) 20-27.

[18] K. W. Urban. Electron microscopy: The challenges of graphene. Nature Materials 10 (2011) 165-166.

[19] E. Stolyarova, K. T. Rim, S. Ryu, J. Maultzsch, P. Kim, L. E. Brus, T. F. Heinz, M. S. Hybertsen, G. W. Flynn. High-Resolution Scanning Tunneling Microscopy Imaging of Mesoscopic Graphene Sheets on an Insulating Surface. Proceedings of the National Academy of Sciences 104 (2007) 9209.

[20] B. Lang. A LEED study of the deposition of carbon on platinum crystal surfaces. Surface Science 53 (1975) 317-329.

[21] K. S. Novoselov, A. K. Geim, S. V. Morozov, D. Jiang, Y. Zhang, S. V. Dubonos, I. V. Grigorieva, A. A. Firsov. Electric field effect in atomically thin carbon films. Science 306 (2004) 666-669.

[22] M. Terrones, A.R. Botello-Mendez, J. Campos-Delgado, F. Lopez-Urias, Y. I. VegaCantu, F. J. Rodriguez-Macias, A. L. Elias, E. Munoz-Sandoval, A. G. Cano-Marquez, J.C. Charlier, H. Terrones. Graphene and graphite nanoribbons: Morphology, properties, synthesis, defects and applications. Nano Today 5 (2010) 351-372.

[23] V. Singh, D. Joung, L. Zhai, S. Das, S. I. Khondaker, S. Seal. Graphene based materials: Past, present and future. Progress in Materials Science 56 (2011) 1178-1271.

[24] C. Soldano, A. Mahmood, E. Dujardin. Production, properties and potential of graphene. Carbon 48 (2010) 2127-2150.

[25] V. Huc, N. Bendiab, N. Rosman, T. Ebbesen, C. Delacour, and V. Bouchiat. Large and flat graphene flakes produced by epoxy bonding and reverse exfoliation of highly oriented pyrolytic graphite. Nanotechnology 19, (2008) 455601.

[26]. A. Shukla, R. Kumar, J. Mazher, and A. Balan, Graphene made easy: high quality large-area samples. Solid State Communications 149 (2009) 718-721. 
[27] P. Blake, P. D. Brimicombe], R. R. Nair, T. J. Booth, D. Jiang, F. Schedin. Graphenebased liquid crystal device. Nano Letters 8 (2008) 1704-1708.

[28] Y. Hernandez, V. Nicolosi, M. Lotya, F. M. Blighe, Z. Sun, S. De. High-yield production of graphene by liquid-phase exfoliation of graphite. Nature Nanotechnology 3 (2008) 563-568.

[29] M. Lotya, Y. Hernandez, P. J. King, R. J. Smith, V. Nicolosi, L. S. Karlsson. Liquid phase production of graphene by exfoliation of graphite in surfactant/water solutions. Journal of American Chemical Society 131 (2009) 3611-3620.

[30] A. A. Green, M.C. Hersam. Solution phase production of graphene with controlled thickness via density differentiation. Nano Letters 9 (2009) 4031-4036.

[31] P. R. Somani, S. P. Somani, and M. Umeno. Planer nanographenes from camphor by CVD. Chemical Physics Letters 430 (2006) 56-59.

[32] A. N. Obraztsov, E. A. Obraztsova, A. V. Tyurnina, and A. A. Zolotukhin. Chemical vapor deposition of thin graphite films of nanometer thickness. Carbon 45 (2007) 20172021.

[33]. Q. Yu, J. Lian, S. Siriponglert, H. Li, Y. P. Chen, and S. S. Pei, Graphene segregated on Ni surfaces and transferred to insulators. Applied Physics Letters 93, (2008) 113103.

[34] K.S. Kim, Y. Zhao, H. Jan, S.Y. Lee, J.M. Kim, K.S. Kim. Large-scale pattern growth of graphene films for stretchable transparent electrodes. Nature 457 (2009) 706-710.

[35] A. Reina, X. Jia, J. Ho, D. Nezich, H. Son, V. Bulovic. Large area, few-layer graphene films on arbitrary substrates by chemical vapor deposition. Nano Letters 9 (2008) 30-35.

[36] Q. Yu, J. Lian, S. Siriponglert, H. Li, Y. P. Chen, and S. S. Pei. Graphene segregated on Ni surfaces and transferred to insulators. Applied Physics Letters 93 (2008) 113103.

[37] A. Malesevic, R. Vitchev, K. Schouteden, A. Volodin, L. Zhang, G.V. Tendeloo. Synthesis of few-layer graphene via microwave plasma-enhanced chemical vapour deposition. Nanotechnolgy 19 (2008) 305604.

[38] M. Zhu, J. Wang, B.C. Holoway, R.A. Outlaw, X. Zhao, K. Hou. A mechanism for carbon nanosheet formation. Carbon 45 (2007) 2229-2234.

[39] G. Eda, G. Fanchini, M. Chhowalla. Large-area ultrathin films of reduced graphene oxide as a transparent and flexible electronic material. Nature Nanotechnology 3 (2008) 270-274. 
[40] L. Jiao, L. Zhang, X. Wang, G. Diankov, H. Dai. Narrow graphene nanoribbons from carbon nanotubes. Nature 458 (2009) 877.

[41] T. T. Baby, S.S.J. Aravind, T. Arockiadoss, R.B. Rakshi, S. Ramaprabhu. Metal decorated graphene nanosheets as immobilization matrix for amperometric glucose biosensor. Sensors and Actuators B 145 (2010) 71-77.

[42] S. Stankovich, R. D. Piner, X. Chen, N. Wu, S. T. Nguyen, R.S. Ruoff. Stable aqueous dispersions of graphitic nanoplatelets via the reduction of exfoliated graphite oxide in the presence of poly(sodium 4-styrenesulfonate). Journal of Materials Chemistry 16 (2006) $155-158$.

[43] O. A. Fouad, S. Foda. Cementation-induced recovery of self-assembled ultrafine copper powders from spent etching solutions of printed circuit boards. Powder Technology 159 (2005) 127-134.

[44] Y. Li, W. Gao, L Ci, C. Wang, P. M. Ajayan. Catalytic performance of Pt nanoparticles on reduced graphene oxide for methanol electro-oxidation. Carbon 48 (2010) 1124-1130.

[45] K. S. Subramanyam, A. K. Manna, S. K. Pati, C.N.R. Rao. A study of graphene decorated with metal nanoparticles. Chemical Physics Letters 497 (2010) 70-75.

[46] H. Tien, Y. Huang, S. Yang, J. Wang, C. M. Ma. The production of graphene nanosheets decorated with silver nanoparticles for use in transparent, conductive films. Carbon 49 (2011) 1550-1560.

[47] Z. Xu, H. Gao, H. Guoxin. Solution-based synthesis and characterization of a silver nanoparticle-graphene hybrid film. Carbon 49 (2011) 4731-4738.

[48] Y. Ioni, E. Buslaeva, S. Gubin. Synthesis of graphene with noble metals nanoparticles on its surface. Materials Today: Proceedings 3S (2016) S209-S213.

[49] F. Wu, T. Huang, Y. Hu, X. Yang, Q. Xe. One-pot electrodeposition of a composite film of glucose oxidase, imidazolium alkoxysilane and chitosan on a reduced graphene oxide-Pt nanoparticle/Au electrode for biosensing. Journal of Electroanalytical Chemistry 781 (2016) 296-303.

[50] A. V. Kukhta, A. G. Paddubskaya, P. P. Kuzhir, S. A. Maksimenko, S. A. Vorobyova, S. Bistarelli, A. Cataldo, S. Bellucci. Copper nanoparticles decorated graphene nanoplatelets and composites with PEDOT:PSS. Synthetic Metals 222 (2016) 192-197.

[51] L. Lu, H. Li, F. Qu, X. Zhang, G. Shen. R. Yu. In situ synthesis of palladium nanoparticle-graphene nanohybrids and their application in nonenzymatic glucose biosensors. Biosensors and Bioelectronics 26 (2011) 3500-3504. 
[52] H. Di, Z. Yu, Y. Ma, F. Li, L. Lv, Y. Pan, Y. Lin, Y. Liu, Y. He. Graphene Oxide decorated with $\mathrm{Fe} 3 \mathrm{O} 4$ nanoparticles with advanced anticorrosive properties of epoxy coatings. Journal of the Taiwan Institute of Chemical Engineers 64 (2016) 244-251.

[53] H. S. Lim, J. W. Oh, S. Y. Kim, M. Yoo, S. Park, W. S. Lee. Anisotropically alignable magnetic boron nitride platelets decorated with iron oxide nanoparticles. Chemistry of Materials 25 (2013) 3315-3319.

[54] M. Taei, F. Hasanpour, M. Dinari, E. Dehghani. Au nanoparticles decorated reduced graphene oxide/layered double hydroxide modified glassy carbon as a sensitive sensor for electrocatalytic determination of phenazopyridine. Measurement 99 (2017) 90-97.

[55] L. Zhu, Y. Liu, P. Yang, B. Liu. Label-free aptasensor based on electrodeposition of gold nanoparticles on graphene and its application in the quantification of adenosine triphosphate. Electrochimica Acta 172 (2015) 88-93.

[56] X. Du, Z. Zhang, Z. Miao, M. Ma, Y. Zhang, C. Zhang, W. Wang, B. Han, Q. Chen. One step electrodeposition of dendritic gold nanostructures of $\beta$-lactoglobulinfunctionalized reduced graphene oxide for glucose sensing. Talanta 144 (2015) 823-829.

[57] X. Liu, J. Mao, P. Liu, X. Wei. Fabrication of metal-graphene hybrid materials by electroless deposition. Carbon 49 (2011) 477-483.

[58] H. F. Teoh, P. Dzung, W.Q. Lim, J.H. Chua, K. K. Lee, Z. Hu, H. Tan, E. S. Tok, C. $\mathrm{H}$. Sow. Microlandscaping on graphene oxide film via localized decoration of $\mathrm{Ag}$ nanoparticles. Nanoscale 6 (2014) 3143-3149.

[59] M. Karavasteva. Kinetics and deposit morphology of copper cementation onto zinc, iron and aluminum. Hydrometallurgy 76 (2005) 149-152.

[60] B. Donmez, F. Sevim, H. Sarac. A kinetic study of cementation of copper from sulphate solutions onto a rotating aluminum disc. Hydrometallurgy 53 (1999) 1450-154.

[61] T. Stefanowicz, M. Osinska, S. Napieralska-Zagozda. Copper recovery by the cementation method. Hydrometallurgy 47 (1997) 69-90.

[62] W. Lin, C. Wang, H. Le, B. Long, Y. Huang. Special assembly of laminated nanocomposite that mimics nacre. Materials Science and Engineering C 28 (2008) 10311037.

[63] B. Long, C. Wang, W. Lin, Y. Huang, J. Sun. Polyacrylamide-clay nacre-like nanocomposites prepared by electrophoretic deposition. Composites Science and Technology 67 (2007) 2770-2774. 
[64] K. Tushtev, M. Gonsior, M. Murck, G. Grathwohl, K. Rezwan. A novel bioinspired polymer-ceramic composite with outstanding crack resistance. Advanced Engineering Materials 16 (2014) 156-160.

[65] H. Kakisawa, T. Sumitomo, R. Inoue, Y. Kagawa. Fabrication of nature-inspired bulk laminar composites by a powder processing. Composites Science and Technology 70 (2010) 161-166.

[66] X. Zhang, C. Liu, W. Wu, J. Wang. Evaporation-induced self-assembly of organicinorganic ordered nanocomposite thin films that mimic nacre. Materials Letters 60 (2006) 2086-2089.

[67] X. Zeng, L. Ye, S. Yu, H. Li, R. Sun, J. Xu, C. P. Wong. Artificial nacre-like papers based on noncovalent functionalized boron nitride nanosheets with excellent mechanical and thermally conductive properties. Nanoscale 7 (2015) 6774-6781.

[68] J. L. He, W. Z. Li, H. D. Li, Simulation of nacre with TiN/Pt multilayers and a study of their hardness. Journal of Materials Research 12 (1997) 3140-3145.

[69] N. Almqvist, N. H. Thomson, B. L. Smith, G. D. Stucky, D. E. Morse, P. K. Hansma. Methods for fabricating and characterizing a new generation of biomimetic materials. Materials Science and Engineering C 7 (1999) 37-43.

[70] S. Deville, E. Saiz, R. K. Nalla, A. P. Tomsia. Freezing as a path of build complex composites. Science 311 (2006) 515-518.

[71] F. Bouville, E. Maire, S. Meille, B. Van de Moortele, A. J. Stevenson, S. Deville. Strong, tough, and stiff bioinspired ceramics from brittle constituents. Nature Materials 13 (2014) 508-514.

[72] C. M. Andres, N. A. Kotov. Inkjet deposition of layer-by-layer assembled films. Journal of American Chemical Society 132 (2010) 14496-14502.

[73] M. Mirkhalaf, F. Barthelat. Nacre-like materials using a simple doctor blading technique: fabrication, testing, and modeling. Journal of the mechanical behavior of biomedical materials 56 (2016) 23-33. 